\title{
Ricci-Flat Kähler Metrics on Affine Algebraic Manifolds and Degenerations of Kähler-Einstein K3 Surfaces
}

\author{
Ryoichi Kobayashi
}

\begin{abstract}
.
Some existence theorems of a complete Ricci-flat Kähler metrics on affine algebraic manifolds are established. As an application, we propose a picture describing the behavior of the Ricci-flat Kähler metrics under semi-stable degenerations of polarized $\mathrm{K} 3$ surfaces.
\end{abstract}

\section{$\S 0$. Introduction}

The theme of the present paper is the existence of a complete Ricciflat Kähler metric on certain class of affine algebraic manifolds. This paper is mostly of expository nature and is based on $[\mathrm{B}-\mathrm{K}]$, but has some original results on the existence of a complete Ricci-flat Kähler metric with moderate volume growth.

Affine algebraic manifolds which are expected to admit a complete Ricci-flat Kähler metric occur naturally in complex algebraic and differential geometry in many situations. First of all, we recall Yau's solution of Calabi's conjecture [Y2]:

Theorem ([Y2]). Let $M$ be a Kähler manifold. Suppose $\gamma$ is a real closed $(1,1)$-form which represents $2 \pi c_{1}(M)$. Then there exists a unique Kähler metric in each Kähler class such that its Ricci form is $\gamma$.

This theorem says that each admissible Kähler class contains a Kähler form which has a prescribed volume form. It opens a way to the investigation of complete Ricci-flat Kähler metrics. Namely we want to seek them as a singular perturbation of Kähler metrics with prescribed volume forms. Suppose that $D$ is a hypersurface in a compact complex

Received March 27, 1990.

Revised July 31, 1990. 
manifold $X$ such that $c_{1}(X)=\alpha[D]$ with $\alpha>0$. For instance, let $X$ be a compact complex manifold and $D$ a hypersurface. Clearly there exists a Ricci-flat volume form $V$ on $X-D$ with poles of appropriate order along $D$. Consider a one-parameter family of smooth volume forms $V_{t}$ on $X$ such that $\lim _{t \rightarrow \infty} V_{t}=V$. If $c_{1}(X)$ is positive, i.e., $X$ is a Fano manifold (e.g., $X=P_{n}(C)$ ), then there exists a unique Kähler metric $\omega_{t}$ such that $[\omega]$ is proportional to $c_{1}(X)$ and its volume form is $V_{t}$. We are interested in the limit as $t \rightarrow \infty$. We want to find conditions which guarantee the convergence of $\omega_{t}$ to a smooth Kähler metric on $X-D$. If the convergence turns out to be true, then we will reach a Ricci-flat Kähler metric on $X-D$ by a limiting process. We can easily construct such a family of Kähler metrics in the case of $X=P_{n}(C)$ and $D$ is a hyperplane. In this case, the limit metric is nothing but the standard flat metric of $C^{n}$. Although this approach seems to be natural, it has not yet been fully developed at this stage. Instead of following this line of argument, we will directly work on noncompact manifolds in Sections 2.3.1 and 2.3.3 to establish existence theorems. Only in Section 2.3.2, we will compare two approaches.

The well-known Eguchi-Hanson metric on the affine quadric surface is one of the first non-trivial examples of a complete Ricci-flat nonflat Kähler metric. Calabi [Ca1,2] generalized the Eguchi-Hanson metric to the ODE construction of Kähler-Einstein metrics on the total space of certain holomorphic vector bundles over Kähler-Einstein manifolds. Hitchin [Hit1] and Kronheimer [Kr] generalized Eguchi-Hanson metrics in other directions (twistor approach and hyperKähler quotients [Bes 2]) and clarified the close relationship between the ALE gravitational instantons and the Kleinian singularities. The boundary behavior of Eguchi-Hanson metric, complete Calabi metrics, Hitchin-Kronheimer metrics are called ALE boundary condition. See Nakajima's survey in this volume.

On the other hand, Hawking [Haw] and Gibbons-Hawking [GibHaw] discovered the Euclidean Taub-NUT metric on $R^{4}$ and its generalizations which turn out to be complete Ricci-flat Kähler metrics with anti-self-dual curvature form on $C^{2}$ and $A_{n}$-manifolds. These metrics are classified in the category of ALF gravitational instantons. The Riemannian geometry of the Taub-NUT metric differs from that of the standard flat metric in the following properties:

(i) the volume of geodesic ball grows like $r^{3}$,

(ii) the asymptotic behavior of the metric is described by rescaled Berger sphere metrics, i.e., the size of the Hopf fiber is asymptotically constant and the volume of the base $S^{2}$ grows like $r^{2}$, 
(iii) it interpolates the flat metrics of $R^{4}$ and $R^{3}$ by a one parameter family. We will discuss Taub-NUT type metrics in Section 2.2.4.

Ricci-flat Kähler metrics also appear as canonical metrics on the moduli spaces of stable vector bundles [I] and magnetic monopoles [AH]. Recently Nakajima (see Nakajima's survey in this volume) investigates the moduli space of Yang-Mills connections on ALE gravitational instantons and showed that the moduli space is again ALE if its dimension is four. Atiyah and Hitchin $[\mathrm{A}-\mathrm{H}]$ discovered that the moduli space of BPS monopoles of charge 2 with a canonical metric turns out to be an ALF gravitational instanton which differs from Gibbons-Hawking manifolds.

These examples do not cover the simplest case of $X-D$ where $X$ is a Fano manifold and $D$ is a smooth hypersurface such that $c_{1}(X)=\alpha[D]$. Yau announced in [Bou] that if $\alpha>1$ then there exists a complete Ricciflat Kähler metric on $X-D$. Bando and Kobayashi gave a simple proof for it under the assumption of the existence of a Kähler-Einstein metric on $D$. In Section 2.3.1 (see also 2.3.2) of Chapter 2, we review this proof in detail. In Section 2.3.3, we will consider the case $\alpha=1$. Since the boundary $D$ is a projective manifold with trivial canonical bundle, the Calabi-Yau theorem implies the existence of a Kähler-Einstein metric on $D$. So the Kähler-Einstein property at infinity is automatically fulfilled and the background metric is easily constructed. But, in this case, the volume of the metric balls grows like $r^{\frac{2 n}{n+1}}$ where $n$ is the complex dimension of the manifold. In surface case, the induced metrics of the metric spheres realize the collapsing $[\mathrm{Fu}]$ of almost flat metrics on the 3-dimensional nilmanifold to a 2-dimensional torus. In particular, the isoperimetric inequality does not hold for these metrics (also for ALF metrics and metrics approximating type III K3 surfaces). We overcome this difficulty by introducing weighted Sobolev inequalities (see Sections 2.2.3, 2.2.4 and 2.3.3).

This existence theorem in particular guarantees the existence of a complete Ricci-flat Kähler metric on affine cubic surfaces and more generally on type II degenerate K3 surfaces (and it is not difficult to modify the arguments in Section 2.3.3 to show the existence of a complete Ricci-flat Kähler metric on type III degenerate K3 surfaces also). These metrics on type II (resp. type III) degenerate K3 surfaces seem to be interesting objects in Riemannian geometry. The volume of geodesic ball grows like $r^{\frac{4}{3}}$ (resp. $r^{2}$ ). In the case of type II degenerate K3 surfaces, the family of induced metrics on geodesic spheres realizes the collapsing of almost flat metrics on a nilmanifold (e.g. a Heisenberg manifold). 
See Fukaya's survey in this volume. In the case of type III degenerate K3 surfaces, the asymptotic behavior of the metric is like that of the standard flat metric of $C^{*} \times C^{*}$.

In the surface case, we may consider some degenerate cases of $(X, D)$ with $\alpha=1$ in which $D$ is a degenerate curve. In fact, Gibbons-Hawking manifolds ([Gib-Haw] and [Hit2]), Atiyah-Hitchin manifolds ([A-H]), the Page-Hitchin manifold ([Pa] and [Hit2]) and $D_{n}$-manifolds (cf. [Pe]) are realized as a complement of an anti-canonical curve $D$ consisting of two smooth rational curves with one contact point. These manifolds with an ALF approximately Ricci-flat (in a similar sense as in Section 2.3.3) background metric are obtained from $C \times C^{*}$ or $C \times C^{*} /( \pm 1)$ by suitably modifying the boundary. Ricci-flat $D_{n}$-manifolds with ALF boundary condition is also known to Kronheimer (through twistor construction). If we consider a cycle of rational curves as a degenerate curve, we can cover type III degenerate K3 surfaces with a complete Ricci-flat Kähler metric of quadratic volume growth. The family of induced metrics on metric spheres realizes (after rescaling) the collapsing of solvmanifolds $([\mathrm{Fu}])$.

Using the special feature of real 4-dimension, it would be possible to describe the behavior of Ricci-flat Kähler metrics under semi-stable degenerations of polarized K3 surfaces. Here, the special feature in 4dimension is, roughly speaking, the "quantitative" conservation law of the Euler number (instanton number, which is $\frac{1}{8 \pi^{2}}$ times the squared $L^{2}$ norm of the curvature tensor). That is, the defect of the Euler number in the degeneration is captured as concentrated curvature of gravitational instantons which glue different components in the degeneration. In Section 2.3.3, although the mathematical footing is still vague, we propose a geometric picture describing this phenomenon in the spirit of implicit function theorem, i.e., approximating a $\mathrm{K} 3$ metric by gluing Ricci-flat Kähler metrics on affine algebraic manifolds. The gluing instantons turn out to be ALF gravitational instantons such as a Taub-NUT manifold in the case of Type II degenerations, and gravitational instantons with quadratic volume growth in the case of Type III degenerations. The latter instantons with quadratic volume growth are not known explicitly, but one can show a general existence theorem of these instantons (see [K3]). The boundary of these instantons are like that of $C^{*} \times C^{*}$ with a standard flat metric. So, the degenerate Kähler-Einstein K3 surface is, roughly speaking, composed of rational surfaces with a complete Ricciflat Kähler metric on the complement of the double curve (which is an anti-canonical divisor) and ALF gravitational instantons or "quadratic volume growth instantons" with concentrated curvature which glue ra- 
tional components. We note that this process approximates the CalabiYau metrics on K3 surfaces near the end (cusp) of the moduli space of polarized K3 surfaces.

For instance, a type III degenerate K3 surface of degree 4 consisting of the tetrahedron of $P_{2}(C)$ 's approximates Ricci-flat metrics on a K3 surface as those Ricci-flat metrics which look like four copies of $C^{*} \times C^{*}$ with a standard complete flat metric glued together by twenty four "quadratic volume growth instantons" of Euler number 1 with concentrated curvature. In the tetrahedral picture, four "quadratic volume growth instantons" lie on each edge. Outside of these Taub-NUT instantons, flat $C^{*} \times C^{*}$ 's are glued trivially by parts of flat $\left(C^{*} \times\right.$ cylinder $)$ and four flat solid tori corresponding to the four vertices. The conservation of the Euler number is clear in this picture. Namely, 24, the Euler number of a $\mathrm{K} 3$ surface, is the sum of $\frac{1}{8 \pi^{2}}$ times the squared $L^{2}$ norm of the curvature tensor of the twenty four "quadratic volume growth instantons".

Finally the author would like to say that the study of complete Ricciflat Kähler manifolds is only at a beginning stage. For instance, more general existence results should be established and the interactions with other Kähler-Einstein manifolds should be described. In particular, complete Ricci-flat Kähler metrics on the complement of an anti-canonical divisor of rational surfaces often appear as bubble-off instantons in the process of degeneration in which simple elliptic singularities occur. It is desirable to make a firm mathematical footing which explains such phenomena (cf. for the case of Kleinian singularities, see [B-K-N] and Nakajima's survey in this volume).

Acknowledgement. It is the author's pleasure to acknowledge here my intellectual debt to Shigetoshi Bando.

\section{Table of contents}

$\begin{array}{ll}0 . \text { Introduction } & 137\end{array}$

1. Some Kähler geometry 142

2. Ricci-flat Kähler metrics on affine algebraic manifolds $\quad 155$ $\begin{array}{ll}\text { 2.1. Calabi's construction } & 155\end{array}$

2.2. Calabi's construction on affine algebraic manifolds $\quad 158$ $\begin{array}{ll}2.2 .1 \text {. The case } \alpha>1 & 158\end{array}$

2.2.2. The case $\alpha=1 \quad 166$

2.2.3. Weighted Sobolev inequalities 172 
2.2.4. Weighted Sobolev inequalities and ALF gravitational instantons

2.3. Ricci-flat Kähler metrics on affine algebraic manifolds 191

$\begin{array}{ll}2.3 .1 \text {. The case } \alpha>1 & 191\end{array}$

2.3.2. Concentrating Ricci curvature at infinity 201

2.3.3. The case $\alpha=1$ and a geometric picture of degenerations of Kähler-Einstein metrics of K3 surfaces 204

\section{$\S 1$. Some Kähler geometry}

1. We get the category of complex manifolds if we assume that everything is holomorphic in the formal theory of smooth manifolds. For instance, the notion of holomorphic maps between complex manifolds is defined. $C^{n}$, open sets in $C^{n}$, manifolds defined by analytic equations, $P_{n}(C)$ (complex projective space), projective algebraic manifolds are typical examples of complex manifolds.

2. Let $X$ be a complex manifold and $X_{R}$ the underlying smooth manifold. There is a canonically defined $(1,1)$ tensor field $J$ with $J^{2}=$ -1 on $X_{R}$ called the complex structure tensor of $X$. Let $z_{\alpha}^{i}=x_{\alpha}^{i}+$ $\sqrt{-1} y_{\alpha}^{i}$ be a holomorphic local coordinate for $X$. Then $J$ is defined by

$$
\begin{aligned}
& J\left(\partial / \partial x_{\alpha}^{i}\right)=\partial / \partial y_{\alpha}^{i} \\
& J\left(\partial / \partial y_{\alpha}^{i}\right)=-\partial / \partial x_{\alpha}^{i}
\end{aligned}
$$

If we extend $J$ complex linearly to the complexified tangent bundle $T^{c}(X)=T\left(X_{R}\right) \otimes C$, then we have the splitting as complex vector bundles

$$
T^{c}(X)=T^{(1,0)}(X) \oplus T^{(0,1)}(X)
$$

into $\sqrt{-1}$ and $-\sqrt{-1}$ eigenspaces of $J$. The bundle $T^{(1,0)}(X)$ is the bundle of holomorphic vector fields and is locally spanned by holomorphic coordinate vector fields $\partial / \partial z_{\alpha}^{i}$.

3. Let $M$ be an $2 n$-dimensional smooth manifold. Suppose $M$ admits an almost complex structure, i.e., a $(1,1)$ tensor field $J$ with $J^{2}=-1$ on $M$. Define the $(2,1)$ tensor $N(J)$ by

$$
N(J)(X, Y)=[J X, J Y]-[X, Y]-J[X, J Y]-J[J X, Y] .
$$

Then the Newlander-Nirenberg Theorem states that there exists a unique complex manifold $X$ whose underlying smooth manifold is $M$ and whose complex structure tensor coincides with $J$. 
4. Let $X$ be a complex manifold and $J$ its complex structure tensor. A Riemannian metric $g$ on $X_{R}$ is a Hermitian metric if $J$ is an isometry with respect to $g$. We say that $(X, g)$ is a Hermitian manifold. For a Hermitian metric $g$, we define a 2 -form $\omega$ by

$$
\omega(X, Y)=g(X, J Y) \quad\left(X, Y \in \Gamma\left(T X_{R}\right)\right) .
$$

We call $\omega$ the Kähler form of $g$. If we extend $g$ complex linearly for complexified tangent vectors, we get a Hermitian metric $h$ for $T^{(1,0)} X$ which is a section of the complex vector bundle $T^{(1,0)}(X) \otimes \overline{T^{(1,0)}(X)}$. Let $\left.\left(z_{\alpha}^{i}\right)_{i=1}^{n}\right)$ be local holomorphic coordinates and set

$$
g_{\alpha, i \bar{j}}=h\left(\frac{\partial}{\partial z_{\alpha}^{i}}, \frac{\partial}{\partial z_{\alpha}^{j}}\right)=g\left(\frac{\partial}{\partial z_{\alpha}^{i}}, \frac{\partial}{\partial \overline{z_{\alpha}^{i}}}\right) .
$$

The Hermitian metric $h$ for $T^{(1,0)}(X)$ is written as

$$
h=\sum_{i=1}^{n} g_{\alpha, i j} d z_{\alpha}^{i} \otimes d \overline{z_{\alpha}^{j}} .
$$

Then the original Hermitian metric $g$ and the Kähler form $\omega$ are respectively the real part and the imaginary part of $h$ :

$$
\omega=\sqrt{-1} \sum_{i=1}^{n} g_{\alpha, i \bar{j}} d z_{\alpha}^{i} \wedge \overline{d z_{\alpha}^{j}} .
$$

It follows that if we fix the complex structure tensor $J$, then we may identify $g, h$ and $\omega$ via (1-4).

5. Since $\Lambda(V \oplus W)=\Lambda(V) \otimes \Lambda(W)$, the splitting (1-2) extends to exterior algebra bundle:

$$
\Lambda\left(T^{c}(X)\right)=\Lambda\left(T^{(1,0)}(X)\right) \otimes \Lambda\left(T^{(0,1)}(X)\right) .
$$

The right side is the union of bundles $\Lambda^{(p, q)}$ of $(p, q)$-forms which consist of $\mathrm{p} d z_{\alpha}^{i}$ 's and $\mathrm{q} d \overline{z_{\alpha}^{j}}$ 's. The exterior differentiation $d$ splits according to the splitting $(1-7)$ :

$$
d=\partial+\bar{\partial}: \Lambda^{(p, q)} \rightarrow \Lambda^{(p+1, q)} \oplus \Lambda^{(p, q+1)} .
$$

$d^{2}=0$ implies $\partial^{2}=0, \bar{\partial}^{2}=0$ and $\partial \bar{\partial}+\bar{\partial} \partial=0$. So we can consider the Dolbeault complex, etc. on a complex manifold.

6 . Let $E \rightarrow M$ be a smooth vector bundle of rank $r$ over a smooth manifold $X$. A connection $D$ for $E$ is a device for comparing vectors 
in $E$ with different base points in the sense that a connection defines differentiation of sections for $E$ along vector fields of $M$. More precisely, a connection $D$ for $E \rightarrow M$ is a linear map

$$
D: \Gamma(T M) \times \Gamma(E) \rightarrow \Gamma(E)
$$

assigning to $(X, \sigma)$ a new section $D_{X} \sigma$ such that for any smooth function $f$ on $M$,

$$
\begin{aligned}
D_{f X} \sigma & =f D_{X} \sigma \\
D_{X}(f \sigma) & =f D_{X} \sigma+(X f) \sigma .
\end{aligned}
$$

We say a section $\sigma$ is parallel if $D \sigma=0$. For any smooth curve segment $c:[0,1] \rightarrow M$ and a point $v$ in $E_{c(0)}$, we get a unique section $\tilde{c}$ such that $\tilde{c}(0)=v$ and $D_{\dot{c}} \tilde{c}=0$. This is the horizontal lift of $c$ through $v$. So, for any vector field $X$ of $M$ there is a unique horizontal lift $X_{H} \in \Gamma(T E)$. Let $M=\cup_{\alpha \in A} U_{\alpha}$ be an open covering of $M$ by such that for each $U_{\alpha}$ we have a local frame $e_{\alpha}=\left(e_{\alpha 1}, \cdots, e_{\alpha r}\right)$. Define a matrix valued 1-form $\left(\omega_{\alpha}\right)$ on $U_{\alpha}$ by

$$
D e_{\alpha}=e_{\alpha} \omega_{\alpha} \quad\left(D e_{\alpha i}=\sum_{j=1}^{r} e_{\alpha j} \omega_{\alpha i}^{j}\right)
$$

We say $\omega_{\alpha}=\left(\omega_{\alpha i}^{j}\right)$ the connection form for $D$ with respect to the frame $e_{\alpha}$. The Christoffel symbol is the components of the 1-form $\omega_{\alpha_{i}}^{j}$ with respect to local coordinates. The connection forms obey the following transition rules on overlaps $U_{\alpha} \cap U_{\beta}$ :

$$
\omega_{\beta}=g_{\alpha \beta}^{-1} \omega_{\alpha} g_{\alpha \beta}+g_{\alpha \beta}^{-1} d g_{\alpha \beta} .
$$

Conversely, if we are given $\omega_{\alpha}$ 's with the transition rules (1-10), we can define a unique connection $D$ by (1-9). Connections $D_{i}$ for $E_{i} \rightarrow M$ $(i=1,2)$ canonically induces connections for $E_{1} \oplus E_{2}, E_{1} \otimes E_{2}$ and the dual bundles $E_{i}^{*} \rightarrow M$. This is based on the derivation rule and the commutativity with the contraction. Let $h$ be a fiber metric for $E \rightarrow M$. Note that $h$ is a section of the bundle $E^{*} \otimes E^{*}\left(E^{*} \otimes \bar{E}^{*}\right)$ if $E$ is a real (complex) vector bundle. A connection $D$ is called a metric connection for $(E, h)$ if $D h=0$. The connection matrix of a metric connection satisfies the skew condition $\omega+{ }^{t} \omega=0$ for real bundles and $\omega+{ }^{t} \bar{\omega}=0$ for complex bundles with a Hermitian metric.

7. Let $E \rightarrow M$ and $D$ be as above. We use the covariant derivation $D$ on sections of $E$ and the exterior differentiation $d$ on differential forms 
to extend $D$ canonically to operate on differential forms with values in $D$. We thus get the exterior covariant differentiation $d^{D}$ such that, for any $\theta \in \Gamma\left(E \otimes \Lambda^{p}\right)$ and $\eta \in \Lambda^{q}$

$$
d^{D}(\theta \wedge \eta)=d^{D} \theta \wedge \eta+(-1)^{p} \theta \wedge d \eta
$$

It is easy to check that $R_{D}=d^{D} \circ D: \Gamma(E) \rightarrow \Gamma\left(E \otimes \Lambda^{2}\right)$ involves no differentiation, i.e., $R_{D}$ is an $\operatorname{End}(E)$-valued 2 -form. We call $R_{D}$ the curvature form of $D$. More generally we have

$$
d^{D} \circ d^{D}=R_{D} \wedge
$$

on the space of $E$-valued differential forms. Locally, $R_{D}$ is given by the system of matrix-valued 2 -forms

$$
\Omega_{\alpha}=d \omega_{\alpha}+\omega_{\alpha} \wedge \omega_{\alpha}
$$

with the transition rules

$$
\Omega_{\beta}=g_{\alpha \beta}^{-1} \Omega_{\alpha} g_{\alpha \beta}
$$

Since, for any vector field $X, Y$ on $M$ and an element $e \in E$, we have

$$
\left(\left[X^{H}, Y^{H}\right]-[X, Y]^{H}\right)_{e}=R_{D}(X, Y) e,
$$

the curvature vanishes if and only if the horizontal distribution in TE is locally integrable.

8. Let $E \rightarrow X$ be a holomorphic vector bundle of rank $r$ over a complex manifold $X$ and $h$ a Hermitian metric for $E \rightarrow X$. Let $D=D^{\prime}+D^{\prime \prime}$ be the decomposition of $D$ according to the decomposition (1-8). We say that $D$ is of type $(1,0)$ if $D^{\prime \prime}=\bar{\partial}$, i.e., the connection form is of type $(1,0)$ for a holomorphic frame.

Proposition 1. Let $(E, h)$ be as above. Then there exists a unique metric connection $D$ of type $(1,0)$, i.e., $D^{\prime \prime}=\bar{\partial}, D h=0$.

Proof. Let $e_{\alpha}$ be a local holomorphic frame. Applying $\partial$ to $h_{\alpha, i \bar{j}}=h\left(e_{\alpha i}, e_{\alpha j}\right)$ and using $D h=0$ and $D^{\prime \prime}=\bar{\partial}$, we have $\partial h_{\alpha, i \bar{j}}=$ $h\left(D^{\prime} e_{\alpha i}, e_{\alpha j}\right)$. This shows the uniqueness $\partial h_{\alpha, i \bar{j}}=h_{\alpha, k j} \omega_{\alpha i}^{k}$ for the connection form $\omega_{\alpha}$. It is easy to see that the 1 -forms $\omega_{\alpha}$ fulfill the transition rule $(10)$.

The connection in Proposition 1 is called the Hermitian connection for $(E, h)$.

9. For the curvature of a Hermitian connection, we have 
Proposition 2. Let $(E, h)$ be a holomorphic vector bundle with a Hermitian metric $h$ and $D$ its Hermitian connection. Then the curvature form $R_{D}$ is of type $(1,1)$ with values in the bundle of skew-Hermitian endomorphisms of $(E, h)$.

Proof. This follows from the two facts: (i) the curvature form is skew-Hermitian 2-form, i.e., $R_{D}=-{ }^{t} \overline{R_{D}}$ with respect to unitary frames, (ii) $d^{D^{\prime \prime}} D^{\prime \prime}=\bar{\partial}^{2}=0$. We get (i) by differentiating $h(e, e)=1$ twice where $e$ is a local section with norm 1 . We then have $R_{D}=d^{D} \circ D=$ $d^{D^{\prime \prime}} D^{\prime}+d^{D^{\prime}} D^{\prime \prime}$.

10. A Hermitian manifold $(X, g)$ is called a Kähler manifold if the Kähler form $\omega$ is a closed form: $d \omega=0$. The following proposition is well-known (see, for example, $[\mathrm{K}-\mathrm{N}]$ and [Gri-Har]):

Proposition 3. The following four conditions on a Hermitian manifold $(X, g)$ are equivalent:

(1) $(X, g)$ is a Kähler manifold,

(2) The complex structure tensor $J$ is parallel with respect to the LeviCivita connection of $g$,

(3) For each point $x \in X$, there are holomorphic local coordinates $\left(z^{i}\right)$ such that (i) $x^{i}(x)=0$ and (ii) $g_{i \bar{j}}(x)=\delta_{i j}, d g_{i \bar{j}}(x)=0$, i.e., $\omega=$ $\sqrt{-1} \sum_{i, j=1}^{n} \delta_{i j} d z^{i} \wedge d \overline{z^{j}}+O\left(|z|^{2}\right)$,

(4) The Kähler form $\omega$ is locally written as $\omega=\sqrt{-1} \partial \bar{\partial} f$ for some real valued function $f$,

(5) The Hermitian connection of the holomorphic tangent bundle coincides with the restriction of the Levi-Civita connection.

We call coordinates as in (3) holomorphic normal coordinates and a function $f$ in (4) a Kähler potential. For reader's convenience, we observe the meaning of (5). Let $e_{i}$ be a unitary frame and $\phi_{i}$ be its dual coframe. Let $\omega=\omega_{j i}$ be the connection matrix of the Hermitian connection for $\left(T^{(1,0)}(X), g\right)$. We then have

$$
D e_{i}=\sum_{j=1}^{n} e_{j} \omega_{j i}
$$

and the dual connection $D$ on $\left(T^{(1,0) *}(X), g\right)$ is determined by

$$
d(\omega(X))=(D \omega)(X)+\omega(D X)
$$


This implies that the connection matrix $\omega^{*}$ of the dual connection is determined by

$$
\omega^{*}=-{ }^{t} \omega .
$$

This equation and the following two relations are used to compute the connection matrix $\omega^{*}$ and hence $\omega$ :

$$
\begin{aligned}
\omega & =-{ }^{t} \bar{\omega} \\
\bar{\partial} \phi_{i} & =D^{\prime \prime} \phi_{i}=\sum_{j=1}^{n} \phi_{j} \otimes\left(\omega^{*}\right)_{j i}^{\prime \prime} .
\end{aligned}
$$

Hence we have

$$
d \phi_{i}=\sum_{j} \omega_{i j} \wedge \phi_{j}+\tau_{i}
$$

with $\tau_{i}$ a $(2,0)$-form. Since $\otimes$ has been replaced by $\wedge$, the $(2,0)$-form $\tau_{i}$ is the obstruction for the symmetry of the connection. In fact, if an affine connection is symmetric (i.e., $\Gamma_{j k}^{i}=\Gamma_{k j}^{i}$ ), the exterior differentiation is the composition of the covariant derivative and the anti-symmetrization operator. Thus the (1,2)-tensor determined by $\tau_{i}$ is the torsion tensor. The vanishing of the torsion tensor $\tau_{i}$ implies that the Hermitian connection coincides with the Levi-Civita connection. So the condition (5) is equivalent to the vanishing of the torsion tensor of the Hermitian connection.

11. (1) $C^{n}$ with the flat metric $\sqrt{-1} \partial \bar{\partial}\|Z\|^{2}$ is a complete Kähler manifold.

(2) $P_{n}(C)$ with the Fubini-Study metric

$$
\sqrt{-1} \partial \bar{\partial} \log \left(1+\|Z\|^{2}\right)
$$

is a compact Kähler manifold, where $Z=\left(z_{1}, \cdots, z_{n}\right)$ is the inhomogeneous coordinates. The Hopf fibration $S^{2 n+1} \rightarrow P_{n}(C)$ is a Riemannian submersion with respect to the standard metric of $S^{2 n+1}$ and (some constant multiple of) the Fubini-Study metric.

(3) $B^{n}$, the unit ball in $C^{n}$, with the Bergman metric $\sqrt{-1} \partial \bar{\partial} \log (1-$ $\left.\|Z\|^{2}\right)^{-1}$ is a complete Kähler manifold.

(4) A complex submanifold of a Kähler manifold is a Kähler manifold. In particular, any projective algebraic manifold is a compact Kähler manifold.

12. For the Levi-Civita connection and its curvature of a Kähler manifold $(X, g)$, we have the following Propositions (see $[\mathrm{K}-\mathrm{N}]$ and [GriHar]). 
Proposition 4. (1) All mixed components of the connection form with respect to local holomorphic coordinates vanish, i.e., all Christoffel symbols except $\Gamma_{j k}^{i}=\overline{\Gamma_{j \bar{j}}^{\bar{i}}}$ 's vanish. This implies that the Levi-Civita connection $\nabla$ restricts to the Hermitian connection of the holomorphic tangent bundle $T^{(1,0)}(X)$ with the Hermitian metric $g$.

(2) The curvature form of the complexified Levi-Civita connection splits into the $\operatorname{End}\left(T^{(1,0)}(X)\right)$-valued $(1,1)$-form and its complex conjugate. In particular, all remaining components of the curvature tensor are essentially of type $R_{\beta \gamma \bar{\delta}}^{\alpha}$.

If $R_{j k l}^{i}$ are the components of the curvature tensor of some Riemannian metric, then the Ricci curvature tensor is defined by the contraction

$$
R_{a b}=\sum_{i} R_{a i b}^{i}
$$

The following Proposition is very important in Kähler geometry because we can use cohomological and potential theoretic methods to study Kähler manifolds.

Proposition 5. If $\rho$ denotes the Ricci curvature tensor of the Riemannian metric $g$, then the Ricci-form $\gamma(X, Y)=\rho(X, J Y)$ is written as

$$
\gamma=-\sqrt{-1} \partial \bar{\partial} \log \operatorname{det}(g),
$$

where $\operatorname{det}(g)$ is the volume element with respect to local holomorphic coordinates. In particular, $\gamma$ is a real closed $(1,1)$-form and coincides with the trace of the curvature form.

13. It follows from (1) of Proposition 4 that, for $C^{2}$ functions $f$,

$$
\nabla_{i \bar{j}} f=\frac{\partial^{2} f}{\partial z^{i} \partial \bar{z}^{j}} .
$$

Therefore we have

$$
\triangle\left(\frac{1}{2} \text { of the Laplacian }\right)=\sum_{i, j=1}^{n} g^{i \bar{j}} \frac{\partial^{2}}{\partial z^{i} \partial \bar{z}^{j}} .
$$

Proposition 6. Let $(X, g)$ be a compact Kähler manifold of complex dimension n. A real closed $(1,1)$-form $\eta=\sqrt{-1} \sum a_{i \bar{j}} d z^{i} \wedge d \bar{z}^{j}$ is 
cohomologous to 0 in the de Rham cohomology if and only if there exists a real valued function $h$ such that $\eta=\sqrt{-1} \partial \bar{\partial} h$, i.e., $a_{i \bar{j}}=\frac{\partial^{2} h}{\partial z_{i} \partial z^{j}}$.

Proof. The "if" part is clear. We prove the "only" if part. Let $\omega$ be the Kähler form of $g$. Since $\eta$ is cohomologous to $0, \eta \wedge \frac{\omega^{n-1}}{(n-1) !}=$ $\left(\operatorname{tr}_{\omega} \eta\right) \wedge \frac{\omega^{n}}{n !}$ is also cohomologous to 0 . From the Hodge Theory, there exists a function $h$ such that $\triangle h=\operatorname{tr}_{\omega} \eta$. Setting $\tilde{\eta}=\sqrt{-1} \partial \bar{\partial} h$, we show $\tilde{\eta}=\eta$. Write $\tilde{\eta}=\sqrt{-1} \sum \tilde{a}_{i \bar{j}} d z^{i} \wedge d \overline{z^{j}}$ with $\tilde{a}_{i \bar{j}}=\partial^{2} h / \partial z^{i} \partial \overline{z^{j}}$. It follows from $\sum g^{i \bar{j}}\left(\tilde{a}_{i \bar{j}}-a_{i \bar{j}}\right)=0$ that

$$
\sum_{i, j} \nabla_{k}\left[g^{i \bar{j}}\left(\tilde{a}_{i \bar{j}}-a_{i \bar{j}}\right)\right]=\sum_{i, j} g^{i \bar{j}} \nabla_{k}\left(\widetilde{a}_{i \bar{j}}-a_{i \bar{j}}\right)=0 .
$$

Since $\widetilde{\eta}$ and $\eta$ are closed, we have

$$
\nabla_{k} a_{i j}=\nabla_{i} a_{k j}
$$

and the same equality with $\sim$. It follows from (1-17) and (1-18) that

$$
\bar{\partial}^{*}(\widetilde{\eta}-\eta)=\sum_{i} \sum_{k, j} g^{k \bar{j}} \nabla_{k}\left(\tilde{a}_{i \bar{j}}-a_{i \bar{j}}\right) d z^{i}=0
$$

Similarly, we have

$$
\partial^{*}(\tilde{\eta}-\eta)=0
$$

From (1-19) and (1-20), $\tilde{\eta}-\eta$ is a coclosed form. But since it is an exact form, the Hodge Decomposition Theorem implies that $\tilde{\eta}-\eta=0$.

Remark. Let $\eta=\sum \eta_{i j} d x^{i} \wedge d x^{j}$ be a 2 -form (considered as an alternative tensor field) on a Riemannian manifold. Then $d$ and its formal adjoint (with respect to the $L^{2}$ inner product) $d^{*}$ are locally written (up to constant multiple) as

$$
\begin{aligned}
(d \eta)_{i j k} & =\nabla_{i} \eta_{j k}-\nabla_{j} \eta_{i k}+\nabla_{k} \eta_{i j} \\
\left(d^{*} \eta\right)_{k} & =-g^{i j} \nabla_{i} \eta_{j k} .
\end{aligned}
$$

14. Let $E \rightarrow M$ be a complex vector bundle of rank $r$ over a compact smooth manifold $M$. Choose a connection $D$ and write $R_{D}$ for the curvature which is a 2 -form on $M$ with values in $\operatorname{End}(E)$. An invariant polynomial on $g l_{r}(C)$ is a polynomial function $P: g l_{r}(C) \rightarrow$ $C$ such that for all $X, Y \in g l_{r}(C), P(\operatorname{ad}(X) Y)=0$, in other words, 
$P\left(G X G^{-1}\right)=P(X)$ for all $G \in G L_{r}(C)$. The determinant and the trace are the invariant polynomials. Recall that the ring of invariant polynomials on $g l_{r}(C)$ is a polynomial ring generated by the polynomial functions

$$
c_{k}(X)=(2 \pi \sqrt{-1})^{-1} \operatorname{tr}\left(\Lambda^{k} X\right) .
$$

Indeed, it sufficed to check this for diagonal matrices. Therefore the elementary symmetric polynomials in the eigenvalues form a basis for the ring of invariant polynomials. But $\operatorname{tr}\left(\Lambda^{k} X\right)$ is the $k$-th elementary symmetric polynomial in the eigenvalues of $X$. Note that

$$
\operatorname{det}\left(1+t \frac{X}{2 \pi \sqrt{-1}}\right)=\sum_{k=0}^{r} t^{k} c_{k}(X)
$$

If we set $X=R_{D}$, then we get a globally defined (2k)-forms $c_{k}\left(R_{D}\right)$ on $M$ defined by (1-21).

Fact 1 (Chern-Weil correspondence). For any invariant polynomial $P$, the differential form $P\left(R_{D}\right)$ is closed and the de Rham cohomology class is independent of the choice of connection $D$ on $E$.

Proof. Let $D_{s}$ be a 1-parameter family of connections. The infinitesimal variation $a=\frac{d D_{s}}{d s}$ of connections is a 1 -form on $M$ with values in $\operatorname{End}(E)$. Then the infinitesimal variation of curvatures $\frac{d R_{D}}{d s}$ is $d^{D} a$. Therefore

$$
\begin{aligned}
\frac{d}{d s} \log \left(1+t R_{D}\right) & =t \operatorname{tr}\left\{\left(1+t R_{D}\right)^{-1} \frac{d R_{D}}{d s}\right\} \\
& =\sum_{k=0}^{\infty}(-1)^{k} t^{k+1} \operatorname{tr}\left(R_{D}^{k} d^{D} a\right)
\end{aligned}
$$

Now we use the Bianchi identity

$$
d^{D} R_{D}=0 .
$$

We then have from (24) that

$$
\operatorname{tr}\left(R_{D}^{k} d^{D} a\right)=\operatorname{tr}\left(d^{D}\left(R_{D}^{k} \wedge a\right)=d\left\{\operatorname{tr}\left(R_{D}^{k} \wedge d^{D} a\right)\right\},\right.
$$

which implies that the cohomology class of $\operatorname{det}\left(1+R_{D}\right)$ is independent of the choice of connections. Any connection is deformed locally to a 
flat trivial connection. This implies that $\operatorname{det}\left(1+t R_{D}\right)$ is locally exact, so it is closed.

The cohomology class of $c_{k}\left(R_{D}\right)$ is thus independent of the choice of connections and depend only on the vector bundle $E \rightarrow M$. We call $c_{k}\left(R_{D}\right)$ the $k$-th Chern form and the cohomology class $\left[c_{k}\left(R_{D}\right)\right]$ the $k$-th Chern class. It is known that the Chern classes are in fact defined in $H^{*}(M ; Z)$ (see [Hir], [M-S], [K-N] and [Gri-Har]). Although this differential geometric approach does not see torsion parts, it is still very useful. It follows from the definitions that the first Chern class of the bundle $E \rightarrow M$ coincides with the first Chern class of the determinant line bundle $\Lambda^{r}(E)$. If $E \rightarrow M$ is the holomorphic tangent bundle of a $n$-dimensional compact complex manifold $M$, then the Chern classes of $E \rightarrow M$ are called the Chern classes of $M$. The first Chern class of $M$ is the Chern class of the anti-canonical bundle $K_{M}^{-1}$, the line bundle of holomorphic $n$-vectors. Clearly the set of holomorphic line bundles forms a group $\operatorname{Pic}(M)$ (the Picard group) under the tensor product. The Chern class map

$$
c_{1}: \operatorname{Pic}(M) \rightarrow H^{2}(M ; Z)
$$

is a group homomorphism, i.e., $c_{1}(E \otimes F)=c_{1}(E)+c_{1}(F)$.

15. Let $L \rightarrow X$ be a holomorphic line bundle over a compact Kähler manifold $(X, g)$. Let $h=\left(h_{\alpha}\right)$ be a Hermitian metric on $L$ and $\sigma$ a holomorphic section of $L$. We choose the Hermitian connection of $(L, h)$. The curvature form

$$
\theta=-\sqrt{-1} \partial \bar{\partial} \log h_{\alpha}=-\sqrt{-1} \partial \bar{\partial} \log \|\sigma\|^{2}
$$

is $2 \pi$ times the Chern form. If we take a conformal change

$$
\|\cdot\|^{2} \rightarrow e^{-a}\|\cdot\|^{2}
$$

the curvature form changes as follows:

$$
\theta \rightarrow \tilde{\theta}=\theta+\sqrt{-1} \partial \bar{\partial} a .
$$

The following proposition is the converse of the above fact and is a direct consequence of Proposition 6.

Proposition 7. For any real closed real $(1,1)$-form $\tilde{\theta}$ in the cohomology class of $\theta$, there exists a Hermitian metric on $L$ with curvature form $\tilde{\theta}$. Such Hermitian metric is unique up to multiplication by positive constants. 
If $(X, g)$ is a compact Kähler manifold, then the Ricci form is the trace of the curvature form of the holomorphic tangent bundle with the Hermitian metric induced from the Kähler metric. So we have

Proposition 8. The Ricci form of a compact Kähler manifold is $2 \pi$ times the first Chern form of $X$.

16. Let $D$ be a closed hypersurface in an $n$-dimensional compact complex manifold $X$. We can cover $X=\cup U_{\alpha}$ by open sets so that if $D \cap U_{\alpha} \neq \emptyset$, there is a holomorphic function $f_{\alpha}$ on $U_{\alpha}$ with $D \cap U_{\alpha}=$ $\left\{f_{\alpha}=0\right\}$. We assign $f_{\alpha}=1$ to $U_{\alpha}$ if $U_{\alpha}$ does not meet $D$. We thus get a holomorphic line bundle $L_{D}$ defined by the transition functions $g_{\alpha \beta}=f_{\beta} / f_{\alpha}$ on $U_{\alpha} \cap U_{\beta} \neq \emptyset$. The line bundle $L_{D}$ does not depend on the various choices involved in its definition and is called the line bundle defined by $D$. The line bundle $L_{D}$ has a canonically defined holomorphic section $\sigma_{D}=\left(f_{\alpha}\right)$ with $\left(\sigma_{D}=0\right)=D$. If $D=\sum_{i=1}^{r} a_{i} D_{i}\left(a_{i} \in Z\right)$ is a divisor of $X$, i.e., a linear combination of closed hypersurfaces in $X$, we set

$$
L_{D}=\otimes_{i=1}^{r} L_{D_{i}}^{\otimes a_{i}} .
$$

Then clearly we have $c_{1}\left(L_{D}\right)=\sum_{i=1}^{r} a_{i} c_{1}\left(L_{D_{i}}\right)$. We write $[D] \in$ $H^{2}(X ; Z)$ for the Poincaré dual of the homology class in $H_{2 n-2}(X ; Z)$ defined by $D$.

Proposition 9. Let $D=\sum_{i=1}^{r} D_{i}$ be a divisor in a compact complex manifold $X$. Then we have

$$
c_{1}\left(L_{D}\right)=[D] .
$$

Proof. It suffices to prove this when $D$ is a hypersurface. Choose a Hermitian metric $h$ on $L$ and a section $\sigma_{D}$ with $\left(\sigma_{D}=0\right)=D$. Then we have $c_{1}\left(L_{D}, h\right)=\frac{1}{2 \pi \sqrt{-1}} \partial \bar{\partial} \log \|\sigma\|^{2}=-\frac{1}{2 \pi} d d^{c} \log \|\sigma\|^{2}$ where $d^{c}=\frac{\sqrt{-1}}{2}(\bar{\partial}-\partial)$. From the de Rham Theorem, it suffices to show

$$
\int_{X} c_{1}\left(L_{D}, h\right) \wedge \eta=\left.\int_{D} \eta\right|_{D}
$$

for arbitrary closed $(n-1, n-1)$-form on $X$. Let $U_{\alpha}$ be an open set in $X$ with holomorphic coordinates $\left(z_{\alpha}^{i}=z^{i}\right)$ such that $D \cap U_{\alpha}=\left\{z^{1}=0\right\}$. Let $X_{\varepsilon}=\{\|\sigma\| \geq \varepsilon\}$. Let $\pi: X-X_{\varepsilon} \rightarrow D$ be a smooth retraction defined on relatively compact domains in the regular part of $D$. In a 
neighborhood in $D \cap U_{\alpha}$, we can write

$$
\begin{aligned}
\eta & =\pi^{*}\left(\left.\eta\right|_{D}\right)+Q_{\alpha} \wedge d z^{1}+R_{\alpha} \wedge d \overline{z^{1}}+0\left(\left|z^{1}\right|\right) \\
d^{c} \log \|\left.\sigma\right|^{2} & =d^{c} \log h_{\alpha}+\operatorname{Im}\left(\frac{d z^{1}}{z^{1}}\right) .
\end{aligned}
$$

From the Stokes' formula, we have

$$
\begin{aligned}
\int_{X} c_{1}\left(L_{D}, h\right) \wedge \eta & =\lim _{\varepsilon \rightarrow 0} \int_{X_{\varepsilon}} \frac{1}{2 \pi} d^{c} \log \|\sigma\|^{2} \wedge \eta \\
& =\lim _{\varepsilon \rightarrow 0}\left[\left\{\left.\int_{X_{\varepsilon}} \frac{d \theta}{2 \pi} \wedge \pi^{*} \eta\right|_{D}\right\}+O(\varepsilon)\right] \\
& =\left.\int_{D} \eta\right|_{D}
\end{aligned}
$$

where $\theta$ stands for the argument of $z^{1}$. This completes the proof.

For example, the Chern class of the hyperplane bundle $L_{H}$ on $P_{n}(C)$ is the Poincare dual of the hyperplane $H$. The Chern class map gives the isomorphism

$$
\operatorname{Pic}\left(P_{n}(C)\right) \rightarrow H^{2}\left(P_{n}(C) ; Z\right) \cong Z
$$

and we have

$$
\begin{aligned}
K_{P_{N}(C)}^{-1} & =H^{n+1} \\
c_{1}\left(P_{n}(C)\right) & =(n+1)[H] .
\end{aligned}
$$

17. We shall use the following vanishing theorem later.

Proposition 10. Let $L \rightarrow X$ be a negative holomorphic line bundle with a Hermitian metric $h$ such that $\theta=-\sqrt{-1}$ (curvature form $)>0$ over an n-dimensional compact Kähler manifold with the Kähler metric $\theta$. Let $\triangle_{\theta}$ be the $\bar{\partial}$-Laplacian determined by the fiber metric $h$ and the Kähler metric $\theta$ acting on sections of $L$. If $\triangle_{\theta} u+\lambda u=0$ for $\lambda<n$, then $u \equiv 0$, i.e., any $\lambda<n$ is not an eigenvalue of $\triangle_{\theta}$. If the Ricci curvature of $\theta$ is nonnegative, then $n$ itself is not an eigenvalue of $\triangle_{\theta}$.

Proof. First let $(*): \triangle_{\theta} u+\lambda u=0$ for $\lambda<n$. Writing $\triangle$ for the 
Laplacian with respect to $\theta$ acting on functions, we have

$$
\begin{aligned}
0= & \int_{X}-\Delta h(u, u)=\int_{X} \operatorname{tr}_{\theta} \sqrt{-1} \bar{\partial} \partial h(u, u) \\
= & \int_{X} \operatorname{tr}_{\theta} \sqrt{-1} \bar{\partial}\left\{h\left(D^{\prime} u, u\right)+h\left(u, D^{\prime \prime} u\right)\right\} \\
= & \int_{X} \operatorname{tr}_{\theta} \sqrt{-1}\left\{h\left(d^{D^{\prime \prime}} D^{\prime} u, u\right)-h\left(D^{\prime} u, D^{\prime} u\right)\right. \\
& \left.\quad+h\left(D^{\prime \prime} u, D^{\prime \prime} u\right)+h\left(u, d^{D^{\prime}} D^{\prime \prime} u\right)\right\} \\
= & \int_{X} \operatorname{tr}_{\theta} \sqrt{-1} h\left(R_{D}(u), u\right)-\left\|D^{\prime} u\right\|_{L^{2}}^{2}-\left\|D^{\prime \prime} u\right\|_{L^{2}}^{2} \\
& \quad-\int_{X} \operatorname{tr}_{\theta} \sqrt{-1}\left\{h\left(d^{D^{\prime}} D^{\prime \prime} u, u\right)-h\left(u, d^{D^{\prime}} D^{\prime \prime} u\right)\right\} \\
= & (\lambda-n)\|u\|_{L^{2}}^{2}-\left\|D^{\prime} u\right\|_{L^{2}}^{2} \leq 0
\end{aligned}
$$

where we have used

$$
\begin{aligned}
\theta & =d^{D} D=d^{D^{\prime}} D^{\prime \prime}+d^{D^{\prime \prime}} D^{\prime} \\
\triangle_{\theta} & =-\bar{\partial}_{h}^{*} \bar{\partial}=-D^{\prime *} D^{\prime \prime}=\operatorname{tr}_{\theta} \sqrt{-1} d^{D^{\prime}} D^{\prime \prime} .
\end{aligned}
$$

So, if $\lambda<n$, then we have $u \equiv 0$. Note that $\lambda=n$ implies $D^{\prime} u \equiv$ 0 . Next, we assume $(*)$ with $\lambda=n$. Set $v=\bar{\partial} u$. Let $h^{\prime}$ be the Hermitian metric on the bundle $\Lambda^{(0,1)}(L)$ defined by $h$ and $\theta$. Integrating $-\triangle_{\theta} h^{\prime}(v, v)$ over $X$, we get

$$
\begin{aligned}
0= & \int_{X} \operatorname{tr}_{\theta} \sqrt{-1}\left\{h^{\prime}\left(R_{h^{\prime}}(v), v\right)-\left\|D^{\prime} v\right\|_{L^{2}}^{2}-\left\|D^{\prime \prime} v\right\|_{L^{2}}^{2}\right. \\
& +\int_{X} \operatorname{tr}_{\theta} \sqrt{-1}\left\{h^{\prime}\left(v, d^{D^{\prime}} D^{\prime \prime} v\right)-h^{\prime}\left(d^{D^{\prime}} D^{\prime \prime} v, v\right)\right\} \\
= & -\int_{X} h \otimes \operatorname{Ric}\left(v^{\sharp}\right)-\left\|D^{\prime} v\right\|_{L^{2}}^{2} .
\end{aligned}
$$

Therefore, if Ric $\geq 0$, then $D^{\prime} v \equiv 0$. So $D^{\prime}(\bar{\partial} u)=0$ and the exterior covariant differential $d^{D^{\prime}} D^{\prime \prime} u$ also vanishes. Combining this with $D^{\prime} u=$ 0 , we have $\theta(u)=0$. We thus have $u \equiv 0$.

18. Finally we prove the Chern-Lu formula for holomorphic maps between Kähler manifolds. For details we refer to [Ch2] and [Lu]. Let $M$ and and $N$ be a Kähler manifold and a Hermitian manifold. We equip $M$ and $N$ with the Hermitian connections. Let $f: M \rightarrow N$ 
be a holomorphic map. Set $u=\|\partial f\|^{2}$, where $\partial f \in \Gamma\left(T^{*(1,0)} M \otimes\right.$ $\left.f^{-1} T^{(1,0)} N\right)$. We write $\operatorname{Ric}_{M}$ and Bisect $_{N}$ for the Ricci curvature and the holomorphic bisectional curvature (of the Hermitian connections) of $M$ and $N$, respectively. The Chern-Lu formula is considered to be an infinitesimal Schwarz lemma.

Proposition 11 (Chern-Lu). Let $M, N$ and $u$ be as above. Then we have

$$
\triangle_{M} u=\operatorname{Ric}_{M}(\partial f, \overline{\partial f})-\operatorname{Bisect}_{N}(\partial f, \overline{\partial f}, \partial f, \overline{\partial f})+\left\|D^{\prime} \partial f\right\|^{2} .
$$

Proof. Since $f$ is holomorphic, we have $D^{\prime \prime} \partial f=0$. Therefore we have

$$
\begin{aligned}
\sqrt{-1} \partial \bar{\partial}(\partial f, \partial f)= & \sqrt{-1} \partial\left(\left(D^{\prime \prime} \partial f, \partial f\right)+\left(\partial f, D^{\prime} \partial f\right)\right) \\
= & \sqrt{-1}\left[\left(d^{D^{\prime}} D^{\prime \prime} \partial f, \partial f\right)-\left(D^{\prime \prime} \partial f, D^{\prime \prime} \partial f\right)\right. \\
& \left.\quad+\left(D^{\prime} \partial f, D^{\prime} \partial f\right)+\left(\partial f, d^{D^{\prime \prime}} D^{\prime} \partial f\right)\right] \\
& =(-\sqrt{-1} R(\partial f), \partial f)+\sqrt{-1}\left(D^{\prime} \partial f, D^{\prime} \partial f\right),
\end{aligned}
$$

because $d^{D^{\prime \prime}} d^{D^{\prime}}=R-d^{D^{\prime}} d^{D^{\prime \prime}}$. Taking the trace with respect to the Kähler form of $M$, we get

$$
\triangle_{M} u=-\operatorname{tr}_{M}(\sqrt{-1} R(\partial f), \partial f)+\left\|D^{\prime} \partial f\right\|^{2},
$$

where $R$ is the curvature form with respect to the Hermitian connection of the bundle $T^{*(1,0)} M \otimes f^{-1} T^{(1,0)} N$ with the metric induced from those of $M$ and $N$. Recall that it is of type $(1,1)$. Writing down this in terms of the curvatures of $M$ and $N$, we get the Chern-Lu formula.

\section{§2. Ricci-flat Kähler metrics on affine algebraic manifolds}

\subsection{Calabi's construction}

In this section we review Calabi's construction of Ricci-flat Kähler metrics on the total space of holomorphic line bundles. We refer to [C1] for more general constructions on holomorphic vector bundles. The basic idea is to use the Hermitian vector bundle structure over a KählerEinstein manifold to reduce the Kähler-Einstein condition, which is generally a Monge-Ampère equation, to an ordinary differential equation. Let $X$ be a compact complex manifold of dimension $n-1(n \geq 2)$ and $\pi: L \rightarrow X$ a holomorphic line bundle with a Hermitian metric $h$. Set

$$
\begin{aligned}
& t=-\log h(s, s) \quad(s \in L) \\
& \theta=\sqrt{-1} \partial \bar{\partial} t .
\end{aligned}
$$


We assume that $\left.\theta\right|_{X}=\theta_{X}$ is a Kähler-Einstein metric on $X$. Note that $\theta=\pi^{*} \theta_{X}$. Consider the following condition (D):

$$
\text { (D) : } c_{1}(X)=(\alpha-1) c_{1}(L), \quad(Q \ni \alpha \geq 1), \text { in } H^{2}(X ; Q) \text {. }
$$

In other words,

$$
\text { (D) : } c_{1}\left(K_{L}\right)=\alpha[X] \text { in } H_{\text {comp }}^{2}(L ; Q),
$$

where $[X]$ is the Poincaré dual of the 0 -section $X \subset L$ in $H_{\text {comp }}^{2}(L) \cong$ $H_{2 n-2}(L)$. Since $\theta_{X}$ is assumed to be a Kähler-Einstein metric, the condition (D) holds in the differential form level:

$$
\operatorname{Ric}\left(\theta_{X}\right)=(\alpha-1) \theta_{X}
$$

Hence, $(X, \theta)$ is a Kähler-Einstein manifold with positive Ricci curvature (if $\alpha>1$ ) or Ricci-flat (if $\alpha=1$ ). Kobayashi's Theorem [Ko1] implies that $X$ is simply connected if $\alpha>1$. We seek a Ricci-flat Kähler metric on $L$ in the form of $\sqrt{-1} \partial \bar{\partial} F(t)$. Set $f(t)=F^{\prime}(T)$. Then

$$
\omega_{0}=\sqrt{-1} \partial \bar{\partial} F(t)=f(t) \theta+f^{\prime}(t) \sqrt{-1} \partial t \wedge \bar{\partial} t .
$$

So

$$
\omega_{0}^{n}=(\sqrt{-1} \partial \bar{\partial} F(t))^{n}=f(t)^{n-1} f^{\prime}(t) \theta^{n-1} \wedge \sqrt{-1} \partial t \wedge \bar{\partial} t .
$$

If $\omega_{0}$ is a Kähler metric, then its Ricci-form is

$$
\operatorname{Ric}\left(\omega_{0}\right)=-\sqrt{-1} \partial \bar{\partial} \log \left(f(t)^{n}\right)^{\prime}+(\alpha-1) \theta .
$$

The Ricci-flat condition then becomes

$$
\log \left(f^{n}\right)^{\prime}=(\alpha-1) t+(\text { pluriharmonic function }) .
$$

If (pluriharmonic function) $\equiv$ constant, then we have

$$
f(t)=(A \exp \{(\alpha-1) t\}+B)^{\frac{1}{n}}
$$

if $\alpha>1$ and

$$
f(t)=(A t+B)^{\frac{1}{n}}
$$

if $\alpha=1$, where $A>0$ and $B$ are constants in both cases. It is easy to verify that $\omega_{0}$ is a Ricci-flat Kähler metric on the region $A e^{(\alpha-1) t}+B>0$ if $\alpha>1$, and on the region $\infty>A t+B>0$ if $\alpha=1$ both of which are 
complete toward the 0 -section $X$, i.e., $X$ lies at infinity. If $\alpha>1$, then $\omega_{0}$ is explicitly written as

$$
\omega_{0}=\left(A e^{(\alpha-1) t}+B\right)^{\frac{1}{n}}\left(\theta+\frac{\alpha-1}{n} \frac{A e^{(\alpha-1) t}}{A e^{(\alpha-1) t}+B} \sqrt{-1} \partial t \wedge \bar{\partial} t\right),
$$

and

$$
\omega_{0}^{n}=A e^{(\alpha-1) t}(\alpha-1) \theta^{n-1} \wedge \partial t \wedge \bar{\partial} t
$$

implies that $\omega_{0}^{n}$ is a singular Ricci-flat volume form on a neighborhood of $X$ in $L$ with poles of order $2 \alpha|d z|^{2} /|z|^{2 \alpha}$ along $X$. If $\alpha=1$, then $\omega_{0}$ is explicitly written as

$$
\omega_{0}=(A t+B)^{\frac{1}{n}}\left(\theta+\frac{A}{n(A t+B)} \sqrt{-1} \partial t \wedge \bar{\partial} t\right),
$$

and

$$
\omega_{0}^{n}=A \theta^{n-1} \wedge \sqrt{-1} \partial t \wedge \bar{\partial} t
$$

implies that $\omega_{0}^{n}$ is a singular Ricci-flat volume form on a neighborhood of $X$ in $L$ with logarithmic poles $|d z|^{2} /|z|^{2}$ along $X$. In both cases, the volume form $\omega_{0}^{n}$ is equal to $\eta \wedge \bar{\eta}$ where $\eta$ is a canonically defined (multivalued) holomorphic $n$-form on $L-X$ with poles of order $\alpha$ along $X$. Indeed, if $\alpha>1$, then $X$ is simply connected. This implies that the line bundle $L$ is obtained by covering and quotient operation from $K_{X}^{-1}$. Therefore all cases $\alpha>1$ are essentially the same because the condition $\alpha>1$ is preserved by covering and quotient operation. The case of $\alpha=2$ corresponds to $L=K_{X}^{-1}$. If $\alpha=2$, then $\omega_{0}$ with $B>0$ extends smoothly across the $\infty$-section of $L$ which is the 0 -section of $K_{X}$. Therefore the total space of the canonical bundle over a compact Kähler-Einstein Fano manifold admits a complete Ricci-flat Kähler metric and the 0 -section is totally geodesic. This can be seen in the following way. If $s^{\prime} \in K_{X}^{-1}$ and $h^{\prime}$ is the Hermitian metric of $K_{X}$ induced from that on $L=K_{X}^{-1}$, then we have in a neighborhood of the 0 -section $X \subset K_{X}$,

$$
\omega_{0}=\left(A h^{\prime}\left(s^{\prime}, s^{\prime}\right)+B\right)^{\frac{1}{n}}\left(\theta+\frac{1}{n} \frac{h^{\prime}\left(s^{\prime}, s^{\prime}\right)}{A h^{\prime}\left(s^{\prime}, s^{\prime}\right)+B} \sqrt{-1} \partial t \wedge \bar{\partial} t\right),
$$

where $t=\log h^{\prime}\left(s^{\prime}, s^{\prime}\right)$. This implies the extendability. From (33) we see that $B$ involved in $\omega_{0}$ parametrizes the volume of $\left(X, \omega_{0} \mid X\right)$. As $B \rightarrow 0, X$ is blown down to a singular point. We observe an example. The tautological line bundle $L=O_{P_{n-1}(C)}(-1)$ over $P_{n}(C)$ is $C^{n}$ with 
the origin blown up. Therefore the Ricci-flat Kähler metric on $L$ described above is the standard flat metric on $C^{n}$. The canonical bundle $K_{P_{n-1}(C)}=O_{P_{n-1}(C)}(1-n)$ is the quotient bundle of $L$ with respect to the action $z \rightarrow \exp \left(\frac{2 \pi \sqrt{-1}}{n}\right) z$ of the cyclic group $Z_{n}$. We have a family of complete Ricci-flat Kähler metrics $\omega_{0}$ with a fixed volume form on the canonical bundle parametrized by $B>0$. As $B \rightarrow 0$, the volume of the 0 -section goes to 0 and the curvature concentrates near the 0 -section. The limit $B=0$ corresponds to the flat orbifold metric of $C^{n} / Z_{n}$.

Remark. Let $X$ be a Fano Kähler-Einstein manifold of dimension $n-1$. Let $\omega$ be a complete a Ricci-flat Kähler metric with $B>0$ on the canonical bundle $K_{X}$. The following seems to be true (see [B-K-N] or Nakajima's survey in this volume): The $L^{n}$ norm of the full curvature tensor $\|R(\omega)\|_{L^{n}}$ is finite if and only if $(X, \omega)=\left(P_{n-1}(C)\right.$, the FubiniStudy metric).

\subsection{Calabi's construction on affine algebraic manifolds}

Throughout this section, we assume that $X$ is an $n$-dimensional Fano manifold, i.e., a compact complex manifold with ample anti-canonical bundle or equivalently, with positive first Chern class. Let $D$ be a smooth hypersurface in $X$ such that $c_{1}(X)=\alpha[D]$ with $\alpha>1$ or $\alpha=1$ in $H^{2}(X ; Q)$, where $[D]$ is the Poincaré dual of $D$. We apply Calabi's construction in a neighborhood of $D$ to get an approximately Ricci-flat complete Kähler metric on $X-D$.

\subsubsection{The case $\alpha>1$}

First of all, there is an upper bound for $\alpha \in Q$ in this case. Namely, we have $\alpha \leq n+1$. In fact, [H-K] and [K-O] showed that if $\alpha \geq n+1$, then $(X, D)$ is nothing but the hyperplane section $\left(P_{n}(C), P_{n-1}(C)\right)$. We have the standard flat metric in this case by applying Calabi's construction. From here on, we assume that $\alpha<n+1$.

Throughout this subsection, we assume that $D$ admits a KählerEinstein metric. The adjunction formula tells us that

$$
K_{D}=\left.\left(K_{X}+L_{D}\right)\right|_{D}
$$

and hence

$$
c_{1}(D)=\left.\left(c_{1}(X)-[D]\right)\right|_{D}=\left.(\alpha-1)[D]\right|_{D}>0 .
$$

Let $\sigma$ be a section of $L_{D}$ with $(\sigma=0)=D$. From the assumption, we have 
Lemma 1. The line bundle $[D] \rightarrow X$ admits a Hermitian metric $h$ such that the curvature form $\theta=-\sqrt{-1} \partial \bar{\partial} \log h(\sigma, \sigma)>0$ defines a Kähler metric on $X$ and the restriction $\left.\theta\right|_{D}$ of $\theta$ on $D$ is a KählerEinstein metric with positive Ricci curvature: $\operatorname{Ric}\left(\left.\theta\right|_{D}\right)=(\alpha-1) \theta_{D}$.

Following Calabi's construction, we set

$$
t=-\log h(\sigma, \sigma) .
$$

Then $D$ is the $\infty$-locus of $t$. Define

$$
\begin{aligned}
\omega_{0} & =\sqrt{-1} \partial \bar{\partial} \frac{n}{\alpha-1} \exp \left(\frac{\alpha-1}{n} t\right) \\
& =\exp \left(\frac{\alpha-1}{n} t\right)\left(\theta+\frac{\alpha-1}{n} \sqrt{-1} \partial t \wedge \bar{\partial} t\right) .
\end{aligned}
$$

From the assumption, $\omega_{0}$ is a complete Kähler metric on $X-D$. The metric asymptotically looks like Calabi's metric with $A=1$ and $B=0$ near $D$, i.e., at infinity. Let $\alpha=\frac{p}{q}$ with $0<p, q \in Z$. Since $L_{D}^{p}$ is a pluri-anti-canonical bundle $K_{X}^{-q}$, there exists a a $q$-ple holomorphic n-form $\eta_{\alpha}\left(d z_{\alpha}^{1} \wedge \cdots \wedge d z_{\alpha}^{n}\right)^{\otimes q}$ with poles of order $p$ along $D$. By taking $\left|\eta_{\alpha}\right|^{\frac{2}{q}}$, we get a canonical Ricci-flat volume form $V$ on $X-D$ with poles of order $2 \alpha=\frac{2 p}{q}$.

Lemma 2. Define a smooth function $f$ by $\omega_{0}^{n}=e^{f} V$. The function $f$ extends across $D$ to a smooth function on $X$ and the restriction of $f$ on $D$ is a constant function. So we may assume without loss of generality that $\left.f\right|_{D} \equiv 0$.

Proof. In this case, $\theta \neq\left.\pi^{*} \theta\right|_{D}$. This causes a trouble if $B \neq 0$. If $B \neq 0$ in $\omega_{0}$, then the differentiability of $f$ in the directions normal to $D$ no longer holds in general, because $A\|\sigma\|^{2}+B\|\sigma\|^{2 \alpha}$ appears in $\frac{\omega_{0}^{n}}{V}$. The term $\|\sigma\|^{2 \alpha}$ is not necessarily differentiable in the normal direction. If $B=0$ then $f$ extends across $D$ to a smooth function. Since

$$
\omega_{0}^{n}=e^{(\alpha-1) t} \theta^{n-1} \wedge \frac{\alpha-1}{n} \sqrt{-1} \partial t \wedge \bar{\partial} t(1+O(|z|)),
$$

where a smooth function in \{\} vanishes on $D$. So we compute

$$
\begin{aligned}
\sqrt{-1} \partial \bar{\partial} f_{D} & =\left.\sqrt{-1} \partial \bar{\partial}\left(\log \frac{\omega_{0}^{n}}{V}\right)\right|_{D} \\
& =\sqrt{-1}(\alpha-1) \partial \bar{\partial} t+\sqrt{-1} \partial \bar{\partial} \log \left(\left.\theta\right|_{D}\right)^{n-1} \\
& =\left.(\alpha-1) \theta\right|_{D}-\left.(\alpha-1) \theta\right|_{D}=0
\end{aligned}
$$


by the Einstein condition of $D$. This implies $\left.f\right|_{D} \equiv$ constant.

It follows from Lemma 2 that $f=O(\|\sigma\|)$. Let $a$ be a smooth function on $X$ which vanishes on $D$. We alter the Hermitian metric $h=\|\cdot\|^{2}$ to $h_{a}=e^{-a} h=e^{-a}\|\cdot\|_{a}$. Assume that the curvature form $\theta_{\boldsymbol{a}}$ of the new Hermitian metric $e^{-a} h$ is again positive. Then we get a new complete Kähler metric $\omega_{a}$ on $X-D$ by the same construction and a new function $f_{a}$ which also vanishes on $D$.

Lemma 3. There exists a $C^{\infty}$ function a on $X$ which vanishes on $D$ such that the new Hermitian metric $e^{-a} h$ on $L_{D}$ has the following properties:

(i) the curvature form $\theta_{a}$ is positive everywhere on $X$,

(ii) the function $f_{a}$ satisfies $f_{a}=O\left(\|\sigma\|_{a}^{2}\right)$.

Proof. It suffices to choose $a$ so that

$$
\left.\frac{\partial f_{a}}{\partial \sigma}\right|_{\sigma=0}=0
$$

because this implies $f_{a}=O\left(\|\sigma\|_{a}^{2}\right)$. We mean by $\left.\frac{\partial f}{\partial \sigma}\right|_{\sigma=0}$ a smooth section of $\left.L_{D}^{-1}\right|_{D}$ determined locally by $\left.\frac{\partial f}{\partial \sigma_{i}}\right|_{\sigma_{i}=0}$, where $\left\{\sigma_{i}=0\right\}$ are local defining equations of $D$. Indeed, this system of smooth functions defined locally on $D$ forms a smooth section of the bundle with transition function $g_{i j}=\frac{\sigma_{j}}{\sigma_{i}}$. We choose a local holomorphic frame for $L_{D}$ such that at $p \in D$ the connection form of the Hermitian connection vanishes; $\partial \log h_{i}(p)=0$. The following computation is done at $p \in D$.

$$
\begin{aligned}
\frac{\partial f_{a}}{\partial \sigma_{i}}= & \frac{\partial}{\partial \sigma_{i}}\left(\log \frac{\omega_{a}^{n}}{e^{-f} \omega_{0}^{n}}\right) \\
= & \frac{\partial}{\partial \sigma_{i}} \log e^{(\alpha-1) a+f} . \\
& \frac{(\theta+\sqrt{-1} \partial \bar{\partial} a)^{n-1} \wedge(\partial(t+a)) \wedge \overline{(\partial(t+a))}\left(1+O\left(\left|\sigma_{i}\right|^{2}\right)\right.}{\theta^{n-1} \wedge \partial t \wedge \bar{\partial} t\left(1+O\left(\left|\sigma_{i}\right|^{2}\right)\right.} \\
= & (\alpha-1) \frac{\partial a}{\partial \sigma_{i}}+\frac{\partial f}{\partial \sigma_{i}} \\
+ & \frac{\partial}{\partial \sigma_{i}} \log \frac{\theta_{0}^{n-1}\left(1+\triangle_{\theta_{0}} a+\cdots\right) \wedge\left|\frac{d \sigma_{i}}{\sigma_{i}}\right|^{2}\left(1+O\left(\left|\sigma_{i}\right|\right)-\sigma_{i} \frac{\partial a}{\partial \sigma_{i}}+\cdots\right)}{\theta_{0}^{n-1} \wedge\left|\frac{d \sigma_{i}}{\sigma_{i}}\right|^{2}\left(1+O\left(\left|\sigma_{i}\right|\right)\right)} \\
= & \triangle_{\theta_{0}} \frac{\partial a}{\partial \sigma_{i}}+(\alpha-2) \frac{\partial a}{\partial \sigma_{i}}+\frac{\partial f}{\partial \sigma_{i}},
\end{aligned}
$$


where if $\left(z_{i}^{1}, \cdots, z_{i}^{n-1}, \sigma_{i}\right)$ are local coordinates around $D$, then $\theta_{0}$ is the part of $\theta$ involving only $d z_{i}$ 's and $d \bar{z}_{i}$ 's, the symbol $O\left(\left|\sigma_{i}\right|\right)$ expresses the same thing in both numerator and denominator, and $+\cdots$ consists of terms with no contribution in the computation of $\partial / \partial \sigma_{i}$. The Laplacian $\triangle_{\theta_{0}}$ stands for the $\bar{\partial}$-Laplacian on $L_{D}^{-1}$ determined by the Hermitian metric $h$ and the Kähler metric $\left.\theta\right|_{D}$. We want to solve the equation

$$
\triangle_{\theta_{0}} s+(\alpha-2) s+\frac{\partial f}{\partial \sigma_{i}}=0 .
$$

We can apply Proposition 10 by setting $X=D$ and $L=L_{D}^{-1}$. We infer that any $\lambda<n-1$ is not an eigenvalue of $\triangle_{\theta_{0}}$. Hence the operator $\triangle_{\theta_{0}}+(\alpha-2)$ is injective for $\alpha \leq n$. Since the operator is self-adjoint, it is also surjective. So the equation (2-13) has a unique solution $s$. After multiplying a large constant to $\left\{\sigma_{i}\right\}$ if necessary, we can easily find a smooth function $a$ on $X$ such that $\left.a\right|_{D} \equiv 0, \frac{\partial a}{\partial \sigma_{i}}=s$ and such that $e^{-a} h$ has positive curvature.

From here on, we assume that the Hermitian metric $h$ fulfills the conditions in Lemma 3.

We proceed to study the asymptotic properties of the complete Kähler manifold $\left(X-D, \omega_{0}\right)$. Our situation is summarized as follows: (i) a pair $(X, D)$ of a Fano manifold $X$ and a smooth hypersurface $D$ with $c_{1}(X)=[D]$,

(ii) $\sigma \in H^{0}\left(X, O_{X}(D)\right)$ with $(\sigma=0)=D$ and a Hermitian metric $h$ on $L_{D}$ such that if we set $t=-\log h(\sigma, \sigma)$, then $\theta=\sqrt{-1} \partial \bar{\partial} t>0$ and $\left.\theta\right|_{D}$ is a Ricci-flat Kähler metric on $D$,

(iii) $\omega_{0}=e^{\frac{\alpha-1}{n}}\left(\theta+\frac{\alpha-1}{n} \sqrt{-1} \partial t \wedge \bar{\partial} t\right)$ is a complete Kähler metric on $X-D$ which is asymptotically Ricci-flat in the sense that $f=\log \frac{\omega_{0}^{n}}{V}=$ $O(h(\sigma, \sigma))$.

From the properties (i), $\cdots,($ iii), we have the following Lemma 4 . Let $d=d(*)=\operatorname{dist}(p, *)$ denote the distance function from a fixed point $o \in X-D, B(d)$ the metric ball of radius $\rho$ centered at $o$ and $S(d)$ the metric sphere $\partial B(d)$. Set $\rho=e^{\frac{(\alpha-1) t}{2 n}}=\|\sigma\|^{-\frac{\alpha-1}{n}}$.

Lemma 4. There exists a positive constant $c$ such that

(1) $c(d+1)<\rho<c^{-1}(d+1)$,

(2) $c d^{2 n}<\operatorname{Vol}(B(d))<c^{-1} d^{2 n}$

(3) $c d^{2 n-1}<\operatorname{Area}(S(d))<c^{-1} d^{2 n-1}$.

Proof. Let $p$ be a point in $X-D$ with $\|\sigma\|=\varepsilon$ which is sufficiently 
small with respect to $\|\sigma\|(o)$. It follows from (2-11) that

$$
\begin{aligned}
d & =\operatorname{dist}(o, p) \approx \int_{\varepsilon} \frac{d r}{r^{\frac{\alpha-1}{n}}+1} \approx \varepsilon^{-\frac{\alpha-1}{n}} \\
& =\|\sigma\|(p)^{-\frac{\alpha-1}{n}}=e^{\frac{(\alpha-1) t}{2 n}}=\rho .
\end{aligned}
$$

and that asymptotically

$$
\operatorname{Vol}(B(o, d)) \approx \int_{\varepsilon} \frac{r d r}{r^{2 \alpha}} \approx\|\sigma\|^{-2(\alpha-1)} \approx \rho^{2 n} \approx d^{2 n} .
$$

and

$$
\operatorname{Area}(S(o, d)) \approx\left(e^{\frac{(\alpha-1) t}{n}}\right)^{\frac{1}{2}+n-1} \approx \rho^{2 n-1} \approx d^{2 n-1}
$$

Definition. An $m$-dimensional complete Riemannian manifold $(M, g)$ is of $C^{k, \alpha}$-asymptotically flat geometry for $k \in N$ and $0<\alpha<1$ if for any $p \in M$ with $d(o, p)=r$ there exists a coordinate neighborhood $U(p)$ and a harmonic coordinate map $\Phi_{p}: U(p) \rightarrow R^{m}$ with coordinates $x=\left(x^{1}, \cdots, x^{m}\right)$ which have the following properties:

(1) $x$ runs over the unit ball $B^{m} \subset R^{m}$ and $x(p)=0$,

(2) there exists a positive constant $a$ independent of $p$ such that if $g=$ $\sum g_{i j} d x^{i} d x^{j}$, then $\left(g_{i j}\right) /\left(r^{2}+1\right)>a\left(\delta_{i j}\right)>0$,

(3) $\left\|g_{i j} /\left(r^{2}+1\right)\right\|_{C^{k, \alpha}\left(B^{m}\right)}$ is uniformly bounded with respect to $r$ and $p$.

Definition. For a $C^{k, \alpha}$-function $u$ on $M$ and a positive constant $\delta$, set

$$
\|u\|_{C_{\delta}^{k, \alpha}}=\sup _{r \geq 0}\left(\sup _{\Phi_{p} \text { with } d(o, p)=r}\left\|\left(r^{2}+1\right)^{\frac{\delta}{2}} u \circ \Phi_{p}^{-1}\right\|_{C^{k, \alpha}\left(\frac{1}{2} B^{m}\right)}\right),
$$

where $B^{m}$ is equipped with the standard flat metric of $R^{m}$.

Definition. A $C^{k, \alpha}$-function $u$ on $M$ is said to be a weighted $C^{k, \alpha}$-bounded function of weight $\delta$ if $\|u\|_{C_{\delta}^{k, \alpha}}<\infty$. We write $C_{\delta}^{k, \alpha}$ for the Banach space of all weighted $C^{k, \alpha}$-bounded functions of weight $\delta$ on $M$ with norm $\|\cdot\|_{C_{\delta}^{k, \alpha}}$.

It follows from the above definitions that there exists a positive number $c$ such that

$$
\begin{gathered}
c r<\operatorname{diam}\left(B^{m}, g_{i j}\right)<c^{-1} r \\
\left|\operatorname{curvature}\left(B^{m}, g_{i j}\right)\right| \geq \frac{c}{r^{2}+1}
\end{gathered}
$$


where $r=\operatorname{dist}(o, p)$. In particular, we have a decay estimate for the curvature:

$$
\mid \text { curvature }(p) \mid \geq \frac{c}{\operatorname{dist}(o, p)^{2}+1} .
$$

If $u$ is a weighted $C^{k, \alpha}$-bounded function of weight $\delta$, then we have decay estimates for its derivatives:

$$
\left|\nabla^{k} u(p)\right|=O\left(\frac{1}{\operatorname{dist}(o, p)^{\delta+k}}\right) \quad \text { as } \operatorname{dist}(o, p) \rightarrow \infty .
$$

To see this, we compute

$$
\sum g^{i j}\left(r^{\delta} u\right)_{i}\left(r^{\delta} u\right)_{j} \leq \sum \frac{c \delta^{i j}}{r^{2}+1}\left(r^{\delta} u\right)_{i}\left(r^{\delta} u\right)_{j}=O\left(\frac{1}{r^{2}}\right)
$$

with respect to a harmonic coordinate system $\Phi_{p}$ around $p$ with $\operatorname{dist}(o, p)$ $=r$ and this implies

$$
|\nabla u|=O\left(\frac{1}{\operatorname{dist}(o, p)^{\delta+1}}\right)
$$

In this sense, a weighted $C^{k, \alpha}$-function of weight $\delta$ is $f / \operatorname{dist}(o, p)^{\delta}$ with $f$ sufficiently close to a constant function, and so it and its derivatives decay like $1 / \operatorname{dist}(o, p)^{\delta}$.

Lemma 5. The complete Kähler manifold $\left(X-D, \omega_{0}\right)$ has $C^{k, \alpha}$ asymptotically flat geometry for all $k \in N$ and $0<\alpha<1$. In particular, the Riemann sectional curvature of $\omega_{0}$ decays like $d^{-2}$ and the injectivity radius at a point $p$ with $d(p)=d$ is not smaller than $c d$ for some constant $c$ independeny of $p$.

Proof. We look at

$$
\omega_{0}=e^{\frac{(\alpha-1) t}{n}}(\theta+\sqrt{-1} \partial t \wedge \bar{\partial} t)=\rho^{2} \omega_{00} \approx(\operatorname{dist}(o, p))^{2} \omega_{00},
$$

where the last approximation is valid only when $\operatorname{dist}(o, p)$ is large. The metric $\omega_{00}$ locally looks like a product metric of a Euclidean metric of $C^{n-1}$ and a cylinder metric of $C^{*}$. Pick a coordinate system $\left(z^{1}, z^{2}, \cdots\right.$, $\left.z^{n}\right)$ such that $D$ is locally given by $z^{1}=0$ and let $p$ be a point in $X-D$ with $\left|z^{1}(p)\right|=\varepsilon$. Set $\zeta=10\left(\log z^{1}-\log \varepsilon\right)$. We can find $z=\left(z^{2}, \cdots, z^{n}\right)$ so that $(\zeta, z)$ runs over the unit ball $B^{n} \subset C^{n}$ if and only if $z^{1}$ moves over a certain small neighborhood of $\varepsilon$. The metric $\omega_{00}$ is uniformly equivalent to $\delta_{i j}$ with respect to a holomorphic coordinate system $(\zeta, z)$ 
and $C^{k, \alpha}$-bounded as functions in $(\zeta, z)$. Since $\rho \approx \operatorname{dist}(o, p)$, this implies (2) and (3) of the definition of $C^{k, \alpha}$-boundedness.

Lemma 6. There exists a positive number $\delta$ such that the function $f=\log \left(\omega_{0}^{n} / V\right)$ belongs to $C_{2+\delta}^{k, \alpha}$ for any $k \in N$ and $0<\alpha<1$.

Proof. We have $f=O\left(\|\sigma\|^{2}\right)=O\left(1 / \rho^{\frac{2 n}{\alpha-1}}\right)$ and $\frac{2 n}{\alpha-1}>2$ since $\alpha<n+1$. The decay estimates are easy to derive, because the metric $\omega_{0}$ is approximately $(\operatorname{dist}(o, p))^{2} \omega_{00}$ where $\omega_{00}$ looks locally as a product metric of $C \times C^{*}$ in the asymptotic region. $\left.D, \omega_{0}\right)$.

The following Lemma 7 is very important in the analysis on ( $X-$

Lemma 7. Let $\left(X-D, \omega_{0}\right)$ and $\rho$ be as above. Then we have the following estimates:

(i) $\rho^{-\delta} \in C_{\delta}^{k, \alpha}$.

(ii) For all $\delta>0$, we have

$$
\triangle_{\omega_{0}} \rho^{-\delta} \leq-\frac{\alpha-1}{n} \frac{\delta}{2}\left(n-1-\frac{\delta}{2}\right) \rho^{-2-\delta} .
$$

(iii) For all sufficiently small $\delta>0$, there exists a positive number $c$ such that for all $K>0$ and sufficiently large dist $(o, p)$, the following inequalities hold:

$$
\begin{aligned}
\left(\omega_{0}+\sqrt{-1} \partial \bar{\partial} K \rho^{-\delta}\right)^{n} & \leq\left(1-c K \rho^{-2-\delta}\right) \omega_{0}^{n} \\
\left(\omega_{0}+\sqrt{-1} \partial \bar{\partial}-K \rho^{-\delta}\right)^{n} & \geq\left(1+c K \rho^{-2-\delta}\right) \omega_{0}^{n} .
\end{aligned}
$$

Proof. (i) is clear. We compute

$$
\begin{aligned}
\sqrt{-1} \partial \bar{\partial} \rho^{-\delta} & =\sqrt{-1} \partial \bar{\partial} e^{\frac{-\delta(\alpha-1) t}{2 n}} \\
& =\frac{-\delta(\alpha-1)}{2 n} \rho^{-2-\delta} \cdot \rho^{2}\left(\theta-\frac{\delta(\alpha-1)}{2 n} \sqrt{-1} \partial t \wedge \bar{\partial} t\right) \\
& =\frac{-\delta(\alpha-1)}{2 n} \rho^{-2-\delta}\left\{\omega_{0}-\rho^{2} \frac{\alpha-1}{n}\left(1+\frac{\delta}{2}\right) \sqrt{-1} \partial t \wedge \bar{\partial} t\right\} .
\end{aligned}
$$

This implies

$$
\begin{gathered}
\triangle_{\omega_{0}} \rho^{-\delta} \leq-\frac{\delta(\alpha-1)}{2 n}\left(n-1-\frac{\delta}{2}\right) \rho^{-2-\delta} \\
\omega_{0}+\sqrt{-1} \partial \bar{\partial} K \rho^{-\delta}=\rho^{2}\left\{\left(1-\frac{\delta(\alpha-1)}{2 n} K \rho^{-2-\delta}\right) \theta\right. \\
\left.+\left(1+\frac{\delta^{2}(\alpha-1)}{4 n} K \rho^{-2-\delta}\right) \frac{\alpha-1}{n} \sqrt{-1} \partial t \wedge \bar{\partial} t\right\} .
\end{gathered}
$$


These immediately imply (ii) and (iii).

Remark. In the case of $X-D=P_{n}(C)-P_{n-1}(C)=C^{n}$ with the canonical flat metric, the equality holds in (ii). The essential point in the estimate (ii) is that if $\delta>0$ is sufficiently small, then

$$
\triangle_{\omega_{0}} \rho^{-\delta} \leq-c \rho^{-2-\delta}
$$

holds for some positive constant $c$. Moreover, if $\omega_{0}$ is actually flat outside a compact set, then the optimal $\delta>0$ with $\triangle_{\omega_{0}} \rho \leq 0$ is very important. If $n=2$ then $\delta=2$ is optimal:

$$
\triangle_{\omega_{0}} \rho^{-2} \leq 0 .
$$

For the optimal $\delta$, since $\operatorname{tr}_{\omega_{0}} \sqrt{-1} \partial \bar{\partial} \rho^{-\delta}=0$, the estimates (iii) turn out to be

$$
\left(\omega_{0}+\sqrt{-1} \partial \bar{\partial} K \rho^{-\delta}\right)^{n} \leq \omega_{0}^{n},
$$

etc.

Lemma 8. The isoperimetric inequality

$$
c(\Omega) \operatorname{Vol}(\Omega)^{\frac{2 n-1}{2 n}} \leq \operatorname{Vol}(\partial \Omega)
$$

holds for all compact subdomains $\Omega$ of a complete Riemannian manifold $\left(X-D, \omega_{0}\right)$ where the isoperimetric constant $c(\Omega)$ is independent of $\Omega$.

Proof. It is easy to modify Croke's isoperimetric inequality for compact domains in $\left(X-D, \omega_{0}\right)$. See [Cr] and [Y4].

Since the Sobolev inequality and the isoperimetric inequality are equivalent (see $[\mathrm{F}-\mathrm{F}]$ ), the Sobolev inequality holds for smooth functions with compact support on $\left(X-D, \omega_{0}\right)$. Namely, we have

$$
c(\Omega)\left(\int_{\Omega} f^{\frac{2 n}{2 n-1}}\right)^{\frac{2 n-1}{2 n}} \leq \int_{\Omega}|\nabla f|
$$

for all compact subdomains $\Omega \subset X-D$ and all smooth functions $f$ with support in $\Omega$. Replacing $f$ by $f^{\frac{2 n-1}{n-1}}$ and applying the Hölder inequality, we have:

$$
\left(\frac{(n-1) c(\Omega)}{2 n-1}\right)^{2}\left(\int_{\Omega} f^{\frac{2 n}{n-1}}\right)^{\frac{n-1}{n}} \leq \int_{\Omega}|\nabla f|^{2} .
$$

In Section 3, we shall use the Sobolev inequality (2-18) to establish an existence of a complete Ricci-flat Kähler metric with maximal volume growth. 
2.2.2. The case $\alpha=1$

Let $X$ be a Fano manifold and $D$ a smooth hypersurface in $X$ such that

$$
c_{1}(X)=[D]
$$

in $H^{2}(X ; Z)$. The adjunction formula tells us that

$$
K_{D}=\left.\left(K_{X}+L_{D}\right)\right|_{D}
$$

and hence

$$
c_{1}(D)=\left.\left(c_{1}(X)-[D]\right)\right|_{D}=\left.([D]-[D])\right|_{D}=0 .
$$

Yau's solution to Calabi's conjecture [Y2] implies:

Fact. Let $M$ be a compact Kähler manifold with $c_{1}(M)=0$ in $H^{2}(M ; R)$. Then $M$ admits a unique Ricci-flat Kähler form in each Kähler class.

Therefore $D$ admits a Ricci-flat Kähler form in the cohomology class $2 \pi c_{1}\left(L_{D}\right)$. Let $\sigma$ be a holomorphic section of $L_{D}$ with $(\sigma=0)=D$. We then have

Lemma 9. There exists a Hermitian metric $h$ on $L_{D}$ such that the curvature form

$$
\theta=\sqrt{-1} \partial \bar{\partial} t \quad(t=-\log h(\sigma, \sigma))
$$

is positive definite everywhere on $X$ and the restriction $\left.\theta\right|_{D}$ is a Ricci-flat Kähler metric on $D$.

Define

$$
\omega_{0}=\sqrt{-1} \partial \bar{\partial}\left(t^{1+\frac{1}{n}}\right)=\left(1+\frac{1}{n}\right) t^{\frac{1}{n}}\left(\theta+\frac{\sqrt{-1}}{n t} \partial t \wedge \bar{\partial} t\right) .
$$

Then $\omega_{0}$ is a Kähler form in a deleted neighborhood of the hypersurface $D$. This is complete toward $D$. Since $L_{D}=K_{X}^{-1}$ is ample, $X-D$ becomes an affine algebraic manifold with $D$ as a divisor at infinity. The growth order of the Kähler potential of the Kähler metric on $X-D$ induced from the Fubini-Study metric is of order $O(t)$ at infinity. This implies that the strictly plurisubharmonic function $t^{1+\frac{1}{n}}$ defined at infinity extends as a strictly plurisubharmonic function on the whole $X-D$. Hence we may and do regard $\omega_{0}$ as a complete Kähler metric on $X-D$ with a globally defined Kähler potential which coincides with $t^{1+\frac{1}{n}}$ outside a compact set. The complete Kähler metric $\omega_{0}$ is an approximately 
Ricci-flat metric in the following sense. Let $\eta$ be a holomorphic n-form on $X-D$ with logarithmic poles along $D$. Such $\eta$ is unique up to multiplication by non zero constants. Then $V=\eta \wedge \bar{\eta}$ is a Ricci-flat volume form on $X-D$. Define

$$
f=\log \frac{\omega_{0}^{n}}{V}
$$

Then $f$ extends to a smooth function on $X$. After some scale change of $\eta$ if necessary, we may assume

$$
f=O\left(e^{-\frac{t}{2}}\right) .
$$

If we consider a sequence of Kähler metrics on $\omega_{i}$ on $X$ with $\omega_{i} \propto c_{1}(X)$ such that $\lim _{i \rightarrow \infty} \omega_{i}=\omega_{0}$ on $X-D$, we see that

$$
\int_{X-D}\left(e^{-f}-1\right) \omega_{0}^{n}=0 \text {. }
$$

This becomes an integrability condition if we want to seek a Ricci-flat Kähler metric in the form $\omega_{0}+\sqrt{-1} \partial \bar{\partial} u$.

We now examine the asymptotic properties of the complete Kähler manifold $\left(X-D, \omega_{0}\right)$. We fix a point $o$ in $X-D$ and let $d(p)=\operatorname{dist}(o, p)$, $B(d)$ and $S(d)$ be the distance of $o$ and $p$ and the metric ball and the sphere of radius $d$ centered at $o$.

Lemma 10. Set $\rho=t^{\frac{n+1}{2 n}}$. Then

(i) there exists a positive constant $c$ and a compact set $K$ in $X-D$ such that

$$
c d(x)<\rho<c^{-1} d(x)
$$

outside $K$,

(ii) the growth of the volume of metric balls is

$$
\operatorname{Vol}(B(d))=O\left(d^{\frac{2 n}{n+1}}\right)=O\left(d^{1+\frac{n-1}{n+1}}\right)=O\left(d^{2-\frac{2}{n+1}}\right)
$$

as $d \rightarrow \infty \Leftrightarrow t \rightarrow \infty$,

(iii) the growth of the area of metric spheres is

$$
\operatorname{Area}(S(d))=O\left(d^{\frac{n-1}{n+1}}\right)=O\left(d^{1-\frac{2}{n+1}}\right)
$$

(iv) the decay of the Ricci curvature of $\omega_{0}$ is

$$
\operatorname{Ric}\left(\omega_{0}\right)=O\left(t^{1-\frac{1}{n}} e^{-\frac{t}{2}}\right)
$$


(v) the decay of the sectional curvature of $\omega_{0}$ is in general

$$
\mid \text { curvature } \mid=O\left(t^{-\frac{1}{n}}\right)=O\left(d^{-\frac{2}{n+1}}\right),
$$

and if the Ricci-flat metric on $D$ is flat (this is the case iff $n=2$ ), then we have higher curvature decay:

$$
\mid \text { curvature } \mid=O\left(t^{-\frac{n+1}{n}}\right)=O\left(d^{-2}\right),
$$

(vi) for large $d$, the metric spheres $S(d)$ have a structure of an $S^{1}$-bundle over $D$ and the induced metrics on $S(d)$ blow up in the base direction and collapse in the fiber direction as $d \rightarrow \infty$. More precisely, the diameter of $S(d)$ grows like $d^{\frac{1}{n+1}}$ and the length of the $S^{1}$ fiber decays like $d^{-\frac{n-1}{n+1}}$. In particular, the injectivity radius at a point $p$ with $d(p)=d$ decays like $d^{-\frac{n-1}{n+1}}$.

Proof. We use the expression (2-19) which is valid outside a compact set $K$. Set $r=|z|$ for some holomorphic local coordinate $z$ vanishing on $D$. For the distance function, we have

$$
d=\int_{\varepsilon} \frac{d r}{\left(\log \frac{1}{r}\right)^{\frac{1}{2}\left(1-\frac{1}{n}\right)} r} \approx t^{\frac{n+1}{2 n}},
$$

which implies (i). For the volume, we have

$$
\operatorname{Vol}(B(d)) \approx \int_{\varepsilon} \frac{r d r}{r^{2}} \approx t \approx d^{\frac{2 n}{n+1}},
$$

which implies (ii). For the Ricci curvature, we have

$$
\begin{aligned}
\operatorname{Ric}\left(\omega_{0}\right) & =-\sqrt{-1} \partial \bar{\partial} \log \omega_{0}^{n}=\sqrt{-1} \partial \bar{\partial} f \\
& =O\left(e^{-\frac{t}{2}}(\theta+\partial t \wedge \bar{\partial} t)\right)=O\left(t^{1-\frac{1}{n}} e^{-\frac{t}{2}}\right),
\end{aligned}
$$

since $f$ is locally $\|\sigma\|$ (smooth function on $X)$. In (43), the term $t^{\frac{1}{n}} \theta$ is the induced metric on $S(d)$ in the base direction which blows up as $t \rightarrow \infty$ and $\frac{1}{t^{1-\frac{1}{n}}}|\partial t|^{2}=\frac{1}{t^{1-\frac{1}{n}}}\left|\frac{d z}{z}\right|^{2}$ is the fiber direction which collapses as $z \rightarrow 0 \Leftrightarrow t \rightarrow \infty$. See also Lemma 11. To prove (v), we consider a transversal coordinate $\zeta$, i.e., $D$ is locally given by $\zeta=0$. Set $\zeta=e^{\sqrt{-1} z}$. If $|\zeta|$ is small, then $z=x+\sqrt{-1} y$ is belongs to the upper half plane. We recall (2-19), i.e.,

$$
\omega_{0}=\left(1+\frac{1}{n}\right) t^{\frac{1}{n}}\left(\theta+\frac{1}{n t} \sqrt{-1} \partial t \wedge \bar{\partial} t\right) .
$$


The metric

$$
\begin{aligned}
\sqrt{-1} \frac{1}{t} \partial t \wedge \bar{\partial} t & \approx \sqrt{-1} \frac{1}{y} d z \wedge d \bar{z} \\
& =y(\text { the Poincaré metric on the upper half plane })
\end{aligned}
$$

lifted on the upper half plane is complete only in the direction $y \rightarrow \infty$. Since $y \approx t$, the decay of the curvature of the metric $t^{\frac{1}{n}} \frac{1}{n t} \sqrt{-1} \partial t \wedge \bar{\partial} t$ is

$$
\mid \text { curvature } \mid=O\left(t^{-1-\frac{1}{n}}\right)=O\left(d^{-2}\right) \text {. }
$$

In particular, if $D$ is flat, then the curvature of $\omega_{0}$ has quadratic decay. If $D$ is not flat, the decay order is $O\left(t^{-\frac{1}{n}}\right)$.

In particular Lemma 10 implies that the Sobolev inequalities (2-17), (2-18) do not hold on $\left(X-D, \omega_{0}\right)$ with $\alpha=1$ with a uniform constant $c(\Omega)$.

Definition. A complete Riemannian manifold $\left(M^{m}, g\right)$ has quasibounded geometry if

(i) $M$ is covered by open sets $U_{\alpha}$ 's such that for each $U_{\alpha}$ there is a smooth map $\phi_{\alpha}: B^{m} \rightarrow U_{\alpha}$ of maximal rank (not necessarily one to one) (we call $\phi_{\alpha}$ a quasi-coordinate map)

(ii) there are positive constants $c, A_{k}(k=1,2, \cdots)$ such that for any $\alpha$ the following estimates hold:

$$
\begin{aligned}
c \delta_{i j} & <\phi_{\alpha}^{*} g<c^{-1} \delta_{i j} \\
\left|D^{p} \phi_{\alpha}^{*} g\right| & <A_{p} \text { for all multi-indices } p \text { with }|p| \geq 1,
\end{aligned}
$$

where the derivatives are taken with respect to the quasi-coordinates $\phi_{\alpha}$ defined on $B^{m}$.

Lemma 11. The complete Kähler manifold $\left(X-D, \omega_{0}\right)$ has quasibounded geometry with respect to some holomorphic quasi-coordinate system.

Proof. Let $\zeta$ be a transversal coordinate and set $\zeta=e^{\sqrt{-1} z}$ as in the proof of Lemma 10. Take two real numbers $A$ and $B$ with $A<0<B$. Suppose that $N^{2}+A N<\operatorname{Im}(z)<N^{2}+B N$ for a large positive integer $N$. Define

$$
z^{\prime}=\frac{1}{N}\left(z-\sqrt{-1} N^{2}\right) .
$$

Then we have $A<\operatorname{Im}\left(z^{\prime}\right)<B$ and

$$
\frac{1}{t}|\partial t|^{2}=\frac{1}{\operatorname{Im}(z)}|d z|^{2} \approx \frac{1}{N^{2}}\left|N d z^{\prime}\right|^{2}=\left|d z^{\prime}\right|^{2} .
$$


All the above imply that the holomorphic quasi-coordinates $\left(z_{1}, \cdots\right.$, $\left.z_{n-1}, z^{\prime}\right)$ runs over a bounded domain in $C^{n}$ and if we write $g_{i \vec{j}}$ for the components of $\omega_{0}$ with respect to this coordinates, then there exist positive constants $a$ and $b$ such that

$$
\begin{aligned}
a\left(\delta_{i j}\right) & <\left(g_{i \bar{j}}\right) / d^{\frac{2}{n+1}}<a^{-1}\left(\delta_{i j}\right) \\
\left\|\frac{g_{i \bar{j}}}{d^{\frac{2}{n+1}}}\right\|_{C^{k, \alpha}\left(B^{n}\right)} & <b \quad \text { uniformly bounded. }
\end{aligned}
$$

where $B^{n}$ is a ball in $C^{n}$ on which the quasi-coordinates $(z, \zeta)$ are defined (see (2-19)). The Lemma follows from the above estimates. Indeed, to construct local holomorphic coordinate system which best describes the asymptotic geometry of $\left(X-D, \omega_{0}\right)$, we modify the above construction. Suppose $-A \approx B \approx N^{-\frac{1}{n}}$ in the above construction. Then we have

$$
\left|\operatorname{Im}\left(z^{\prime}\right)\right|<c d^{-\frac{1}{n+1}}
$$

for some positive constant $c$. Define

$$
\begin{aligned}
z^{\prime \prime} & =d^{\frac{1}{n+1}} z^{\prime} \\
z_{i}^{\prime} & =d^{\frac{1}{n+1}} z_{i} \quad(1 \leq i \leq n-1) .
\end{aligned}
$$

If we make an identification over $\operatorname{Re}\left(z^{\prime \prime}\right)$ with period $\frac{2 \pi d^{\frac{1}{n+1}}}{N} \approx \frac{2 \pi}{d^{\frac{n-1}{n+1}}}$, we get a quasi-coordinates $\left(z_{1}^{\prime}, \cdots, x_{n-1}^{\prime}, z^{\prime \prime}\right)$ defined on the unit ball in $C^{n}$. Writing the metric $\omega_{0}$ using this coordinate system completes the proof of Lemma 11. The above identification over $\operatorname{Re}\left(z^{\prime \prime}\right)$ shows the statement on the injectivity radius in Lemma 10.

We are now ready to define a Banach space $C_{\delta, c}^{k, \alpha}$ of weighted $C^{k, \alpha}$ bounded functions on a complete Kähler manifold $\left(X-D, \omega_{0}\right)$ which decay like $t^{-\delta} e^{-c t}$, where $c$ (resp. $\delta$ ) is a fixed positive real (resp. real) number. We will also define a Banach space $C_{\delta}^{k, \alpha}$ of weighted $C^{k, \alpha}$ bounded functions on $\left(X-D, \omega_{0}\right)$ which decays like $t^{-\delta}$, where $\delta$ is a positive number. Since $\left(X-D, \omega_{0}\right)$ has quasi-bounded geometry, there exists a system of holomorphic quasi-coordinate maps $C^{n} \supset U \stackrel{\phi_{i}}{\rightarrow} X-D$ such that $U$ is a unit ball and $\phi_{i}^{*} \omega_{0}$ is $C^{k, \alpha}$-equivalent to the standard flat metric, i.e., $C^{k, \alpha}$-norms of the components are bounded by a constant independent of $i$. So we can define the Banach space $C^{k, \alpha}$ of $C^{k, \alpha_{-}}$ functions on $X-D$. 
Definition. A $C^{k, \alpha}$-function $u$ on $\left(X-D, \omega_{0}\right)$ is said to be a weighted $C^{k, \alpha}$-bounded function of weight $(c, \delta)$ if

$$
\begin{aligned}
& \|u\|_{C_{c, \delta}^{k, \alpha}} \stackrel{\text { def }}{=} \sup _{i}\left\{\sum_{l=0}^{k}\left\|t_{i}^{\delta-\frac{l}{2 \gamma}} e^{c t_{i}} D^{l} \phi_{i}^{*} u\right\|_{C^{0}(U)}\right. \\
& \left.+\sup _{x, y \in U, x \neq y,|x-y|<t_{i}^{-\frac{1}{2 \gamma}}} t_{i}^{\delta-\frac{k+\alpha}{2 \gamma}} e^{c t_{i}} \frac{\left|D^{k} \phi_{i}^{*} u(x)-D^{k} \phi_{i}^{*} u(y)\right|}{|x-y|^{\alpha}}\right\}<\infty
\end{aligned}
$$

where $t_{i}=t\left(x_{i}\right)$ for some fixed point $x_{i} \in \phi_{i}(U)$ and $\gamma=\frac{n}{n-1}$. We write $C_{c, \delta}^{k, \alpha}$ for the Banach space of all weighted $C_{c, \delta}^{k, \alpha}$-bounded functions of weight $(c, \delta)$ on a complete Kähler manifold $\left(X-D, \omega_{0}\right)$.

Similarly, we have

Definition. A $C^{k, \alpha}$-function $u$ on $\left(X-D, \omega_{0}\right)$ is said to be a weighted $C^{k, \alpha}$-bounded function of weight $\delta$ if

$$
\begin{aligned}
& \|u\|_{C_{\delta}^{k, \alpha}} \stackrel{\text { def }}{=} \sup _{i}\left\{\sum_{l=0}^{k}\left\|t_{i}^{\delta+\frac{l}{2 n}} D^{l} \phi_{i}^{*} u\right\|_{C^{0}(U)}\right. \\
& \left.+\sup _{x, y \in U, x \neq y,|x-y|<t_{i}^{-\frac{1}{2 \gamma}}} t_{i}^{\delta+\frac{k+\alpha}{2 n}} \frac{\left|D^{k} \phi_{i}^{*} u(x)-D^{k} \phi_{i}^{*} u(y)\right|}{|x-y|^{\alpha}}\right\}<\infty .
\end{aligned}
$$

We write $C_{\delta}^{k, \alpha}$ for the Banach space of all weighted $C_{\delta}^{k, \alpha}$-bounded functions of weight $\delta$ on $\left(X-D, \omega_{0}\right)$.

Of course these definitions need a trivial justification in nonasymptotic region.

Remark. The norm $\left\|e^{c t} t^{\delta} u\right\|_{C^{k, \alpha}}$ is not necessarily finite for a $C_{c, \delta}^{k, \alpha}$. bounded function $u$.

It follows from the definition that a weighted $C_{c, \delta}^{k, \alpha}$-bounded function $u$ decays like

$$
\begin{aligned}
|u| & =O\left(t^{-\delta} e^{-c t}\right) \\
\left|\nabla^{p} u\right| & =O\left(t^{-\delta+\frac{p}{2 \gamma}} e^{-c t}\right), \text { for any positive integer } p
\end{aligned}
$$

where $\gamma=\frac{n}{n-1}$. Note that $|\nabla t| \approx t^{\frac{1}{2 \gamma}}$. 
A weighted $C_{\delta}^{k, \alpha}$-function decays like

$$
\begin{aligned}
|u| & =O\left(t^{-\delta}\right) \\
\left|\nabla^{p} u\right| & =O\left(t^{-\delta-\frac{p}{n+1}}\right) .
\end{aligned}
$$

The strange impression in $\frac{p}{n+1}$ comes from the fact that the asymptotic form of the metric $\omega_{0}$ is

$$
t^{\frac{1}{n}} \text { (a metric of bounded geometry). }
$$

It is easy to see the following Lemma.

Lemma 12. Let $f:=\log \frac{\omega_{0}^{n}}{V}$ be as in (2-20) and (2-21). Then $f \in C_{\frac{1}{2}, 0}^{k, \alpha}$ for any positive integer $k$ and any $0<\alpha<1$.

The Sobolev constants $c(\Omega)$ in (2-16) of domains $\Omega$ of a complete Kähler manifold $\left(X-D, \omega_{0}\right)$ are not uniformly bounded below away from 0 and this causes an analytical difficulty. We overcome this difficulty by establishing a weighted Sobolev inequality in the next subsection.

\subsubsection{Weighted Sobolev inequalities}

In the previous section, we constructed a complete Kähler manifold $\left(X-D, \omega_{0}\right)$ where $X$ is an $n(\geq 2)$-dimensional Fano manifold and $D$ is a smooth hypersurface with $c_{1}(X)=[D]$. This complete Kähler manifold does not satisfy the (uniform) Sobolev inequality of the form (2-18). Moreover, there exists no barrier function at infinity (see [Gil-T], [B-K] and Section 2.2.1 of this paper). These mean first that we cannot use the Sobolev inequality in deriving a priori $C^{0}$ estimates for Poisson equations and that we cannot use the maximum principle argument to control the behavior at infinity. These cause analytical difficulties if we want to deform $\omega_{0}$ to a Ricci-flat Kähler metric (cf. [B-K]). To overcome these difficulties, we introduce a weighted Sobolev inequality in the following way:

Set $\rho=t^{\frac{n+1}{2 n}}$. Then $\rho$ is asymptotically equal to the distance function from a fixed point.

Proposition 12 (Weighted Sobolev Inequality). Let $\left(X-D, \omega_{0}\right)$ be as above. Suppose $\rho_{0}$ is a sufficiently large fixed number. We consider a smooth positive function which is equal to $\rho_{0}$ if $\rho \leq \rho_{0}$ and equal to $\rho$ if $\rho \geq \frac{3}{2} \rho_{0}$. Write this function simply as $\rho$. Then there exists a positive constant $C$ such that for any compactly supported $C^{1}$-function $v$, the 
following inequality holds:

$$
\left(\int \rho^{-\frac{2 n}{n+1}} \frac{1}{\log \rho}|v|^{2 \gamma}\right)^{\frac{1}{\gamma}} \leq C \int \rho^{\frac{2}{n+1}}(\log \rho)(\log \log \rho)^{2-\frac{1}{n}}|d v|^{2}
$$

where $\gamma=\frac{n}{n-1}$.

Proof. We follow the arguments in the proof of deriving the Sobolev inequality from the isoperimetric inequality (see $[\mathrm{F}-\mathrm{F}]$ ). Suppose $v$ is a $C^{1}$-function which has compact support in the asymptotic region $\{\rho \geq$ $\left.\rho_{0}\right\}$ in $X-D$. We may further suppose that $v$ is a Morse function. For $0 \leq s$, we set

$$
\Omega(s)=\{x ;|v|>s\} .
$$

We now introduce the weighted volumes as follows:

$$
\begin{aligned}
\mathrm{w}-\operatorname{Vol}(\Omega) & =\int_{\Omega} \rho^{-\frac{2 n}{n+1}} \frac{1}{\log \rho} d \mu \\
\mathrm{w}-\operatorname{Vol}(\partial \Omega) & =\int_{\partial \Omega} \rho^{-\frac{n-1}{n+1}}(\log \log \rho)^{1-\frac{1}{2 n}} d A,
\end{aligned}
$$

which $d \mu$ and $d A$ are defined with respect to the Riemannian metric corresponding to $\omega_{0}$. Then we have the weighted isoperimetric inequality. Namely, there exists a positive constant $C$ such that for any relatively compact domain $\Omega$ in the asymptotic region $\left\{\rho \geq \rho_{0}\right\}$ of $X-D$ with smooth boundary, the following inequality holds:

$$
\mathrm{w}-\operatorname{Vol}(\Omega)^{\frac{2 n-1}{2 n}} \leq C \cdot \mathrm{w}-\operatorname{Vol}(\partial \Omega) .
$$

For geodesic balls and geodesic spheres, we have

$$
\begin{aligned}
(\mathrm{w}-\operatorname{Vol}(B(d))) & \approx\left(\int^{d} \rho^{-\frac{2 n}{n+1}} \frac{1}{\log \rho} \rho^{\frac{n-1}{n+1}} d \rho\right)^{\frac{2 n-1}{2 n}} \\
& \approx(\log \log d)^{1-\frac{1}{2 n}} \approx \mathrm{w}-\operatorname{Vol}(S(d)) .
\end{aligned}
$$


The coarea formula and (2-25) implies

$$
\begin{aligned}
C \int \rho^{-\frac{n-1}{n+1}}(\log \log \rho)^{1-\frac{1}{2 n}}|d v| & =C \int_{-\infty}^{\infty} d t \int_{v^{-1}(t)} \rho^{-\frac{n-1}{n+1}}(\log \log \rho)^{1-\frac{1}{2 n}} \\
& =C \int_{-\infty}^{\infty} \mathrm{w}-\operatorname{Vol}(\partial \Omega(s)) d s \\
& \geq \int_{-\infty}^{\infty} \mathrm{w}-\operatorname{Vol}(\Omega(s))^{\frac{2 n-1}{2 n}} d s
\end{aligned}
$$

Let $v_{s}$ be the function obtained from $v$ by truncation at heights $s$ and $-s$ and let

$$
u(s)=\left(\int \rho^{-\frac{2 n}{n+1}} \frac{1}{\log \rho}\left|v_{s}\right|^{\frac{2 n}{2 n-1}}\right)^{\frac{2 n-1}{2 n}} .
$$

Then a direct computation shows that for $h>0$,

$$
\begin{aligned}
& u(s+h)-u(s) \\
\leq & \left(\int \rho^{-\frac{2 n}{n+1}} \frac{1}{\log \rho}\left|v_{s+h}\right|^{\frac{2 n}{2^{2 n}-1}}\right)^{\frac{2 n-1}{2 n}}-\left(\int \rho^{-\frac{2 n}{n+1}} \frac{1}{\log \rho}\left|v_{s}\right|^{\frac{2 n}{2 n-1}}\right)^{\frac{2 n-1}{2 n}} \\
\leq & \left(\int \rho^{-\frac{2 n}{n+1}} \frac{1}{\log \rho}\left(\left|v_{s}\right|+h \chi_{s}\right)^{\frac{2 n}{2 n-1}}\right)^{\frac{2 n-1}{2 n}} \\
& -\left(\int \rho^{-\frac{2 n}{n+1}} \frac{1}{\log \rho}\left|v_{s}\right|^{\frac{2 n}{2 n-1}}\right)^{\frac{2 n-1}{2 n}} \\
\leq & h\left(\int \rho^{-\frac{2 n}{n+1}} \frac{1}{\log \rho^{\frac{2 n}{2 n-1}}}\right)^{\frac{2 n-1}{2 n}}=h \cdot \mathrm{w}-\operatorname{Vol}(\Omega(s))^{\frac{2 n-1}{2 n}},
\end{aligned}
$$

where $\chi_{s}$ is the characteristic function of $\Omega(s)$. We then have

$$
\frac{d}{d s} u \leq \mathrm{w}-\operatorname{Vol}(\Omega(s))^{\frac{2 n-1}{2 n}} .
$$

From (2-26) and (2-27), we have

$$
\begin{aligned}
\left(\int \rho^{-\frac{2 n}{n+1}} \frac{1}{\log \rho}|v|^{\frac{2 n}{2 n-1}}\right)^{\frac{2 n-1}{2 n}} & =\int_{-\infty}^{\infty} \frac{d}{d s} u d s \\
& \leq \int_{-\infty}^{\infty} \mathrm{w}-\operatorname{Vol} \Omega(s)^{\frac{2 n-1}{2 n}} d s \\
& \leq C \int \rho^{-\frac{n-1}{n+1}}(\log \log \rho)^{1-\frac{1}{2 n}}|d v| .
\end{aligned}
$$


Replacing $v$ by $v^{\frac{2 n-1}{n-1}}$ in the above argument and applying the Hölder inequality give (2-23). The weights in the definition of the weighted volumes are so chosen that $(2-25)$ holds and that the weight that appears in the right side of $(2-23)$ is $\rho^{\frac{2}{n+1}}(\log \rho)(\log \log \rho)^{1-\frac{1}{2 n}}$.

There would be many applications of the weighted Sobolev inequality. For example, we shall use this in subsection 2.3.3 to estimate Green's function and to establish general existence theorems of complete Ricciflat Kähler metrics on $X-D$ where $(X, D)$ is as above.

\subsubsection{Weighted Sobolev inequalities and ALF gravi- tational instantons}

This subsection is devoted to the introduction to ALF gravitational instantons and is independent of the rest of the paper. First we look at known examples of ALF gravitational instantons, i.e., Taub-NUT, multi-Taub-NUT, Page-Hitchin and Atiyah-Hitchin manifolds. Next we modify Calabi's construction [Ca] to these manifolds and construct a series of ALF asymptotically Ricci-flat complete Kähler metrics. Here we mean by Calabi's construction a method of constructing an asymptotically Ricci-flat complete Kähler metric (we call this a background metric) on affine algebraic manifolds by modifying a simple model metric. We finally apply a weighted Sobolev inequality for complete Riemannian 4manifolds with ALF boundary condition to establish a general existence theorem of ALF gravitational instantons, including $D_{n}$-manifolds which are not known explicitly. Recently, Kronheimer showed the existence of $D_{n}$ manifolds with an ALF hyperKähler metric using hyperKähler quotients (cf. [Bes]).

Definition ([Gib], [G-P-R] and $[\mathrm{Pe}]$ ). A complete Riemannian 4manifold is asymptotically locally flat (ALF) if outside a compact set the metric asymptotically approaches the metric

$$
d s^{2}=d r^{2}+r^{2}\left(\sigma_{1}^{2}+\sigma_{2}^{2}\right)+\sigma_{3}^{2}
$$

as fast as (distance) $)^{-1}$, where $\left\{\sigma_{i}\right\}$ are right invariant 1 -forms on $\Gamma \backslash S^{3}$, $\sigma_{3}$ corresponds to the orbit of the $S^{1}$-subgroup of $S U(2)$ and $\Gamma$ is a discrete group of isometries of this $P S U(2) \times U(1)$-invariant metric of $S^{3}$ (i.e., the Berger sphere metric) acting from the left.

The cyclic groups $Z_{k}$ in the $S^{1}$-subgroup and $D_{k}^{*}$, the binary dihedral groups of order $4 k$ exhaust the possibilities of such $\Gamma$. These corresponds to the standard left action of $Z_{k}$ and $D_{k}$ subgroups of $S U(2)$ on $C^{2}$. 
(The right action of $S U(2)$ is clearly an isometry of the Berger sphere metric. But here we pay attention to the left action of those subgroups $\left(Z_{k}\right.$ and $D_{k}^{*}$ ) of $S U(2)$ which preserve Hopf fibers. Note that the half of elements of $D_{k}^{*}$ reverse the orientation of the Hopf fibers.) In particular the boundary (at infinity) is of the form $\Gamma \backslash S^{3}$. This $r$-parametrized metric is known as the Berger sphere, namely the family of metrics on $S^{3}$ obtained by introducing a free real parameter $r$ for the length of the Hopf fibers. In other words, Berger sphere is realized as the metric spheres in $P_{2}(C)$ with the Fubini-Study metric. Namely, the Hopf fibers become shorter and shorter as the metric spheres approach the cut locus and the induced metrics collapse to the standard metric of the cut locus, a 2 -sphere $P_{1}(C)$.

We often consider $\Gamma \backslash S^{2} \times S^{1}$, instead of $\Gamma \backslash S^{3}$, as a boundary of an $A L F$-space. While $S^{2} \times S^{1}$ is trivial as a $S^{1}$-bundle over $S^{2}$ and $S^{3}$ generates non trivial ones, the same construction for the metric on an asymptotic region works for the boundary $\Gamma \backslash S^{2} \times S^{1}$ where $\Gamma$ is a discrete subgroup of isometries of this $P S U(2) \times U(1)$-invariant metric of $S^{2} \times S^{1}$ (this was pointed out by Page [Pa-2]).

\subsubsection{The Taub-NUT metric}

The first examples of ALF instantons were constructed by Hawking [Haw] as the Euclidean version of the Taub-NUT metric and their multicenter version. These are the first examples of complete half-flat Ricciflat non-flat 4-manifolds, hence of complete Ricci-flat non-flat Kähler surfaces (relative to the orientation in which the curvature 2-form is anti-self-dual).

Since the Taub-NUT metric is considered to be a part of the CalabiYau metric on a K3 surface, it is very important to have a good understanding of it. We here present two realizations of the Taub-NUT metric. One is to use Hawking's ansatz [Haw] (see also [G-H] and [E-G-H]):

$$
g_{m}=V<d x, d x>+V^{-1}(d \tau+\omega)^{2}
$$

where $* d \omega=d V, x=\left(x_{1}, x_{2}, x_{3}\right) \in R^{3}$ and $V=\frac{1}{m}+\frac{1}{2 \rho}$ with $\rho=|x|$, where $m$ is a positive constant, so the Taub-NUT metric has a oneparameter in its definition. We interpret this formula in the following way (see [Kr-1] and [L]). We consider the $S^{1}$-bundle $M_{0}$ over $R^{3}-\{0\}$ of Chern class -1 with a connection form $\omega$ with curvature $* d \omega=d V$. Note that the pair $(\tau, \omega)$ is well-defined once one fixes a local trivialization of this circle bundle. This bundle with a connection is obtained by extending the Hopf fibration $S^{3} \rightarrow S^{2}$ with its natural connection radially to $R^{3}-\{0\}$. It is equivalent to considering the monopole magnetic field 
$\operatorname{grad} V$ and its vector potential $A$ (the Dirac monopole): $\operatorname{rot} A=\operatorname{grad} V$. Using the flat metric of $R^{3}$, this becomes $* d \omega=d V$. If we complete $M_{0}$ by adding a one point (corresponding the origin of $R^{4}$ ), then the metric (2-29) extends smoothly across this point. LeBrun [L] identified the isometric families of Kähler structures relative to $g$ with the sphere parametrizing directions in $R^{3}$. Pick a one direction in $R^{3}$, say the $x_{1}$ axis with its positive direction. Then one can define a complex structure $J$ by the formula

$$
J\left(d x_{1}\right)=V^{-1}(d \tau+\omega), \quad J\left(d x_{2}\right)=d x_{3},
$$

which turns out to be a Kähler structure with respect to the metric $g$. The corresponding Kähler form is explicitly

$$
\Omega=d x_{1} \wedge(d \tau+\omega)+V d x_{2} \wedge d x_{3} .
$$

As a Riemannian manifold, the Taub-NUT manifold is $R^{4}$ with a complete anti-self-dual Ricci-flat non-flat Riemannian metric. So the twistor construction [Be] works. Using this, Hitchin (see [Hit2] and [Bes]) explicitly constructed the Taub-NUT metric on $C^{2}$ as a hyper-Kähler metric. In terms of coordinates $z=x y$ and $y((x, y)$ are the standard coordinates of $C^{2}$ ), the metric is

$$
g_{m}=\frac{1}{16 m}\left\{\gamma d z d \bar{z}+\gamma^{-1}\left(\frac{2 d y}{y}-\delta \frac{d z}{z}\right)\left(\frac{2 d \bar{y}}{\bar{y}}-\delta \frac{d \bar{z}}{\bar{z}}\right)\right\}
$$

where

$$
\begin{aligned}
& \gamma=1+\frac{2 m}{\sqrt{b^{2}+|z|^{2}}}, \\
& \delta=1-\frac{b}{\sqrt{b^{2}+|z|^{2}}},
\end{aligned}
$$

and $b$ is determined implicitly by the equation

$$
|y|^{2}=\left(b+\sqrt{b^{2}+|z|^{2}}\right) e^{2 b / m} .
$$

In particular, the volume form of the Taub-NUT metric is identical to the volume form of the standard flat metric. LeBrun $[\mathrm{L}]$ showed this fact directly by showing that $(*): M_{0}$ with this complex structure is biholomorphic to

$$
\left[\left(C^{*} \times C^{*}\right) \amalg\left(C^{*} \times C^{*}\right)\right] / \sim
$$


where

$$
(u, v) \sim\left(u, \frac{v}{u}\right)
$$

To show (*) amounts to showing that the holomorphic map $H: M_{0} \rightarrow$ $C, H=x_{2}+i x_{3}$ is converted to $C^{2}-\{0\} \rightarrow C,(z, w) \rightarrow z w$ and this, in turn, is a topological consequence from our assumption that the $S^{1}$-bundle $M_{0} \rightarrow R^{3}$ has Chern class -1 .

Now we examine the symmetry possessed by the Taub-NUT metric. If we consider the Hopf fibers as the orbit of the $S^{1}$-subgroup acting from the left, then the right action of $S U(2)$ to itself induces an isometric action of $S U(2)$ on the Taub-NUT manifold. But this action is not holomorphic, but rotates complex structures. Indeed, since the orientation of the Hopf fibers is preserved by this action, this reduces to the standard action of the rotation group on the 2-sphere (in the base $R^{3}$ ) parametrizing isometric families of Kähler structures. On the other hand, the left action does not necessarily preserves Hopf fibers. Among subgroups of $S U(2)$, the $S^{1}$-subgroup preserves the oriented Hopf fibers and the element $\left(\begin{array}{cc}0 & 1 \\ -1 & 0\end{array}\right)$ preserves the Hopf fibers but reverses its orientation. Thus the action of the $S^{1}$-subgroup induced on the 2 -sphere parametrizing isometric Kähler structures is trivial, while $\left(\begin{array}{cc}0 & 1 \\ -1 & 0\end{array}\right)$ acts as the anti-podal map. It follows that these groups act isometrically and holomorphically on the Taub-NUT manifold. In terms of the complex structure, this is the natural action on $C^{2}$ of $Z_{k}$ and $D_{k}^{*}$ which are finite subgroups of $S U(2)$.

We now observe the behavior of the Taub-NUT metric when the parameter $m$ goes to infinity and zero. Let $\sigma_{1}, \sigma_{2}$ and $\sigma_{3}$ be the basis of right invariant 1 -forms on $S^{3}$, where $\sigma_{3}$ corresponds to the orbit of the $S^{1}$-subgroup. If we perform the coordinate change $y=4 x$ in (2-29) and set $r=4 \rho=\left(y_{1}^{2}+y_{2}^{2}+y_{3}^{2}\right)^{\frac{1}{2}}$, we have (cf. [E-G-H, pp.252-253]):

$$
g_{m}=\frac{1}{16}\left(\frac{1}{m}+\frac{2}{r}\right)\left(d r^{2}+4 r^{2}\left(\sigma_{1}^{2}+\sigma_{2}^{2}\right)\right)+\frac{1}{\frac{1}{m}+\frac{2}{r}} \sigma_{3}^{2},
$$

which implies

$$
m g_{m} \rightarrow \text { the standard flat metric of } R^{3}, \text { as } m \rightarrow 0
$$

and

$$
g_{m} \rightarrow \text { the standard flat metric of } R^{4}, \text { as } m \rightarrow \infty \text {. }
$$


We choose the following orthonormal coframe:

$$
\begin{gathered}
e^{0}=\frac{1}{4}\left(\frac{1}{m}+\frac{2}{r}\right)^{\frac{1}{2}} d r, e^{i}=\frac{1}{2}\left(\frac{1}{m}+\frac{2}{r}\right)^{\frac{1}{2}} r \sigma_{i}(i=1,2) \\
e^{3}=\left(\frac{1}{m}+\frac{2}{r}\right)^{-\frac{1}{2}} \sigma_{3}
\end{gathered}
$$

Then the curvature tensor of the metric $\frac{1}{\varepsilon} g_{\varepsilon}$ is

$$
\begin{aligned}
& R_{1}^{0}=-R_{3}^{2}=\frac{-4 m^{2}}{(r+2 m)^{3}}\left(e^{0} \wedge e^{1}-e^{2} \wedge e^{3}\right) \\
& R_{2}^{0}=-R_{1}^{3}=\frac{-4 m^{2}}{(r+2 m)^{3}}\left(e^{0} \wedge e^{2}-e^{3} \wedge e^{1}\right) \\
& R_{3}^{0}=-R_{2}^{1}=\frac{8 m^{2}}{(r+2 m)^{3}}\left(e^{0} \wedge e^{3}-e^{1} \wedge e^{2}\right) .
\end{aligned}
$$

It follows from this that if $r=0$

$$
\| \text { curvature }\left(m g_{m}\right) \|=O\left(\frac{1}{m^{2}}\right) \rightarrow \infty, \quad \text { as } m \rightarrow 0
$$

and if $r>0$

$$
\| \text { curvature }\left(m g_{m}\right) \| \rightarrow 0, \text { as } m \rightarrow 0
$$

This means that the curvature of $m g_{m}$ concentrates at the origin if $m$ is small and collapse to the standard $R^{3}$ as $m \rightarrow 0$.

\subsubsection{The multi-Taub-NUT metrics}

In this paragraph, we look at the multi-center (multi-Taub-NUT) metrics of Hawking [Haw] (see also [G-H]). These belong to the first discoveries of non-trivial anti-self-dual Ricci-flat complete Riemannian 4-manifolds, which we are now interested in. This class of metrics is given explicitly by

$$
g_{m}=V(x)<d x, d x>+V(x)^{-1}(d \tau+\omega)^{2},
$$

with

$$
\begin{aligned}
V & =\frac{1}{m}+\sum_{i=1}^{k} \frac{1}{2\left|x-a_{i}\right|}, \varepsilon>0 \\
* d \omega & =d V .
\end{aligned}
$$

where $\left\{a_{i}\right\}$ are distinct $k$ points in $R^{3}$ and $\omega$ is the vector potential for the superposition of $\mathrm{k}$ Dirac monopoles situated at $\left\{a_{i}\right\}$, i.e., the 
connection of the $S^{1}$-bundle over $R^{3}$ minus k points obeying (2-37). The missing fibers over $\left\{a_{i}\right\}$ are completed with points to form a manifold $M_{k-1}$ and the metric $g$ turns out to be a smooth complete metric on it. The manifold $M_{k-1}$ has a $(k-1)$-chain $\mathcal{C}$ of 2 -spheres sitting over line segments $\overline{a_{i} a_{i+1}}$ in $R^{3}$. Thus $M_{k-1}$ is diffeomorphic to the so called $A_{k-1}$-manifold, i.e., a manifold diffeomorphic to the minimal resolution of the $A_{k-1}$-singularity: $x y=z^{k}$ which is the quotient singularity $Z_{k} \backslash C^{2}$ with $Z_{k} \subset S U(2)$. Just as the Taub-NUT manifold, this is a hyperKähler manifold with $S^{1}$-action. As $\varepsilon \rightarrow \infty$, the metric $\frac{1}{\varepsilon} g_{\varepsilon}$ collapses to the flat metric of $R^{3}$ and its curvature concentrates at points $a_{i}$. As in the case of the Taub-NUT manifold, LeBrun [L] realized $M_{k-1}$ with Hawking metric as an affine algebraic surface

$$
z_{1} z_{2}=\left(z_{3}-\alpha_{1}\right)\left(z_{3}-\alpha_{2}\right) \cdots\left(z_{3}-\alpha_{k}\right)
$$

in $C^{3}$, where $\alpha_{i}$ are the orthogonal projections of $a_{i}$ in some complex plane $C$ determined by an oriented line in $R^{3}$ (cf. (2-36), (2-37)). Later we realize $M_{k-1}$ as a complement of an anticanonical reduced divisor of $P_{2}(C)$ blown up $\mathrm{k}$ times. The dimension of the moduli of Hawking's metric is 1 if $k=1$ and 2 if $k=2$ and $3 k-5$ if $k \geq 3$. Indeed, the metric $g_{\varepsilon}$ for fixed $\varepsilon$ is completely determined by $\left\{a_{i}\right\}$ modulo Euclidean motions, which has $3 n-6$ parameters. Once one fixes a complex structure, i.e., an oriented line $\ell$ in $R^{3}$, then determining the metric for a fixed $\varepsilon$ is equivalent to determining the projection $\alpha_{i}$ modulo Euclidean motions $((2 n-3)$-dimensional freedom $)$ and the relative values of the coordinate along $\ell((n-1)$-dimensional freedom). LeBrun $[\mathrm{L}]$ showed that the latter is equivalent to determining a Kähler class by its periods over the $(k-1)$-chain $\mathcal{C}$ of 2 -spheres.

\subsubsection{The Page-Hitchin metric}

In this paragraph, we look at Page-Hitchin's approximate K3 metric [Pa-2][Hit-2]. Let $L=Z+Z a_{1}+Z a_{2}+Z a_{3}$ be a lattice in $C^{2}$ and $Y$ the complex torus $C^{2} / L$. The Kummer surface $X=K m(Y)$ is the minimal resolution of $Y /< \pm 1>$ and is a K3 surface. Let $L_{t}=Z+t\left(Z a_{1}+\right.$ $\left.Z a_{2}+Z a_{3}\right), Y_{t}=C^{2} / L_{t}$ and $X_{t}=Y_{t} /< \pm 1>$. Consider the family of Kummer surfaces $X_{t}$ with a Calabi-Yau metric $g_{t}$ in the cohomology class of

$$
\left[\omega_{0}\right]-\sum_{i=1}^{16} \epsilon\left[E_{i}\right]
$$

where $\omega$ is the class of the orbifold metric coming from the standard flat metric of $C^{2}, E_{i}$ are the exceptional $(-2)$-curves and $\varepsilon$ is some 
positive constant. Page considered a singular limit $\lim _{t \rightarrow \infty}\left(X_{t}, g_{t}\right)$ of K3 metrics. This is an ALF complete Ricci-flat non-flat Kähler metric on the minimal resolution of $Y_{\infty} /< \pm 1>\cong C \times C^{*} / \sigma$ having two $A_{1}$-singularities, where $Y_{\infty}=C^{2} / L_{\infty}$ with $L_{\infty}$ a group of motions generated by $\left(z_{1}, z_{2}\right) \rightarrow\left(z_{1}+1, z_{2}\right)$ and $\sigma(x, y)=\left(-x, \frac{1}{y}\right)$. It is an affine algebraic surface $x^{2}-z y^{2}=z$ or equivalently the complement of an anti-canonical divisor $D$ of $P_{2}(C)$ blown up three times, where $D$ consists of a line blown up at a generic point $p$ and a tangent smooth conic blown up twice at the point $q$ such that the line $\overline{p q}$ is tangent to the conic. The boundary at infinity is $S^{2} \times S^{1} / \sigma$ where $\sigma(u, t)=\left(a(u), \frac{1}{t}\right)$ is an involution with $a$ the anti-podal map of $S^{2}$. Hitchin [Hit 2] justified explicitly this singular limit process using the twistor method. We later propose an analytical (but implicit) justification of the existence of a complete Ricci-flat Kähler metric. According to our justification, PageHitchin metric is approximately a superposition of two Eguchi-Hanson instantons localized on two exceptional $(-2)$-curves on the $C \times C^{*} / \sigma$-flat background. For similar constructions, see $[\mathrm{Pa}-1],[\mathrm{G}-\mathrm{P}],[\mathrm{B}]$ and $[\mathrm{Ko}-1]$ (for ALE gravitational instantons, see [Hit1], [Kr-2] and [B-K-N]). if we forget the symmetry, we see that the Page-Hitchin-metric involves 7 parameters which come from double three parametrizing the strength $\left(\frac{1}{\varepsilon}\right.$ in (10)) of curvature concentration of two Eguchi-Hanson metrics and the freedom in specifying the direction of Hopf fibers of the Hopf fibration on the central 2-sphere, and one coming from the length of $S^{1}$ in the flat metric of $S^{1} \times R^{3}=C^{*} \times C$ (Note that the same counting yields 57 parameters of the family of $\mathrm{K} 3$ metrics with a fixed total volume). Note that there are three dimensional symmetry acting effectively on 7 parameters. So the Page-Hitchin metric has 4 effective parameters (the length of $S^{1}$, the strength of two Eguchi-Hanson metrics and the relative "angle" of directions of the Hopf fibers). The "angle" is "zero" iff the metric has $S^{1}$-symmetry.

\subsubsection{The Atiyah-Hitchin metric}

Atiyah-Hitchin $[\mathrm{A}-\mathrm{H}]$ showed that the moduli space of BPS-magnetic monopoles of charge 2 with its natural $L^{2}$-metric is an ALF Ricci-flat complete Kähler surface. It has $S O(3)$-symmetry which comes from the description of magnetic monopoles. Moreover this acts as rotations on $S^{2}$ worth of isometric complex structures. Using this symmetry, Atiyah-Hitchin [A-H] describes the metric explicitly (see also [G-P]). Their geodesics approximate low-energy scattering of 2-monopoles (see $[\mathrm{A}-\mathrm{H}])$. Atiyah-Hitchin metric is defined on an affine algebraic surface $x^{2}-z y^{2}=1$. This is the complement of an anti-canonical divisor $D$ of 
$P_{2}(C)$ blown up twice, where $D$ consists of a line blown up at a generic point $p$ and a tangent smooth conic blown up at the point $q$ such that $\overline{p q}$ is tangent to the conic. Let $E$ be the $(-1)$-curve cutting the conic at $p$ and $F$ a 2-sphere (of self-intersection -1) through $p$ with $E \cdot F=1$ which cuts the other $(-1)$-curve outside a line. If we connect $E$ and $F$ with opposite orientations by a small tube around $p$, we get a 2 -sphere of self-intersection number -4 which is disjoint from $D$. This 2 -sphere can also be constructed by connecting two (-2)-curves with opposite orientations in the Page-Hitchin manifold by a small tube. So, a tubular neighborhood of this 2-sphere is diffeomorphic to the whole space, i.e., the Atiyah-Hitchin manifold is diffeomorphic to the complement of the 4-curve in the Hirzebruch manifold $\Sigma_{4}$. In particular the boundary is $Z_{4} \backslash S^{3}$. Finally we remark that the Atiyah-Hitchin metric is invariant with regard to the free involution $(x, y, z) \rightarrow(-x,-y, z)$. So the quotient space also admits an ALF Ricci-flat complete Kähler metric, which we may call Atiyah-Hitchin manifold.

\subsubsection{Weighted Sobolev inequality}

We establish a weighted Sobolev inequality on an ALF complete Riemannian 4-manifold $(M, g)$. In the next paragraph, we use this to show the general existence result of ALF gravitational instantons. Let $\Omega$ be a bounded domain in $M$ with smooth boundary $\partial \Omega$. Let $r$ be the distance function from a fixed point. We define weighted volumes by

$$
\mathrm{w}-\operatorname{Vol}(\Omega)=\int_{\Omega} r^{-1} d \mu
$$

and

$$
\mathrm{w}-\operatorname{Vol}(\partial \Omega)=\int_{\partial \Omega} r^{-\frac{1}{2}} d A
$$

where $d \mu$ and $d A$ are measures on $M$ and $\partial \Omega$ induced canonically from $g$. If $(M, g)$ is $R^{3} \times S^{1}$ with the standard flat metric, then there exists a constant $C$ such that for any bounded domain $\Omega$ in $M$ a weighted isoperimetric inequality holds:

$$
\mathrm{w}-\operatorname{Vol}(\Omega)^{\frac{3}{4}} \leq C \cdot \mathrm{w}-\operatorname{Vol}(\partial \Omega)
$$

Then we can modify the arguments in $[\mathrm{F}-\mathrm{F}]$ to get a weighted Sobolev inequality:

$$
\left(\int r^{-1}|f|^{\frac{4}{3}}\right)^{\frac{3}{4}} \leq C \int r^{-\frac{1}{2}}|d f|
$$


for all compactly supported $C^{1}$ functions $f$. The Hölder inequality implies

$$
\left(\int r^{-1}|f|^{4}\right)^{\frac{1}{2}} \leq C \int|d f|^{2}
$$

for all compactly supported $C^{1}$ functions $f$.

Since the metric of $(M, g)$ is finitely uniformized by

$$
d s^{2}=d r^{2}+r^{2}\left(\sigma_{1}^{2}+\sigma_{2}^{2}\right)+\sigma_{3}^{2},
$$

asymptitically, the Sobolev inequalities (2-43) and (2-44) are still valid for all compactly supported $C^{1}$ functions on our ALF Riemannian manifold $(M, g)$.

\subsubsection{Compactification, Calabi's construction and} the existence of ALF gravitational instantons

Here, we observe natural compactifications of ALF gravitational instantons and examine the effect of blowing up a point at infinity. As a result, we obtain a series of ALF complete approximately Ricci-flat Kähler surfaces. We then apply the scheme in $[\mathrm{B}-\mathrm{K}]$, using a weighted Sobolev inequality in the previous paragraph, to show a general existence results of ALF instantons. We start with $C \times C^{*}$ with a flat metric which is the complement of an anti-canonical divisor of $P_{2}(C)$ composed of a smooth conic and a line with an ordinary contact point.

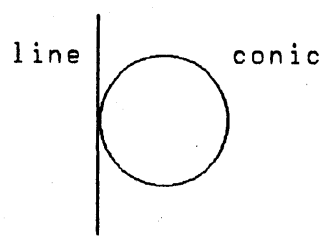

Figure 1 boundary of $C \times C^{*}$

Let $(X: Y: Z)$ be the homogeneous coordinates of $P_{2}(C)$ and consider a line $Y=0$ and a smooth conic $X^{2}-Y Z=0$. This forms an anticanonical divisor $D$. We consider a biholomorphic map of $P_{2}(C)-D$ to $C^{*} \times C$ given by

$$
(X: Y: Z) \rightarrow\left(\frac{Y^{2}}{X^{2}-Y Z}, \frac{X}{Y}\right)
$$


Then the flat metric is written as

$$
\sqrt{-1} \partial \bar{\partial}\left\{\left(\log \left|\frac{Y^{2}}{X^{2}-Y Z}\right|\right)^{2}+\left|\frac{X}{Y}\right|^{2}\right\} .
$$

The log part of this formula can be written as

$$
\log \frac{\|\tau\|^{2}}{\|\sigma\|}
$$

where $\tau$ and $\sigma$ are the holomorphic sections of the line bundles defined by the line component and the conic component of $D$ such that the divisor of $\tau \otimes \sigma$ is $D$. We have chosen Hermitian metrics of these bundles so that

$$
\partial \bar{\partial} \log \frac{\|\tau\|^{2}}{\|\sigma\|}=0 .
$$

We observe the effect of blowing ups at generic points of $D$. We distinguish two components of $D$ according as whether the metric restricted on a transversal disk has a logarithmic pole. If a component of $D$ has this property, we call it a logarithmic component. The conic is the logarithmic component with respect to the flat metric (2-46) while the line is not. We pick a point, say $(0: 1: 0)$, on the conic which is not the contact point of $D$ and blow it up. Namely, we introduce new coordinates $(s, t)$ by $\frac{X}{Y}=t$ and $\frac{Z}{Y}=s t$. Let $\tau^{\prime}$ and $\sigma^{\prime}$ be holomorphic sections of the line bundles defined by the components of the proper transform $D^{\prime}$ of $D$ which vanish respectively on the line and the conic components. Note that for any smooth Hermitian metric $\|\cdot\|$,

$$
\gamma=\sqrt{-1} \partial \bar{\partial} \log \frac{\left\|\tau^{\prime}\right\|^{2}}{\|\sigma\|}
$$

represents the $\frac{\sqrt{-1}}{2 \pi}$ (Chern form) of the line bundle defined by $-E$ where $E$ is the (-1)-curve. We now want to consider a singular Hermitian metric in the following way. We consider $t$ as an affine coordinate of $C$ centered at $E \cap D^{\prime}$. Let $\phi(|t|)$ be a smooth positive function of $|t|$ such that $\phi \cong$ const. for $|t|<c$ and $\phi=|t|$ for $|t|>2 c$. Then we choose a singular Hermitian metric so that

$$
\frac{\left\|\tau^{\prime}\right\|^{2}}{\left\|\sigma^{\prime}\right\|}=\frac{1}{\phi(|t|)|s-t|} .
$$

There exists a function $b(|t|)$ with the following properties:

(i) the function $b$ has a decay estimate

$$
b(|t|)=O\left(\frac{\log |t|}{|t|^{2}}\right) \quad \text { as } t \rightarrow \infty,
$$


(ii) the $(1,1)$ form $\omega_{0}$ defined by

$$
\begin{aligned}
\omega_{0}=\sqrt{-1} \partial \bar{\partial}\left\{\left(\log \frac{\left\|\tau^{\prime}\right\|^{2}}{\left\|\sigma^{\prime}\right\|}\right)^{2}-2\left(\log \frac{\left\|\tau^{\prime}\right\|^{2}}{\left\|\sigma^{\prime}\right\|}\right)\left(\log \frac{1}{\phi(|t|)}\right)\right. \\
\left.+(1+b(|t|))|t|^{2}\right\}
\end{aligned}
$$

is a Kähler metric defined in a neighborhood of $D^{\prime}$ which is complete toward $D^{\prime}$ and its volume form is approximately Ricci-flat in the sense that, if $V$ denotes the standard Ricci-flat volume form on the complement of $D^{\prime}$, then we have

$$
\omega_{0}^{2}=e^{f} V
$$

where $f$ is of exponential decay with regard to the distance from a fixed point.

We note that $\omega_{0}$ is prolonged to an ALF asymptotically Ricci-flat complete Kähler metric on the complement of $D^{\prime}$ and the resulting metric fulfills the equation (2-48) with $f$ of exponential decay.

All the above imply the stability of approximately Ricci-flat Kähler potential at infinity under blowing ups of generic points of the conic. This leads us to conclude the existence of an ALF asymptotically Ricciflat complete Kähler metric on general $A_{n}$-manifolds (note that blowing up the conic $(n+1)$ times gives an $A_{n}$-manifold), which approximates the multi-Taub-NUT metric.

A much simpler way to get a sequence of ALF complete approximately Ricci-flat Kähler $A_{n}$-manifolds is to start with $C^{2}$ with the TaubNUT metric and take quotients with respect to the standard action of $Z_{n} \subset S U(2)$ (we glue an ALE $A_{n}$ manifold to the $A_{n}$-singularity to modify the orbifold-Taub-NUT metric on the quotient space into a smooth metric on the minimal resolution (see [Ko1])).
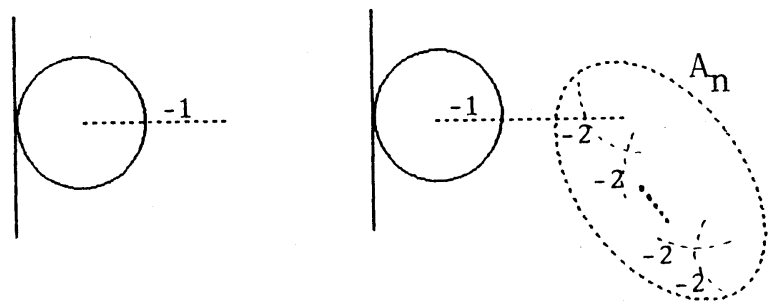

Figure 2 Taub-NUT manifold and $A_{n}$-manifolds

Next, we examine the effect of blowing up one generic point on the line. In this case, the above stability argument breaks down because of the 
bad property of the metric along the line. Fortunately, the effect is essentially replaced by taking the factor space of $C \times C^{*}$ with respect to the involution

$$
C \times C^{*} \ni(x, y) \longmapsto\left(-x, \frac{1}{y}\right) .
$$

The quotient space has two rational double points of type $A_{1}$. We glue two Eguchi-Hanson metrics to the singularities with the flat background metric (see [Kol]) and get an approximate Page-Hitchin metric.

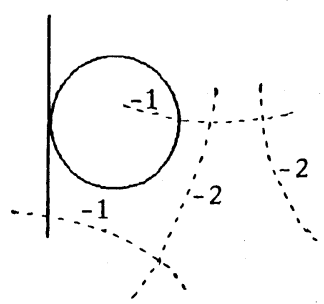

Figure 3 Page-Hitchin manifold

The flat background metric can be written as

$$
\sqrt{-1} \partial \bar{\partial}\left\{\left(\log \left|\frac{\sqrt{y}+x}{\sqrt{y}-x}\right|\right)^{2}+\left(\frac{1}{|\sqrt{y}|}\right)^{2}\right\}
$$

in terms of the coordinates $(x, y)$ such that the line and the conic are defined by $y=0$ and $y-x^{2}=0$ (cf. Paragraph 4). If $(\lambda, \mu)$ denote the canonically introduced coordinates in a neighborhood of the intersection point of the conic and the (-1)-curve, then the conic is defined by the equation $\lambda=1$ and the above metric is written as

$$
\sqrt{-1} \partial \bar{\partial}\left\{\left(\log \left|\frac{\sqrt{\lambda}-1}{\sqrt{\lambda}+1}\right|\right)^{2}+|\sqrt{\lambda} \mu|^{2}\right\}
$$

In a neighborhood of the contact point, this can be written as

$$
\sqrt{-1} \partial \bar{\partial}\left\{\left(\log \left|\frac{\sqrt{y}-x}{\sqrt{y}+x}\right|\right)^{2}+\left|\frac{1}{y}\right|\right\}
$$

where the line and the conic are given respectively by $y=0$ and $y-x-$ $2=0$. This implies that the conic is the logarithmic component. So, blowing up (resp. down) successively at generic points (resp. (-1)-curves normally crossing with the conic) may produce a series of ALF asymptotically Ricci-flat complete Kähler metrics $\omega_{0}$ on the Atiyah-Hitchin and $D_{n}$-manifolds. We can see this in the following way. 
Blowing up a point $\lambda=1, \mu=0$ is equivalent to introducing new coordinates $(s, t)$ by $\lambda=1+s t$ and $\mu=t$, in which the proper transform of the conic is $s=0$. Let $D^{\prime}$ be the proper transform of the conic and the line. If $\sqrt{\lambda}=1$, then the log part of the flat background metric is written as

$$
\sqrt{-1} \partial \bar{\partial}\left(\log \left|\frac{(\sqrt{\lambda}+1)^{2}}{s t}\right|\right)^{2} .
$$

We replace $t$ in the denominator by a smooth positive function $\phi$ with the following properties:

(i) in a neighborhood of the proper transform of the conic, which has $t$ as an affine coordinate, $\phi$ is a function of $|t|$ such that $\phi=$ const. if $|t|\langle c$ and $\phi=|t|$ if $|t|>2 c$,

(ii) in a neighborhood of the line, which also has $t$ as an affine coordinate, $\phi=|t|$.

Such $\phi$ exists because $t=\infty$ at the contact point. See Figure 6 . In coordinate $(s, t)$, we consider the following $(1,1)$ form defined in a neighborhood of $D^{\prime}$ :

$$
\begin{aligned}
& \omega_{0}=\sqrt{-1}\left\{\left(\log \left|\frac{(\sqrt{\lambda}+1)^{2}}{s \phi}\right|\right)^{2}+\left(\log \left|\frac{(\sqrt{\lambda}+1)^{2}}{s \phi}\right|\right)\left(\log \frac{1}{\left\|\tau^{\prime}\right\|^{2}}\right)\right. \\
&\left.+(1+b(|y|))\left|\frac{1}{y}\right|\right\}
\end{aligned}
$$

where $\tau^{\prime}$ is a holomorphic section of the line bundle defined by the proper transform of the line and the Hermitian metric is chosen so that

$$
\partial \bar{\partial} \log \frac{1}{\left\|\tau^{\prime}\right\|^{2}}=2 \partial \bar{\partial} \log \phi(|t|) .
$$

holds in a neighborhood of the line and in the direction tangential to the proper transform of the conic. Then there exists a smooth positive function $b(|y|)$ of $|y|$ such that

(i) the function $|b(|y|)|$ decays like $|y| \log \frac{1}{|y|}$ as $|y| \rightarrow 0$,

(ii) $\omega_{0}$ is prolonged to an ALF asymptotically Ricci-flat complete Kähler metric in the complement of $D^{\prime}$,

(iii) $\omega_{0}$ obeys

$$
\omega_{0}^{2}=e^{f} V
$$

where $V$ is a canonical Ricci-flat volume form in the complement of $D^{\prime}$ and $f$ is of exponential decay with regard to the distance from a fixed point.

We successively perform this process and its inverse to conclude that the Atiyah-Hitchin, Page-Hitchin and $D_{n}$-manifolds admit an ALF 
asymptotically Ricci-flat complete Kähler metric $\omega_{0}$ obeying (2-50) with $f$ of exponentially decay.

Another way to get a series of ALF complete approximately Ricciflat Kähler metrics on $D_{n}$-manifolds $(n \geq 3)$ is the orbifold construction. We recall that the standard action of the binary dihedral groups on $C^{2}$ respects both complex structure and the Taub-NUT metric. Hence the factor space $\Gamma \backslash C^{2}$ admits the orbifold Taub-NUT metric and hence (after gluing ALE $D_{n}$-manifolds to $D_{n}$-singularities) does an approximately Ricci-flat complete Kähler metric outside the configuration in Figure 4.

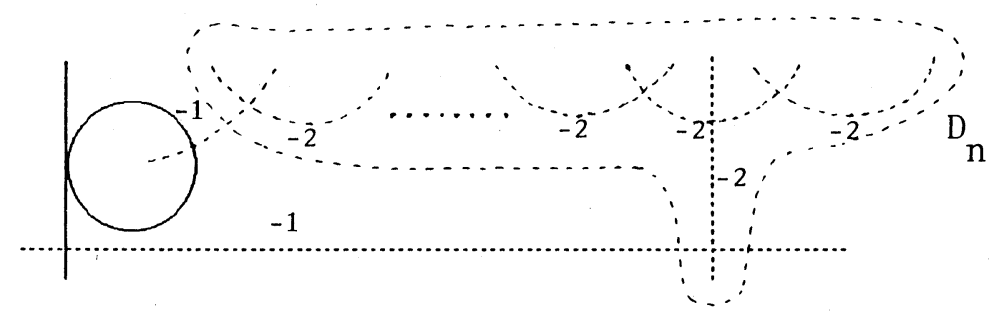

Figure $4 \quad D_{n}$-manifolds

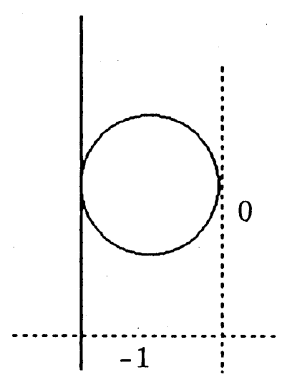

Figure 5

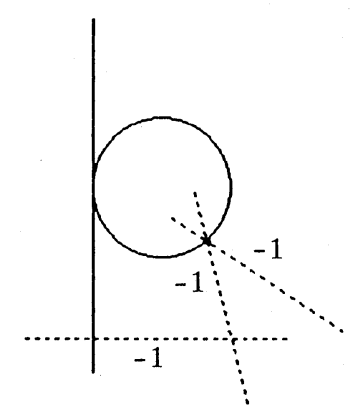

Atiyah-Hitchin manifolds

All of approximately Ricci-flat Kähler metrics defined on $A_{n}, D_{n}$, the Page-Hitchin and the Atiyah-Hitchin manifolds are modifications (see (2-47) and (2-49)) of the standard flat metric of $C^{*} \times C$. All of them are ALF. Therefore the weighted Sobolev inequality is valid on these manifolds, i.e., there exists a positive constant $C$ such that

$$
\left(\int r^{-1}|f|^{4}\right)^{\frac{1}{2}} \leq C \int|d f|^{2}
$$




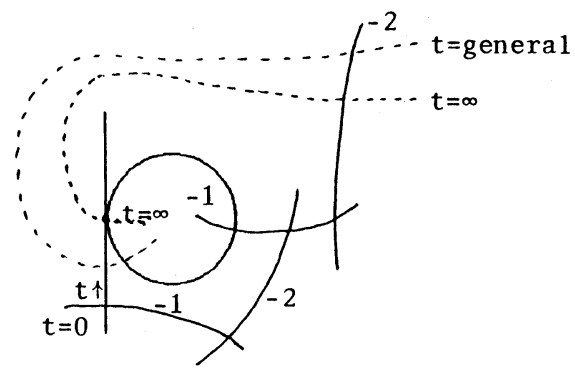

Figure $6 \quad$ coordinate $t$

holds for any $L_{; 1}^{2}$-function $f$ with compact support, where $r$ is the distance function from a fixed point. Yau $[Y]$ used Sobolev inequality and Moser's iteration technique $[\mathrm{M}]$ to derive a $C^{0}$ estimate for the solution of a Monge-Ampère equation on a compact Kähler manifold. In [B-K], Bando and Kobayashi used the Sobolev inequality for functions with compact support to get a $C^{0}$ estimate on a noncompact manifold which is asymptotically a warped product $d r^{2}+r^{2} g$. Although the Sobolev inequality does not hold for ALF spaces, we have the above weighted Sobolev inequality. It is not difficult to modify the arguments of [B$\mathrm{K}$ ] (see also [Ko2]) for these manifolds by using the weighted Sobolev inequality instead of the usual one.

We solve the Monge-Ampère equation

$$
\left(\omega_{0}+\sqrt{-1} \partial \bar{\partial} u\right)^{2}=e^{-f} \omega_{0}^{2}(=V)
$$

with

$$
f=O\left(r^{-(2+\delta)}\right)
$$

where $r$ is the distance function from a fixed point and $V$ is a canonical Ricci-flat volume form on the manifold under consideration. We assume

$$
0<\delta<1
$$

We define two Banach spaces $B_{1}$ and $B_{2}$ as follows. $B_{1}$ is the Banach space of all $C^{k, \alpha}$ functions $u$ such that $|u|=O\left(r^{-\delta}\right)$ and its derivatives decay as in the definition of $C_{\delta}^{k, \alpha}$ in [B-K] (see also [Ko2]). $B_{2}$ consists of $C^{k-2, \alpha}$ functions $f$ such that $|f|=O\left(r^{-(2+\delta)}\right)$ and its derivatives decay as in the definition of $C_{\delta+2}^{k-2, \alpha}$ in [B-K]. The reason of this choice of function spaces (the value of $\delta$ ) is that the function $\frac{1}{r}(\delta=1)$ on $R^{3}$ is a harmonic function (Green's function), i.e., $\triangle \frac{1}{r}=0$ where $\triangle$ is the 
Laplacian of the standard flat metric of $R^{3}$ and $\frac{1}{r^{6}}$ is superharmonic if $0<\delta<1$. Since an ALF metric is asymptotically $d r^{2}+r^{2} g_{1}+g_{2}$, where $g_{1}$ is a Riemannian metric of a compact surface and $g_{2}$ is the standard metric of $S^{1}$, the function $\frac{1}{r^{6}}$ is superharmonic in the asymptitic region if $0<\delta<1$, more precisely, there exists a positive constant $c$ such that

$$
\triangle \frac{1}{r^{\delta}}<c \delta(\delta-1) \frac{1}{r^{\delta}}
$$

holds if $r$ is sufficiently large. So the function $\frac{1}{r^{6}}$ plays the role of the barrier function in the sense of $[\mathrm{B}-\mathrm{K}]$. Let $O$ be the open set of $B_{1}$ consisting of those $u \in B_{1}$ for which there exists a positive number $C$ with

$$
C \omega_{0}<\omega+\sqrt{-1} \partial \bar{\partial} u<C^{-1} \omega_{0} .
$$

Then we define

$$
\Phi: O \rightarrow B_{2}, \quad u \mapsto \log \frac{\left(\omega_{0}+\sqrt{-1} \partial \bar{\partial} u\right)^{2}}{\omega_{0}^{2}} .
$$

We use the continuity method to solve $(2-51): \Phi(u)=-f$. Modifying the arguments in $[\mathrm{B}-\mathrm{K}]$, we can see that there exists a constant $C$ such that for a given $f \in B_{2}$, we can find a solution $u \in O$ for the equation $\Phi(u)=f$ with estimate

$$
\|u\|_{B_{1}} \leq C\|f\|_{B_{2}} .
$$

To show a $C^{0}$-estimate, we use the weighted Sobolev inequality. The condition $f=O\left(r^{-(2+\delta)}\right)$ with $0<\delta$ guarantees the existence of an a priori $L^{2 p}$ estimate of $u$ for some sufficiently large $p$. We can start Moser's iteration scheme $[\mathrm{M}]$ with this estimate. To show decay estimates, we use $r^{-\delta}$ as a barrier function as in [B-K].

Theorem 1. Let $X$ be compact complex surface obtained by blowing up $P_{2}(C)$ several times. Let $D$ be an anticanonical reduced divisor composed of two rational curves with one ordinary contact point and without any other intersection. If one of the components of $D$ has selfintersection number 0 or 1 , then the space $X-D$ is diffeomorphic to one of the following manifolds and admits an ALF complete Ricci-flat (hyper-)Kähler metric:

(i) $C^{2}$

(ii) $A_{i}$-manifolds $(i=1,2, \cdots)$

(iii) $D_{i}$-manifolds $(i=0,1,2, \cdots)$, where $D_{0}$ and $D_{1}$-manifolds are the Atiyah-Hitchin manifolds and $D_{2}$-manifold is the Page-Hitchin manifold. 


\subsection{Ricci-flat Kähler metrics on affine algebraic man- ifolds}

Let $\left(X^{n}, D\right)$ be a pair of a Fano manifold $X$ and a smooth hypersurface $D$ in $X$. We assume $c_{1}(X)=\alpha[D]$ with $\alpha \geq 1$. In the last section, we have constructed an approximately Ricci-flat complete Kähler metric $\omega_{0}$ on $X-D$. In this section, we prove the existence of a complete Ricci-flat Kähler metric of the form $\omega=\omega_{0}+\sqrt{-1} \partial \bar{\partial} u$ together with optimal decay estimates on $u$. To do so, we solve the Monge-Ampère equation

$$
\left(\omega_{0}+\partial \bar{\partial} u\right)^{n}=e^{-f} \omega_{0}^{n}
$$

where $f$ has decay estimate as in Lemma 6 if $\alpha>1$ or (2-21) (i.e., $|f| \approx e^{-\frac{t}{2}}$ ) if $\alpha=1$. We apply the continuity method (see [Bou] and [Y2]) to the family of Monge-Ampère equations

$$
\left(\omega_{0}+\sqrt{-1} \partial \bar{\partial} u\right)^{n}=e^{-t f} \omega_{0}^{n} . \quad t \in[0,1]
$$

We have shown in Section 2 that there exists a Ricci-flat volume form $V$ such that $f=\log \frac{V}{\omega_{0}^{n}}$ fulfills the decay conditions in Lemma 6 if $\alpha>1$ or (2-21) if $\alpha=1$.

\subsubsection{The case $\alpha>1$}

Let $O$ be an open set of a Banach space $C_{\delta}^{k, \alpha}$ defined by

$$
O=\left\{u \in C_{\delta}^{k, \alpha} ; \exists a>0 \text { with } a \omega_{0}<\omega=\omega_{0}+\sqrt{-1} \partial \bar{\partial} u<a^{-1} \omega_{0}\right\}
$$

and consider the map

$$
\Phi: O \longrightarrow C_{2+\delta}^{k, \alpha}, \quad u \mapsto \log \frac{\omega^{n}}{\omega_{0}^{n}} .
$$

Then (2-57) is equivalent to

$$
\Phi(u)=-t f .
$$

As in Yau's solution of Calabi's conjecture, we solve (2-56) by the continuity method. The Sobolev inequality (2-18) enables us to overcome the difficulty arising from noncompactness. First of all, $u=0$ is a solution at $t=0$. We want to solve (2-57) at $t=1$. It suffices to show the set

$$
\mathcal{C}=\{t \in[0,1] ;(2-57) \text { has a solution at } t\} \subset[0,1]
$$

is open and closed. 


\section{Openness.}

We show that the linearization of $\Phi$ has a bounded inverse. The linearization of $\Phi$ at $\omega=\omega_{0}+\sqrt{-1} \partial \bar{\partial} u(u \in O)$ is the Laplacian $\triangle_{\omega}$ with respect to the metric $\omega$. Since $\omega$ is asymptotically equal to $\omega_{0}$, the Banach space $C_{\delta}^{k, \alpha}$ with respect to $\omega$ is the same space as that defined from $\omega_{0}$ and two norms are equivalent. Moreover, Lemma 7 holds outside a compact set, if we consider the function $\rho$, which was defined from $\omega_{0}$, with the background metric $\omega$ instead of $\omega_{0}$. Set $\triangle=\triangle_{\omega}$. From the implicit function theorem of differentiable maps between Banach spaces, it suffices to show that there exists a positive number $c$ such that for all $g \in C_{2+\delta}^{k, \alpha}$ the equation

$$
\triangle v=g
$$

has a solution $v \in C_{\delta}^{k+2, \alpha}$ with estimate

$$
\|v\|_{C_{\delta}^{k+2, \alpha}} \leq c\|g\|_{C_{2+\delta}^{k, \alpha}}
$$

We solve (2-59) as a limit of Dirichlet problems on relatively compact domains in $X-D$. Since $g \in C_{2+\delta}^{k, \alpha}$ for a positive number $\delta$, there exists a positive number $1<q=q(\delta)<n$ such that $g \in L^{q}(X-D, \omega)$. If we set $\gamma=\frac{n}{n-1}$ and $q^{\prime}=\frac{q}{q-1}$, then we have $q^{\prime}>\gamma$. Suppose $v$ is a solution of (2-59) on some relatively compact domain with Dirichlet boundary condition. Multiplying the equation (2-59) by $-|v|^{p-2} v$ and integrating by parts (with respect to the volume form $\frac{\omega^{n}}{n !}$ ), we have

$$
\begin{aligned}
\int-|v|^{p-2} v g & =\int-|v|^{p-2} v \Delta v \\
& =(p-1) \int|v|^{p-2} \partial|v| \wedge \bar{\partial}|v| \wedge \frac{\omega^{n-1}}{(n-1) !} \\
& =\left.\left.\frac{4(p-1)}{p^{2}} \int|\partial| v\right|^{\frac{p}{2}}\right|^{2}
\end{aligned}
$$

and hence

$$
\left.\left.\int|\partial| v\right|^{\frac{p}{2}}\right|^{2}=-\frac{p^{2}}{4(p-1)} \int g|v|^{p-2} v .
$$

If $p \geq p_{-1}=\frac{q^{\prime}}{q^{\prime}-\gamma}>1$, we have from the Sobolev inequality $(2-18)$ that

$$
\left(\int|v|^{p \gamma}\right)^{\frac{1}{\gamma}}=\left(\int\left(|v|^{\frac{p}{2}}\right)^{\frac{2 n}{n-1}}\right)^{\frac{n-1}{n}} \leq C p \int|g||v|^{p-1}
$$


for some positive constant $C$ independent of $g, v, p$ and domains. Hereafter, $C$ always represents such positive constant which may differ in different occurrences. We now have an estimate for the $L^{q}$-norm of $g$. Applying the Hölder inequality to (2-61), we have

$$
\left(\int|v|^{p \gamma}\right)^{\frac{1}{\gamma}} \leq C p\left(\int|g|^{q}\right)^{\frac{1}{q}}\left(\int|v|^{q^{\prime}(p-1)}\right)^{\frac{1}{q^{\prime}}} .
$$

If $p=p_{-1}$, then $p_{-1} \gamma=\frac{q^{\prime} \gamma}{q^{\prime}-\gamma}=q^{\prime}\left(p_{-1}-1\right)$ and $\frac{1}{\gamma}-\frac{1}{q^{\prime}}=\frac{1}{p_{0}}$. Setting $p_{0}=p_{-1} \gamma$, we have

$$
\left(\int|v|^{p_{0}}\right)^{\frac{1}{p_{0}}} \leq C p_{-1}\left(\int|g|^{q}\right)^{\frac{1}{q}}
$$

and thus we have

$$
v \in L^{p_{0}}(X-D, \omega)
$$

with estimate (2-62). We write $C$ instead of $C p_{-1}$ in (2-62) from here on. Applying again the Hölder inequality to (2-61), we have

$$
\begin{aligned}
\left(\int|v|^{p \gamma}\right)^{\frac{1}{\gamma}} & \leq C p\left(\int|g|^{p}\right)^{\frac{1}{p}}\left(\int|v|^{p}\right)^{\frac{p-1}{p}} \\
& \leq C p\|g\|_{q}^{\frac{q}{p}}\|g\|_{\infty}^{\frac{p-q}{p}}\|v\|_{p}^{p-1} .
\end{aligned}
$$

Setting $p=p_{i}=p_{-1} \gamma^{i+1}=p_{0} \gamma^{i}$, we have

$$
\|v\|_{p_{i+1}} \leq\left(C p_{i}\right)^{\frac{1}{p_{i}}}\|g\|_{q}^{\frac{q}{p_{i}^{2}}}\|g\|_{\infty}^{\frac{p_{i}-q}{p_{i}^{2}}}\|v\|_{p_{i}}^{\frac{p_{i}-1}{p_{i}}} .
$$

Iterating this process, we have

$$
\|v\|_{\infty} \leq C\|g\|_{q}^{\theta}\|g\|_{\infty}^{1-\theta}
$$

with $\theta=\frac{q}{n}$. Computing the correct value of $\theta$ directly is not easy. Here, we compute this by examining the effect of a scale change of the metric. Iterating the process (2-63) and using (2-62), we have

$$
\begin{aligned}
\|v\|_{p_{i+1}} & \leq C\|g\|_{q}^{a_{i}}\|g\|_{\infty}^{b_{i}}\|v\|_{p_{0}}^{c_{i}} \quad\left(a_{i}+b_{i}+c_{i}=1\right) \\
& \leq C\|g\|_{q}^{a_{i}^{\prime}}\|g\|_{\infty}^{b_{i}} \quad\left(a_{i}^{\prime}+b_{i}=1\right) .
\end{aligned}
$$

Taking the limit $i \rightarrow \infty$, we have

$$
\|v\|_{\infty} \leq C\|g\|_{q}^{\theta}\|g\|_{\infty}^{1-\theta} \quad \text { for some } \theta
$$


The scale change $\omega \rightarrow \alpha \omega$ transforms the equation (2-59) to

$$
\triangle_{\alpha \omega}(\alpha v)=g
$$

with the estimate

$$
\|\alpha v\|_{\infty} \leq C\|g\|_{q}^{\theta}\|g\|_{\infty}^{1-\theta} .
$$

Comparing the exponents of $\alpha$ in both sides, we have

$$
\alpha=\alpha^{\frac{n \theta}{q}} \quad \text { and hence } \theta=\frac{q}{n} \text {. }
$$

We thus get a global a priori $C^{0}$ estimate (2-64) for $u$. To get its decay estimates, we refer to Lemma 7. Since $g \in C_{2+\delta}^{k, \alpha}$, we have from Lemma 7 that outside a compact set $K$

$$
\triangle \rho^{-\delta} \leq-c|g|
$$

for some positive constant $c$, provided $\delta$ is small. Hence if $A>0$ is sufficiently large, we have

$$
\begin{aligned}
& v+A \rho^{-\delta}>0 \\
& v-A \rho^{-\delta}<0
\end{aligned}
$$

on the compact set $K$ and

$$
\begin{aligned}
& \triangle\left(v+A \rho^{-\delta}\right) \leq(1-A c)|g| \leq 0 \\
& \triangle\left(v-A \rho^{-\delta}\right) \geq(1+A c)|g| \geq 0
\end{aligned}
$$

outside of $K$. The constant $A$ depends on the maximum value of $|g|$. It follows from the classical maximum principle, (2-64), (2-65) and (2-66) that for the Dirichlet solutions of (2-59) on all sufficiently large relatively compact subdomains, we have

$$
|v| \leq A \rho^{-\delta}
$$

This gives a $C_{\delta}^{0}$-estimate for the solution $v$ of (2-59). The interior Schauder estimates [Gil-T, Chapter 6] (cf. Lemma 5) then gives a $C_{\delta}^{k, \alpha}$ bound for $v$ :

$$
\|v\|_{C_{\delta}^{k+2, \alpha}} \leq C\|g\|_{C_{2+\delta}^{k, \alpha}}
$$

Thus $\triangle: C_{\delta}^{k+2, \alpha} \rightarrow C_{2+\delta}^{k, \alpha}$ has a bounded inverse with estimate (2-68). 
Closedness.

Let $u \in C_{\delta}^{k+2, \alpha}$ and $\omega=\omega_{0}+\sqrt{-1} \partial \bar{\partial} u$. We assume that $u$ fulfills the equation (46)

$$
\omega^{n}=e^{-t f} \omega_{0}^{n}
$$

and there exists a positive number $a$ such that

$$
a \omega_{0}<\omega<a^{-1} \omega_{0} .
$$

If we get $C_{\delta}^{k+2, \alpha}$-estimates independent of $t \in[0,1]$ for all $k \in N$ and $\alpha \in(0,1)$, we are done because of Ascoli-Arzelà's Theorem. To get a $C_{\delta}^{0}$-estimate, we argue as in the proof of the openness. Multiplying the following equality

$$
\begin{aligned}
\left(1-e^{-t f}\right) \omega_{0}^{n} & =\omega_{0}^{n}-\omega^{n} \\
& =(-\partial \bar{\partial} u) \wedge\left(\omega_{0}^{n-1}+\omega_{0}^{n-2} \omega+\cdots+\omega^{n-1}\right)
\end{aligned}
$$

by $|u|^{p-2} u\left(p \geq p_{-1}\right)$ and integrating by parts, we have

$$
\begin{aligned}
& \int\left(1-e^{-t f}\right)|u|^{p-2} u \omega_{0}^{n} \\
= & \int-|u|^{p-2} u \sqrt{-1} \partial \bar{\partial} u \wedge\left(\omega_{0}^{n-1}+\omega_{0}^{n-2} \omega+\cdots+\omega^{n-1}\right) \\
= & \frac{4(p-1)}{p^{2}} \int \sqrt{-1} \partial|u|^{\frac{p}{2}} \wedge \bar{\partial}|u|^{\frac{p}{2}}\left(\omega_{0}^{n-1}+\omega_{0}^{n-2} \omega+\cdots+\omega^{n-1}\right) \\
\geq & \left.\left.\frac{4(p-1)}{p^{2}} \int|\partial| u\right|^{\frac{p}{2}}\right|^{2} \omega_{0}^{n} .
\end{aligned}
$$

Using the Sobolev inequality (2-18) as before, we have

$$
\left(\int|u|^{p \gamma}\right)^{\frac{1}{\gamma}} \leq C p \int|f||u|^{p-1}
$$

where we have used the volume form $\omega_{0}^{n}$ and the Sobolev inequality (218) with respect to $\omega_{0}$. Hereafter we use $\omega_{0}$ as a background metric. Since (2-70) is formally the same as $(2-61)$, we conclude as before the following:

$$
\|u\|_{\infty} \leq C\|f\|_{q}^{\theta}\|f\|_{\infty}^{1-\theta} \quad \text { with } \theta=\frac{q}{n} .
$$

So far we have ignored the boundary terms in integration by parts because of its small contribution. More precisely, we should argue as follows: 
Let $\phi_{R}$ be a cut-off function such that $\phi_{R}=1$ on $B(R)$ and $\phi_{R}=0$ outside of $B(2 R)$. We consider the equation

$$
\left(\omega_{0}+\sqrt{-1} \partial \bar{\partial}\left(\phi_{R} u\right)\right)^{n}=e^{f_{R}} \omega_{0}^{n}
$$

where $u$ is the solution we started with and $f_{R}=f$ on $B(R), f_{R}=0$ outside of $B(2 R)$. Since $u \in C_{\delta}^{k+2, \alpha}$, we see that $\omega_{0}+\sqrt{-1} \partial \bar{\partial}\left(\phi_{R} u\right)>0$ for sufficiently large $R$. By the same argument as in the proof of the openness, we have

$$
\left\|\phi_{R} u\right\|_{\infty} \leq C\left\|f_{R}\right\|_{q}^{\theta}\|f\|_{\infty}^{1-\theta} .
$$

Letting $R \rightarrow \infty$, we conclude

$$
\|u\|_{\infty} \leq C\|f\|_{q}^{\theta}\|f\|_{\infty}^{1-\theta} \quad \text { with } \theta=\frac{q}{n} .
$$

For the decay estimate, we use (iii) of Lemma 7. Let $K$ be a sufficiently large positive number such that $-K \rho^{-\delta}<u<K \rho^{-\delta}$ on a compact set and $-c K \rho^{-2-\delta}<f<c K \rho^{-2-\delta}$ on $X-D$, where $c$ is as in Lemma 7. Such $K$ exists because of the $C^{0}$-estimate (58) of $u$ and the decay estimate of $f \in C_{2+\delta}^{k, \alpha}$. Set $\left.\widetilde{\omega}_{0}=\omega_{0}+\sqrt{-1} \partial \partial \bar{\partial} K \rho^{-\delta}\right)$. Then Lemma 7 implies $\widetilde{\omega}_{0}^{n} \leq\left(1-c K \rho^{-2-\delta}\right) \widetilde{\omega}_{0}^{n}$. For such $K$, we compute

$$
\begin{aligned}
& n \log \left(1+\frac{\triangle_{\tilde{\omega}_{0}}\left(u-K \rho^{-\delta}\right)}{n}\right) \\
\geq & \log \frac{\left(\widetilde{\omega}_{0}+\sqrt{-1} \partial \bar{\partial}\left(u-K \rho^{-\delta}\right)\right)^{n}}{\widetilde{\omega}_{0}^{n}}=\log \frac{\omega^{n}}{\widetilde{\omega}_{0}^{n}} \\
\geq & -f-\log \left(1-c K \rho^{-2-\delta}\right) \geq-f+c K \rho^{-2-\delta} \geq 0
\end{aligned}
$$

if $\rho$ is sufficiently large, say $\rho>N$. Hence we have

$$
\triangle_{\tilde{\omega}_{0}}\left(u-K \rho^{-\delta}\right) \geq 0
$$

for $\rho>N$. We may assume $u-K \rho^{-\delta}<0$ on a compact set $\rho \leq 2 N$. And at infinity we have $u-K \rho^{-\delta} \rightarrow 0$. By the classical maximum principle for subharmonic functions, we have

$$
u-K \rho^{-\delta} \leq 0
$$

outside of a compact set. Similarly, we have

$$
u+K \rho^{-\delta} \geq 0
$$


outside of a compact set. We thus have a $C_{\delta}^{0}$-estimate for $u$ :

$$
\|u\|_{C_{\delta}^{0}} \leq C\|f\|_{q}^{\theta}\|f\|_{\infty}^{1-\theta} \quad \text { with } \theta=\frac{q}{n} .
$$

We now proceed to get a $C_{\delta}^{2}$-bound. To get an ordinary $C^{2}$-estimate for $u$, we need the Chern-Lu formula (Proposition 11): (cf. Yau's Schwarz lemma [Y3]):

Lemma 13. Let $(M, g)$ be a Kähler manifold, $(N, h)$ a Hermitian manifold and $f: M \rightarrow N$ be a holomorphic mapping. Set $u=\|\partial f\|^{2}$. Then we have

$$
\triangle \log u \geq \frac{\operatorname{Ric}_{g}(\partial f, \overline{\partial f})}{\|\partial f\|^{2}}-\frac{\operatorname{Bisect}_{h}(\partial f, \overline{\partial f}, \partial f, \overline{\partial f})}{\|\partial f\|^{2}} .
$$

Proof. We get the desired inequality if we combine the Chern$\mathrm{Lu}$ formula with the following Schwarz-type inequality for holomorphic mappings:

$$
\|\partial\| \partial f\left\|^{2}\right\|^{2} \leq\left\|D^{\prime} \partial f\right\|^{2}\|\partial f\|^{2} \text {. }
$$

This inequality can be seen in the following way. We equip $T^{(1,0)} M$ and $T^{(1,0)} N$ with the Hermitian connections (the Kähler condition is that the Hermitian connection agrees with the Levi-Civita connection.). Then we have the Hermitian connection on $T^{*(1,0)} M \otimes f^{-1} T^{(1,0)} N$. Using this connection, we compute

$$
\begin{aligned}
& \|\partial\| \partial f\left\|^{2}\right\|^{2}=\left\|\left(g \otimes f^{-1} h\right)\left(D^{\prime} \partial f, \partial f\right)\right\|^{2} \\
& \quad\left(\text { since } D^{\prime \prime}=\bar{\partial} \text { and } f \text { is holomorphic }\right) \\
& \leq\left\|D^{\prime} \partial f\right\|^{2}\|\partial f\|^{2} .
\end{aligned}
$$

We use Lemma 13 to $i d:(X-D, \omega) \rightarrow\left(X-D, \omega_{0}\right)$. We note that

$$
\|\partial f\|^{2}=\operatorname{tr}_{\omega} \omega_{0} .
$$

Since $\|\operatorname{Ric}(\omega)\|=(1-t)\left\|\operatorname{Ric}\left(\omega_{0}\right)\right\|_{\omega_{0}}$ and $\left\|\operatorname{Bisect}_{\omega_{0}}\right\|$ is bounded, we have from Lemma 11 that

$$
\triangle \log \operatorname{tr}_{\omega} \omega_{0} \geq-C_{1}-C_{2} \operatorname{tr}_{\omega} \omega_{0}
$$

where $\triangle=\triangle_{\omega}$ and $C_{i}$ 's are positive constants depending only on the initial metric $\omega_{0}$. Since

$$
\triangle u=n-\operatorname{tr}_{\omega} \omega_{0}
$$


we have

$$
\triangle\left(\log \operatorname{tr}_{\omega} \omega_{0}-A u\right) \geq-A n+\left(A-C_{2}\right) \operatorname{tr}_{\omega} \omega_{0}-C_{1} .
$$

We choose $A$ so that $A-C_{2}>0$. Since $\log \operatorname{tr}_{\omega} \omega_{0}-A u$ is bounded by assumption, we can use the following Yau's maximum principle [Y1]:

Fact 2 (Yau). Let $M$ be a complete Riemannian manifold with Ricci curvature bounded from below. Let $f$ be a $C^{2}$ function which is bounded from above on $M$. Then for all $\varepsilon>0$, there exists a point $p$ in $M$ such that at $p$,

$$
\|d f\|<\varepsilon, \Delta f<\varepsilon \text { and } f(p)>\sup f-\varepsilon .
$$

Since $A-C_{2}>0$, we deduce from (2-74) and (2-75) that for all $\varepsilon>0$ there exists a point $p$ in $X-D$ such that

$$
\begin{aligned}
\log \operatorname{tr}_{\omega} \omega_{0}(p)-A u(p) & >\sup \left(\log \operatorname{tr}_{\omega} \omega_{0}-A u\right)-\varepsilon \\
\operatorname{tr}_{\omega} \omega_{0}(p) & <\frac{\varepsilon+A n+C_{1}}{A-C_{2}} .
\end{aligned}
$$

Letting $\varepsilon \rightarrow 0$, we get

$$
\sup \operatorname{tr}_{\omega} \omega_{0}<\frac{A n+C_{1}}{A-C_{2}} e^{A(\sup u-\inf u)}=C_{3} .
$$

Here, we have used the a priori $C^{0}$ estimate of $u$. This implies $\omega_{0}<$ $C_{3} \omega$. Combining this with the equation (2-56), we have an ordinary $C^{2}$-estimate of $u$ :

$$
a \omega_{0}<\omega<a^{-1} \omega_{0}
$$

for some positive constant $a$ depending only on $\omega_{0}$. We now recall the interior Hölder estimates for second derivatives of solutions of certain fully non-linear elliptic equations: [Gil-T, Theorem 17.14], which we can apply to get a $C^{2, \alpha}$-estimate for $u$ in terms of the ordinary $C^{2}$-estimate (2-76) of $u$ :

$$
\|u\|_{C^{2, \alpha}}<C
$$

where $C$ is a positive constant depending only on $\omega_{0}$. Suppose there exists a solution for $t \in\left[0, t_{0}\right) \subset[0,1]$. Differentiating the equation

$$
\log \frac{\omega^{n}}{\omega_{0}^{n}}=-t f
$$


with respect to $t$ (at $t<t_{0}$ ), we get

$$
\triangle_{\omega} \frac{\partial u}{\partial t}=-f
$$

From (2-76) the Banach spaces $C_{\delta}^{k, \alpha}$ are independent of $t<t_{0}$. Since $f \in C_{2+\delta}^{k, \alpha}$, we first get an a priori $C_{\delta}^{2, \alpha}$-estimate for $\frac{\partial u}{\partial t}$ independent of $t<t_{0}$. We thus get an a priori $C_{\delta}^{2, \alpha}$-estimate of $u$. Expressing the equation (2-58) in local coordinates

$$
\log \frac{\operatorname{det}\left(g_{i \bar{j}}+u_{i \bar{j}}\right)}{\operatorname{det}\left(g_{i \bar{j}}\right)}=-t f
$$

and differentiating this with respect to $z^{k}$, we get

$$
g^{i \bar{j}} u_{k, i \bar{j}}=\left(-g^{\prime i \bar{j}}+g^{i \bar{j}}\right) g_{i \bar{j}, k}-t f_{k}
$$

where $g_{i \bar{j}}^{\prime}=g_{i \bar{j}}+u_{i \bar{j}}$. Applying the interior Schauder estimates and the $C_{\delta}^{2, \alpha}$-estimate of $u$, we get a priori $C_{\delta}^{l+2, \alpha}$-estimates of $u$ for $l \leq k$ by the bootstrapping argument. We thus get an a priori $C_{\delta}^{k+2, \alpha}$-estimate of $u$ which is independent of $t$.

Another way to get a $C_{\delta}^{k, \alpha}$-estimate for $u$ is as follows. Consider a local coordinate system around a point $p$ with $\operatorname{dist}(o, p)=r$ which appeared in the definition of asymptotically flat geometry. Define

$$
F(x, \partial \bar{\partial} v)=\log \frac{\left(\frac{1}{r^{2}} \omega_{0}+\sqrt{-1} \partial \bar{\partial} v\right)^{n}}{\left(\frac{1}{r^{2}} \omega_{0}\right)^{n}}
$$

If we set $v=r^{\delta} u$ and $h=r^{-2-\delta}$, then the equation (2-56) is written as

$$
\frac{1}{h} F(x, h \partial \bar{\partial} v)=-\frac{1}{h}(t f)=-t\left(\frac{-f}{h}\right) .
$$

Since we already have an a priori $C^{2}$-estimate for $u$ and $F(x, 0)=0$, the above equation fulfills the conditions of [Gil-T, Theorem 17.15] with respect to $x$ and $\partial \bar{\partial} v$. Now $\frac{f}{h}$ is estimated, because $f \in C_{2+\delta}^{k+2, \alpha}$. Thus, from the a priori $C_{\delta}^{0}$-estimate (2-73), we have a priori $C^{2, \alpha}$-estimates for $v$. These are nothing but $C_{\delta}^{2, \alpha}$-estimates for $u$.

This completes the proof of the existence of a complete Ricci-flat Kähler metric on $X-D$ with $\alpha>1$. We sum up the arguments in the following theorems. 
Theorem 2. Let $(X, D)$ be a pair of a Fano manifold $X$ and a smooth hypersurface $D$ in $X$ such that $c_{1}(X)=\alpha[D]$ with $\alpha>1$. Assume that $D$ admits a Kähler-Einstein metric. Then there exists a complete Ricci-flat Kähler metric on $X-D$.

Theorem 3. Let $\omega_{0}$ be a complete Kähler metric on an $n$ dimensional $(n \geq 2)$ noncompact complex manifold $Y$. Assume that $\omega_{0}$ has $C^{k, \alpha}$-asymptotically flat geometry with $k \geq 2$ and $0<\alpha<1$ and the volume of the geodesic ball grows like $d^{2 n}$. Assume moreover that there exists a function $\rho$ which fulfills the properties of Lemma 4, (1) and Lemma 7, outside of a compact set. If $Y$ admits a Ricci-flat volume form $V$ such that $\omega_{0}^{n}=e^{f} V$ with $f \in C_{2+\delta}^{k, \alpha}$ and $\delta>0$, then there exists a $C_{\delta}^{k+2, \alpha}$-bounded function $u$ such that $\omega_{0}+\sqrt{-1} \partial \bar{\partial} u$ is a complete Ricci-flat Kähler metric.

Recently Nadel [N], Siu [S1], Tian [T1], [T2] and Tian-Tau [T-Y] proved the existence of a Kähler-Einstein metric on the Fermat hypersurfaces $F$ of degrees $n-1$ and $n$ in $P_{n}(C)$.

Example 1. Let $F$ be a smooth hyperquadric or a Fermat hypersurface of degree $n$ or $n-1$ in $P_{n}(C)$. Then the space $P_{n}(C)-F$ admits a complete Ricci-flat Kähler metric.

The existence of a complete hyper-Kähler metric on the minimal resolutions $Y$ of the Kleinian singularities $C^{2} / \Gamma$ is a direct consequence of Theorem 3, where $\Gamma$ is a finite subgroup of $S U(2)$. It follows from the remark just after Lemma 7 that the metric is ALE with the curvature decay $O\left(r^{-6}\right)$. Next we consider the Milnor fiber $[\mathrm{Kr} 1]$ of $C^{2} / \Gamma$. The compactified factor space $P_{2}(C) / \Gamma$ has quotient singularities at the origin and at some points on the image $D$ of the line at infinity. We regard the image $D$ as an orbi-curve with the Fubini-Study metric. Since a Milnor fiber $Y^{\prime}$ has the same boundary curve, we can apply the orbifold version of the proof of Theorem 2. We thus see that the Milnor fiber $Y^{\prime}$ admits a complete hyper-Kähler metric. Since the curvature is $L^{2}$ integrable, the metric is in fact ALE with curvature decay $O\left(r^{-6}\right)$ (see [BKN] or Nakajima's survey in this volume.). So, we get an analytical interpretation of a part of Kronheimer's result $[\mathrm{Kr} 1,2]$ :

Theorem 4 (Kronheimer). Let $Y^{\prime}$ be a Milnor fiber of a Kleinian singularity $C^{2} / \Gamma$. Then $Y^{\prime}$ admits a complete hyper-Kähler ALE metric.

Remark. Kronheimer $[\mathrm{Kr} 1,2]$ classified all ALE gravitational instantons. These are the minimal resolutions of the Kleinian singularities and their Milnor fibers. 


\subsubsection{Concentrating Ricci curvature at infinity}

Let $(X, D)$ be as in 2.3.1, namely $X$ is a Fano manifold of dimension $n \geq 2$ and $D$ is a smooth hypersurface admitting a Kähler-Einstein metric such that $c_{1}(X)=\alpha[D]$ with $\alpha>1$. In view of Proposition 9, we see that there exists a sequence $\gamma_{i}$ of real closed nonnegative $(1,1)$ forms in $2 \pi c_{1}(X)$ such that $\gamma_{i} \rightarrow 0$ outside of $D$ and converges to $\alpha D$ as currents (cf. [Gri-Har, Chapter 3]). We now recall Yau's solution of Calabi's conjecture [Y2]:

Fact 3 ([Y2]). Let $M$ be a compact Kähler manifold and $\gamma$ a real closed $(1,1)$-form in $2 \pi c_{1}(M)$. Then in each Kähler class of $M$ there exists a unique Kähler form with $\gamma$ as its Ricci form.

Positive multiples of $c_{1}(X)$ are Kähler classes of $X$. We can thus solve the problem of finding a Kähler metric $\omega_{i}$ in a certain positive multiple of $c_{1}(X)$ with a prescribed Ricci form $\gamma_{i}$. This is a process of concentrating Ricci curvature at infinity. If we take the limit $i \rightarrow \infty$, we might reach a limit Ricci-flat Kähler metric on $X-D$. In this subsection, we give an alternative proof of Theorem 2 by showing that the sequence $\omega_{i}$ in fact converges to a Ricci-flat Kähler metric on $X-D$.

Let $t, \theta, V, f$ and $\omega_{0}$ be as in Section 2.2.1 and suppose that the conditions of Lemma 3 are fulfilled. We introduce a small positive number $\varepsilon$. Solving the equation

$$
\operatorname{Ric}\left(\widetilde{\omega}_{\varepsilon}\right)=-\sqrt{-1} \partial \bar{\partial} \log \left(\frac{\|\sigma\|^{2}}{\|\sigma\|^{2}+\varepsilon}\right)^{\alpha}, \quad\left[\omega_{\varepsilon}\right] \propto c_{1}(X)
$$

is (up to scale changes) equivalent to finding a Kähler metric in a positive multiple of $c_{1}(X)$ with a prescribed volume form

$$
V_{\varepsilon}=\left(\frac{\|\sigma\|^{2}}{\|\sigma\|^{2}+\varepsilon}\right)^{\alpha} V
$$

Set

$$
\begin{aligned}
\omega_{\varepsilon} & =\sqrt{-1} \partial \bar{\partial} G_{\varepsilon}(t) \\
& =g_{\varepsilon}(t) \theta+g_{\varepsilon}^{\prime}(t) \sqrt{-1} \partial t \wedge \bar{\partial} t \\
& =\frac{1}{\left(e^{-t}+\varepsilon\right)^{\frac{\alpha-1}{n}}}\left\{\theta+\frac{(\alpha-1)}{n}\left(\frac{e^{-t}}{e^{-t}+\varepsilon}\right) \sqrt{-1} \partial t \wedge \bar{\partial} t\right\}
\end{aligned}
$$

where

$$
\begin{aligned}
& g_{\varepsilon}=\frac{1}{\left(e^{-t}+\varepsilon\right)^{\frac{\alpha-1}{n}}} \\
& g_{\varepsilon}=G_{\varepsilon}^{\prime}(t) .
\end{aligned}
$$


Then $\omega_{\varepsilon}$ is a Kähler metric on $X$ and satisfies

$$
\begin{aligned}
{\left[\omega_{\varepsilon}\right] } & \propto c_{1}(X) \\
\lim _{\varepsilon \rightarrow 0} \omega_{\varepsilon} & =\omega_{0} \quad \text { on } \quad X-D .
\end{aligned}
$$

By taking a scale change of $V_{\varepsilon}$ if necessary, we may assume

$$
\int V_{\varepsilon}=\int \omega_{\varepsilon}^{n}
$$

We define a function $f_{\varepsilon}$ by

$$
f_{\varepsilon}=\log \frac{\omega_{\varepsilon}^{n}}{V_{\varepsilon}} .
$$

Since $D$ admits a Kähler-Einstein metric $\theta_{D}$, we have

$$
f_{\varepsilon}=f+O(\varepsilon)
$$

From this we infer that there exists a positive number $q$ with $\alpha-1<$ $q<n$ such that

$$
\left\|f_{\varepsilon}\right\|_{L^{q}\left(X, \omega_{\varepsilon}\right)}<\text { a constant independent of } \varepsilon .
$$

By [Y2], there exists a unique elliptic solution to the equation

$$
\left(\omega_{\varepsilon}+\sqrt{-1} \partial \bar{\partial} u_{\varepsilon}\right)^{n}=V_{\varepsilon}=e^{-f_{\varepsilon}} \omega_{\varepsilon}^{n}
$$

with the normalization condition

$$
\int u_{\varepsilon} \omega_{\varepsilon}^{n}=0
$$

Thus the prescribed Ricci form $\gamma_{\varepsilon}$ of the Kähler metric $\widetilde{\omega}_{\varepsilon}=\omega_{\varepsilon}+$ $\sqrt{-1} \partial \bar{\partial} u_{\varepsilon}$ is

$$
\gamma_{\varepsilon}=\alpha \varepsilon\left(\frac{1}{\|\sigma\|^{2}+\varepsilon} \theta+\frac{1}{\|\sigma\|^{2}\left(\|\sigma\|^{2}+\varepsilon\right)^{2}} \partial\|\sigma\|^{2} \wedge \bar{\partial}\|\sigma\|^{2}\right)>0
$$

which concentrates along $D$ (at infinity) in the limit $\varepsilon \rightarrow 0$.

Gallot's isoperimetric inequality [Ga] implies that the Sobolev constant $c$ in (2-17) for functions with $\int f=0$ on a compact Riemannian manifold $(M, g)$ of dimension $n$ is bounded below by

$$
\frac{V(g)^{\frac{1}{n}}}{D(g)} G(n, D(g) \rho(g))
$$


where $V(g), D(g)$ and $\rho(g)$ stand for the volume, diameter and the lower bound of the Ricci curvature of $g$. A direct computation shows that the quantity $V^{\frac{1}{2 n}} / D$ is uniformly bounded for the family of Kähler metrics $\omega_{\varepsilon}$. So the Sobolev constants in (2-17) for functions $f$ with $\int f=0$ are uniformly bounded for the family of compact Kähler manifolds $\left(X, \omega_{\varepsilon}\right)$. In particular, there exists a positive number $c$ such that the Sobolev inequality $(2-18)$

$$
c\left(\int|f|^{\frac{2 n}{n-1}}\right)^{\frac{n-1}{n}} \leq \int|d f|^{2}
$$

for $C^{1}$-functions with $\int f \omega_{\varepsilon}^{n}=0$ holds for all $\left(X, \omega_{\varepsilon}\right)$.

Since, for some $q<n$, there is a $L^{q}\left(X, \omega_{\varepsilon}\right)$-bound (2-61) for $f_{\varepsilon}$ independent of $\varepsilon$, the same argument as in the proof of Theorem 2 (cf. "Closedness" part) gives us an a priori $C^{0}$-estimate for the solution $u_{\varepsilon}$ of (2-62) and (2-63) which is independent of $\varepsilon$. We then get $C \omega_{\varepsilon}<\widetilde{\omega}_{\varepsilon}<$ $C^{-1} \omega_{\varepsilon}$ for a positive constant $C$ independent of $\varepsilon$ by the same argument used in deriving an ordinary $C^{2}$-estimate in the proof of Theorem 2 . The Hölder estimates for second derivatives [Gil-T, Theorem 17.14] and the interior Schauder estimates imply that we get a smooth function $u_{0}=\lim _{\varepsilon \rightarrow 0} u_{\varepsilon}$ on $X-D$. If we further apply the arguments in the proof of Theorem 2 , we get the decay estimates for $u_{0}=\lim _{\varepsilon \rightarrow 0} u_{\varepsilon}$. This completes an alternative proof of Theorem 2 .

Theorem 2 corresponds to the limit of the procedure of concentrating whole Ricci form at infinity. It would then be natural to ask what occurs in the limit of concentrating only a part of Ricci form at infinity. To describe this process, we introduce a Kähler metric $\omega_{\varepsilon, \delta}$ on $X$ which is defined by replacing $\alpha$ by $\alpha-\delta$ in the definition of $\omega_{\varepsilon}$, where $\delta$ is a small positive number such that $\alpha-\delta>1$. Then we solve the Monge-Ampère equation

$$
\left(\omega_{\varepsilon, \delta}+\sqrt{-1} \partial \bar{\partial} u_{\varepsilon, \delta}\right)^{n}=\frac{\|\sigma\|^{2 \alpha}}{\left(\|\sigma\|^{2}+\varepsilon\right)^{\alpha-\delta}} V,
$$

where the Ricci form of the left side is given by

$$
\begin{gathered}
\gamma_{\varepsilon, \delta}=\varepsilon\left(\frac{\alpha}{\|\sigma\|^{2}+\varepsilon} \theta+\frac{\alpha-\delta}{\|\sigma\|^{2}\left(\|\sigma\|^{2}+\varepsilon\right)^{2}} \partial\|\sigma\|^{2} \wedge \bar{\partial}\|\sigma\|^{2}\right) \\
+\frac{\delta\|\sigma\|^{2}}{\|\sigma\|^{2}+\varepsilon} \theta>0
\end{gathered}
$$

Set $\tilde{\omega}_{\varepsilon, \delta}=\omega_{\varepsilon, \delta}+\sqrt{-1} \partial \bar{\partial} u_{\varepsilon, \delta}$. Arguing exactly in the same way as above, we take the limit $\varepsilon \rightarrow 0$ to get a complete Kähler metric $\widetilde{\omega}_{0, \delta}=$ 
$\lim _{\varepsilon \rightarrow 0} \widetilde{\omega}_{\varepsilon, \delta}$ on $X-D$ with $\delta \theta>0$ as its Ricci form. Clearly the complete Kähler manifold $\left(X-D, \widetilde{\omega}_{0, \delta}\right)$ has $C^{k, \alpha}$-asymptotically flat geometry for any admissible $(k, \alpha)$.

Of course the complete Kähler metric $\widetilde{\omega}_{0, \delta}$ is also obtained by solving

$$
\left(\omega_{0, \delta}+\sqrt{-1} \partial \bar{\partial} u\right)^{n}=\|\sigma\|^{2 \delta} V
$$

where the background metric $\omega_{0, \delta}=\lim _{\varepsilon \rightarrow 0} \omega_{\varepsilon, \delta}$ is a complete Kähler metric with Kähler potential

$$
\exp \left(\frac{(\alpha-\delta-1) t}{n}\right)
$$

Taking the limit $\delta \rightarrow 0$ in the above process, we again get a complete Ricci-flat Kähler metric in Theorem 2. We sum up the above arguments in the following Theorem.

Theorem 5. Let $(X, D), \alpha$ be as in Theorem 2 and $\theta$ a curvature form of $L_{D}$ which is positive definite everywhere on $X$ and the restriction $\theta_{D}$ to $D$ is a Kähler-Einstein metric. Suppose $\delta$ is a nonnegative number with $\alpha-\delta>1$. Then there is a smooth family of asymptotically flat complete Kähler metrics $\widetilde{\omega}_{\delta}$ on $X-D$ with positive Ricci-form $\delta \theta$ for $\delta>0$ and Ricci-flat for $\delta=0$.

Note. Recently Yeung [Ye] constructed more general examples of complete Ricci positive Kähler metrics on affine algebraic manifolds by a similar method of [B-K]. Yeung's theorem gives examples of Ricci positively curved complete Kähler manifolds treated recently by Mok [Mok].

\subsubsection{The Case $\alpha=1$ and a geometric picture of de- generation of Kähler-Einstein metrics of K3 sur- faces}

Throughout this section, we work on the complete Kähler manifold $\left(X-D, \omega_{0}\right)$ where $X$ is a compact complex manifold with positive first Chern class, $D$ is a smooth hypersurface with $c_{1}(X)=[D]$ and $\omega_{0}$ is a complete Kähler metric (2-19) on $X-D$ arising from Calabi's construction. We assume that $f=\log \frac{V}{\omega_{0}^{n}}$ satisfies $(2-21)$ and so $f \in C_{\frac{1}{2}, 0}^{k, \alpha}$ for any admissible $(k, \alpha)$. Let $\delta$ be a sufficiently large positive number. Let $O_{\delta}^{k, \alpha}$ be an open set of a Banach space $C_{\delta}^{k, \alpha}$ defined by

$$
O_{\delta}^{k, \alpha}=\left\{u \in C_{\delta}^{k, \alpha} ; \exists a>0 \text { with } a \omega_{0}<\omega=\omega_{0}+\sqrt{-1} \partial \bar{\partial} u<a^{-1} \omega_{0}\right\}
$$


and consider the map

$$
\Phi: O_{\delta}^{k, \alpha} \longrightarrow C_{\delta+\frac{1}{n}}^{k-2, \alpha}, \quad u \mapsto \log \frac{\omega^{n}}{\omega_{0}^{n}}
$$

If $u \in C_{\delta}^{k, \alpha}$ for sufficiently large $\delta$, then $\Phi(u)$ is contained in a "hypersurface" in $C_{\delta+\frac{1}{n}}^{k-2, \alpha}$ defined by a single equation

$$
\int_{X-D}\left(e^{\Phi(u)}-1\right) \omega_{0}^{n}=0
$$

The tangent space at $\Phi(u)$ is a closed subspace of $C_{\delta+\frac{1}{n}}^{k-2, \alpha}$ given by an equation

$$
\int_{X-D} g e^{\Phi(u)} \omega_{0}^{n}=0
$$

which is equivalent to

$$
\int_{X-D} g \omega^{n}=0
$$

We want to solve the Monge-Ampère equation with integrability conditions:

$$
\begin{aligned}
\Phi(u) & =-f \\
\int_{X-D}\left(e^{-f}-1\right) \omega_{0}^{n} & =0 \\
\int_{B(R)}\left(e^{-f}-1\right) \omega_{0}^{n} & =O\left(e^{-R^{\frac{2 n}{n+1}} / 2}\right)
\end{aligned}
$$

in $O_{\delta}^{k, \alpha}$ for sufficiently large $\delta$ and any admissible $(k, \alpha)$. Note that $f \in C_{\frac{1}{2}, 0}^{k-2, \alpha} \subset C_{\delta}^{k-2, \alpha}$. The decay estimate for $\int_{B(R)}\left(e^{-f}-1\right) \omega_{0}^{n}$ follows from the definition of $f$ and (2-21).

From here on we assume that $f$ is an arbitrary function in $C_{\frac{1}{2}, 0}^{k-2, \alpha}$ which satisfies the integrability conditions in (2-82).

We want to show

$$
C_{\frac{1}{2}, 0}^{k-2, \alpha} \subset \Phi\left(C_{\delta}^{k, \alpha}\right)
$$

for any sufficiently large $\delta>0$. We show this using the continuity method as in subsection 2.3.1., namely we consider the family of equa- 
tions with integrability conditions:

$$
\begin{aligned}
\Phi(u) & =\log \left(1+\tau\left(e^{-f}-1\right)\right) \\
\int_{X-D}\left(e^{-f}-1\right) \omega_{0}^{n} & =0 \\
\int_{B(R)}\left(e^{-f}-1\right) \omega_{0}^{n} & =O\left(e^{-R^{\frac{2 n}{n+1}} / 2}\right)
\end{aligned}
$$

for $\tau \in[0,1]$ and set

$$
\mathcal{C}=\left\{\tau \in[0,1] ;(2-83) \text { has a solution in } O_{\delta}^{k, \alpha}\right\} .
$$

Clearly $0 \in \mathcal{C}$. To show $1 \in \mathcal{C}$, it suffices to show that $\mathcal{C}$ is open and closed. We first consider the openness.

\section{Openness.}

To prove the openness, it suffices to show that the linearlization of $\Phi$ has a bounded inverse. The linearlization of $\Phi$ at $u \in O_{\delta}^{k, \alpha}$ is the Laplace operator $\triangle_{\omega}$ with respect to the metric $\omega$. Since $\omega$ is asymptotically equal to $\omega_{0}$, the Banach space $C_{\delta}^{k, \alpha}$ defined from $\omega$ is the same as that defined from $\omega_{0}$ and two norms are equivalent. Set $\triangle=\triangle_{\omega}$. It suffices to show the following Lemma 14 to prove the openness.

Lemma 14. For any $g \in C_{\frac{1}{2}, 0}^{k-2, \alpha}$ there exists a function $v$ which vanishes at infinity and $v \in C_{\delta}^{k, \alpha}$ for all sufficiently large positive number $\delta$ which solves the Poisson equation with the integrability condition:

$$
\begin{aligned}
\triangle v & =g \\
\int_{X-D} g \omega_{0}^{n} & =0 \\
\int_{B(R)} g \omega_{0}^{n} & =O\left(R^{-\frac{2 n}{n+1}} / 2\right)
\end{aligned}
$$

with estimate

$$
\|v\|_{C_{\delta}^{k, \alpha}} \leq C_{\delta}\|g\|_{C_{\frac{1}{2}, 2, \alpha}^{k-2, \alpha}}
$$

where $C_{\delta}$ is a positive constant depending only on $(X-D, \omega)$ and $\delta$.

Proof. Let $o \in X-D$ be a fixed point and set $\rho(x)=\operatorname{dist}(o, x)$. To show the Lemma 14, we first solve the Dirichlet problem on $B(R)$ 
(the geodesic ball of radius $R$ with center $o$ ):

$$
\begin{aligned}
\triangle v_{R} & =g \\
\left.v_{R}\right|_{B(R)} & =0
\end{aligned}
$$

and then let $R \rightarrow \infty$. If we would apply Moser's iteration technique to (2-86) with the help of the weighted Sobolev inequality (2-22) to derive an a priori $C^{0}$-estimate for $v_{R}$ independent of $R$, the radial function $t^{\frac{1}{n}}$ would become an obstruction, since $\Delta t^{\frac{1}{n}}$ is of exponential decay. This forces us first to estimate Green's function and then use Green's representation for the solution $v_{R}$

$$
v(x)=\int_{B(R)} G_{R}(x, y) g(y) d \mu(y)
$$

where $G_{R}(x, y)$ is the Dirichlet Green's function for the domain $B(R)$. As a function of $y, G_{R}(x, y)$ has a pole at $x$ and positive. Therefore what we want to do is to analyze the behavior of $G_{R}(x, y)$. More precisely, fixing $x$, we divide $X-D$ into two parts $U_{1}$ and $U_{2}$, where $U_{1}$ is a compact part defined by $\rho(z)<\frac{1}{2} \rho(x), U_{2}$ is an asymptotic region defined by $\frac{1}{4} \rho(x)<\rho(z)$. Then we find a constant $a_{R}$ for each $R$ with a growth estimate of the form

$$
\left|a_{R}\right| \leq c(n, \rho(x)) R^{\frac{2}{n+1}}(\log R)(\log \log R)^{2-\frac{1}{n}}
$$

such that $G_{R}(x, y)-a_{R}$, as functions of $y$, converges uniformly on any compact set in $X-D-\{x\}$ (We write $c(n, \rho(x))$ for a positive constant depending only on $n$ and $\rho(x)$ which differs in different occurrences, etc.). To get an effective estimate for $\lim _{R \rightarrow \infty}\left(G_{R}(x, y)-a_{R}\right)$, we first use the weighted Sobolev inequality $(2-22)$ to estimate $a_{R}$ and then we apply Cheng-Yau's local gradient estimate [C-Y1] to conclude that $\lim _{R \rightarrow \infty}\left(G_{R}(x, y)-a_{R}\right)$ grows at most like

$$
\left|\lim _{R \rightarrow \infty}\left(G_{R}(x, y)-a_{R}\right)\right| \leq c(n, \rho(x)) \rho(y)^{\text {const }_{n}}
$$

on $U_{2}$. Since the diameter of $\partial B(R)$ grows like $R^{\frac{1}{n+1}}$, successive use of Cheng-Yau's local gradient estimate and the maximum principle for harmonic functions imply an estimate on the compact part $U_{1}$ :

$$
O s c_{y \in Y_{1}}\left(\lim _{R \rightarrow \infty}\left(G_{R}(x, y)-a_{R}\right)\right) \leq c_{\delta} \rho(x)^{-\delta}
$$

for any positive number $\delta$. 
We begin with deriving estimates for Dirichlet Green's function. Recall that, in [M-S-Y], it is shown that the Sobolev inequality, Harnack inequality and the Euclidean volume growth imply Green's function estimate. Now we have no Sobolev inequality and small volume growth. This time, we use the weighted Sobolev inequality (2-22) and ChengYau's local gradient estimate (see Yau's survey [Y4] for the importance of the gradient estimate).

We follow Aubin's textbook [Au] to construct Dirichlet Green's function on a compact Riemannian manifold with boundary. Let $M^{m}$ be a compact Riemannian manifold of dimension $m$ with boundary. Fixing $P \in M$, we set $r(P, Q)=\operatorname{dist}(P, Q)$. Let $f(x)$ be a smooth nonnegative function on $R$ such that $f(x) \cong 1$ in a neighborhood of 0 and $f(x) \cong 0$ if $x \geq \operatorname{Inj}(P)$, where $\operatorname{Inj}(P)$ is the injectivity radius at $P$. Define

$$
H(r)=f(r) \frac{c_{m}}{r^{m-2}}
$$

where $\frac{c_{m}}{|x-y|^{m-2}}$ is the Green's function of $R^{m}$. Then we have

$$
|\triangle H| \sim K \frac{c_{m}}{r^{m-2}}
$$

in a small neighborhood of $P$ (the size depends on the choice of $f$ only) and

$$
|\triangle H| \sim \frac{c_{m}}{r^{m}}
$$

outside a small neighborhood of $P$. Set

$$
\Gamma_{1}(P, Q)=\triangle_{Q} H(P, Q)
$$

and define $\Gamma_{i}^{\prime} s,(i=1,2, \cdots)$ recursively by setting

$$
\Gamma_{i}(P, Q)=\int_{M} \Gamma_{i-1}(P, R) \Gamma_{1}(R, Q) d \mu_{R} .
$$

Then it is easy to show that all $\Gamma_{i}(P, Q)$ vanishes if $Q \in \partial M$. It follows from the estimates for $\triangle_{Q} H$ that $\Gamma_{i}(P, Q)$ is $C^{1}$-smooth if $i>\frac{m}{2}$. Pick $k>\frac{m}{2}$ and set

$$
G(P, Q)=H(P, Q)+\sum_{i=1}^{k} \Gamma_{i}(P, Q)+F(P, Q)
$$

Then we have

$$
\begin{gathered}
\triangle_{Q} F(P, Q)=\Gamma_{k+1}(P, Q), \\
F(P, Q)=0 \text { if } Q \in \partial M .
\end{gathered}
$$


Suppose $M^{m}=B(R) \subset X-D, P=x$, and $Q=y$. Then we have $G(P, Q)=G_{R}(x, y)$. Set $H(P, Q)=H_{R}(x, y), \Gamma_{i}(P, Q)=\Gamma_{R, i}(x, y)$, $\Gamma=\Gamma_{k+1}$ and $F(P, Q)=F_{R}(x, y)$. Then we can estimate $H_{R}(x, y)$ and $\Gamma_{R, i}(x, y)^{\prime} s$ quite explicitly because of Lemma 10. Set $\rho(z)=\operatorname{dist}(o, z)$ and $\rho(x)=\rho_{0}$, where $o$ is a fixed point. We estimate the maximum of $\left|F_{R}(x, y)\right|$ on $B(R)$ using Moser's iteration technique, where $R>>\rho(x)$. The weighted Sobolev inequality $(2-22)$ and integration by parts imply that for $p>1$

$$
\begin{aligned}
& \left(\int \rho^{-\frac{2 n}{n+1}} \frac{1}{\log \rho}\left|F_{R}\right|^{p \gamma}\right)^{\frac{1}{\gamma}} \\
\leq & \left.\left.C \int \rho^{\frac{2}{n+1}}(\log \rho)(\log \log \rho)^{2-\frac{1}{n}}|d| F_{R}\right|^{\frac{p}{2}}\right|^{2} \\
\leq & \left.\left.C R^{\frac{2}{n+1}}(\log R)(\log \log R)^{2-\frac{1}{n}} \int|d| F_{R}\right|^{\frac{p}{2}}\right|^{2} \\
\leq & C R^{\frac{2}{n+1}}(\log R)(\log \log R)^{2-\frac{1}{n}} \frac{p^{2}}{4(p+1)} \int|\Gamma|\left|F_{R}\right|^{p-1}
\end{aligned}
$$

Fix $1<q<n$ and define $p=p_{-1}$ by $\frac{p-1}{p \gamma}+\frac{1}{q}=1 \Leftrightarrow p=\frac{q(n-1)}{n-q}$. Then we have $p_{-1}>q$. Set $p_{0}=p_{-1} \gamma$. Hölder's inequality implies

$$
\begin{aligned}
& \left(\int \rho^{-\frac{2 n}{n+1}} \frac{1}{\log \rho}\left|F_{R}\right|^{p \gamma}\right)^{\frac{1}{\gamma}} \\
& \leq C R^{\frac{2}{n+1}}(\log R)(\log \log R)^{2-\frac{1}{n}} \frac{p^{2}}{4(p-1)}\left(\int \rho^{-\frac{2 n}{n+1}} \frac{1}{\log \rho}\left|F_{R}\right|^{p \gamma}\right)^{\frac{p-1}{p \gamma}} . \\
& \left(\int\left\{\rho^{\frac{2 n}{n+1}} \log \rho\right\}^{\frac{(p-1) q}{p \gamma}}|\Gamma|^{q}\right)^{\frac{1}{q}} .
\end{aligned}
$$

Thus we have

(2-94) $\left\|\left\{\rho^{-\frac{2 n}{n+1}} \frac{1}{\log \rho}\right\}^{\frac{1}{p_{0}}} F_{R}\right\|_{p_{0}} \leq C R^{\frac{2 n}{n+1}}(\log R)(\log \log R)^{2-\frac{1}{n}} p_{-1}$.

$$
\left\|\left\{\rho^{\frac{2}{n+1}} \log \rho\right\}^{1-\frac{1}{q}} \Gamma\right\|_{q}
$$

where $C$ depends also on the choice of $1<q<n$. Applying Hölder's 
inequality to (2-93), we have for any $p>q$

$$
\begin{aligned}
&\left(\int \rho^{-\frac{2 n}{n+1}} \frac{1}{\log \rho}\left|F_{R}\right|^{p \gamma}\right)^{\frac{1}{\gamma}} \leq \\
& C R^{\frac{2}{n+1}}(\log R)(\log \log R)^{2-\frac{1}{n}} p\left(\int\left\{\rho^{\frac{2 n}{n+1}} \log \rho\right\}^{p-1}|\Gamma|^{p}\right)^{\frac{1}{p}} \\
&\left(\int \rho^{-\frac{2 n}{n+1}} \frac{1}{\log \rho}\left|F_{R}\right|^{p}\right)^{\frac{p-1}{p}}
\end{aligned}
$$

Setting $p=p_{i}=p_{0} \gamma^{i}=p_{-1} \gamma^{i+1}$, we have from (2-95) the following recursive inequality:

$$
\begin{aligned}
& \left\|\left\{\rho^{-\frac{2 n}{n+1}} \frac{1}{\log \rho}\right\}^{\frac{1}{p_{i}+1}} F_{R}\right\|_{p_{i+1}} \leq \\
& \left\{C R^{\frac{2}{n+1}}(\log R)(\log \log R)^{2-\frac{1}{n}} p_{i}\right\}^{\frac{1}{p_{i}}} . \\
& \left\|\left\{\rho^{\frac{2 n}{n+1}} \log \rho\right\}^{\frac{p_{i}-1}{p_{i}}} \Gamma\right\|_{p_{i}}^{\frac{1}{p_{i}}}\left\|\left\{\rho^{-\frac{2 n}{n+1}} \frac{1}{\log \rho}\right\}^{\frac{1}{p_{i}}} F_{R}\right\|_{p_{i}}^{\frac{p_{i}-1}{p_{i}}} .
\end{aligned}
$$

It follows from Lemma 10 that

$$
|\Gamma| \leq c_{n}\left(\rho_{0}^{\frac{n-1}{n+1}}\right)^{2 n}
$$

Iterating the process (2-96) with (2-97) and the initial estimate (2-94) and letting $i \rightarrow \infty$, we have

$$
\begin{aligned}
\left\|F_{R}\right\|_{\infty} & \leq C_{n} \rho_{0}^{\frac{2}{n+1}}\left(\log \rho_{0}\right)\left(\rho_{0}^{\frac{n-1}{n+1}}\right)^{2 n}\left(\frac{1}{\rho_{0}^{\frac{n-1}{n+1}}}\right)^{\frac{2 n}{q} \frac{q}{n}} R^{\frac{2}{n+1}}(\log R)(\log \log R)^{2-\frac{1}{n}} \\
& =c\left(n, \rho_{0}\right) R^{\frac{2}{n+1}}(\log R)(\log \log R)^{2-\frac{1}{n}},
\end{aligned}
$$

where $c\left(n, \rho_{0}\right)=C_{n} \rho_{0}^{\frac{2\left(n^{2}-2 n-2\right)}{n+1}}$.

We choose a positive constant $a_{R}$ such that $F_{R}-a_{R}$ takes its maximum value -1 at a point in $\operatorname{Supp}(\Gamma)$. The estimate $(2-88)$ is now a consequence of (2-98). We now recall Cheng-Yau's local gradient estimate [C-Y1]:

Fact 4 [Theorem 6, C-Y1]. Let $M$ be an n-dimensional complete Riemannian manifold. Let $f$ be a non-negative differentiable function 
defined on a geodesic ball $B(a)$ of radius $a$. Suppose, for some $c>0$, there are constants $m_{1}, m_{2}, \cdots, m_{5}$ such that

$$
\begin{array}{r}
|\nabla(\triangle f)| \leq \frac{1}{2 n} \frac{\sum_{i, j} f_{i j}^{2}}{|\nabla f|}+m_{1} \sqrt{\sum_{i, j} f_{i j}^{2}}+m_{2}|\nabla f|+m_{3}(f+c), \\
\Delta f \leq \frac{1}{4(2 n+1)} \frac{|\nabla f|^{2}}{f+c}+m_{4}|\nabla f|+m_{5}(f+c) .
\end{array}
$$

Then we can find a constant $\alpha_{n}$ depending only on $n$ such that

$$
\frac{|\nabla f(x)|}{f+c} \leq \alpha_{n}\left(\frac{a^{2}}{a^{2}-r^{2}}\right)\left[\max \left\{m_{1}^{2}, m_{2}, m_{3}^{\frac{1}{3}}, m_{4}, m_{5},|K|+\frac{1}{a}\right\}\right]
$$

where $r$ is the distance from $x$ to the center of $B(a)$ and $K$ is the lower bound of the Ricci curvature of $B(a)$.

Now we have $\triangle F=\Gamma$, where $\Gamma$ has compact support around $x$ and can be estimated explicitly. Thus Cheng-Yau's local gradient estimate (2-100) implies that $\left|F_{R}-a_{R}\right|$, as a function of $y$, grows at most like some positive power (depending on $n$ only) of $\rho(x) \rho(y)$. So we have (2-89). Next, we examine the behavior of $F_{R}-a_{R}$ in the compact part $U_{1}$. It follows from Cheng-Yau's gradient estimate that $\left|F_{R}-a_{R}\right|$ is not larger than $\left(\rho_{0} d\right)^{\text {const }_{n}}$ at any point which has distance $d$ from $\operatorname{Supp}(\Gamma)$. Therefore we have

$$
O s c_{S\left(a \rho_{0}\right)}\left(F_{R}-a_{R}\right) \leq \text { const. } \rho_{0}^{\text {const }_{n}}
$$

on a geodesic sphere, where $a$ is a positive number with $a<1$. We write this constant const ${ }_{n}=c_{n}$, say. Since $F_{R}$ is harmonic outside of $\operatorname{Supp}(\Gamma)$, we have from the maximum principle

$$
O s c_{B\left(a \rho_{0}\right)}\left(F_{R}-a_{R}\right) \leq \text { const } . \rho_{0}^{c_{n}}
$$

on a geodesic ball. We now use Cheng-Yau's gradient estimate on the geodesic ball of radius $\alpha \rho_{0}$ (for some $\alpha<1$ ) contained in $B\left(a \rho_{0}\right)$. Since $F_{R}$ is harmonic there, we set $m_{1}=m_{2}=\cdots=m_{5}=0$ in $(2-99)$ and (2-100). Moreover, the Ricci curvature of $\omega_{0}$ decays exponentially. Cheng-Yau's gradient estimate then implies that at the center of the ball

$$
\left|\nabla F_{R}\right| \leq \frac{\text { const }_{n}}{\alpha} \rho_{0}^{c_{n}-1}
$$


Since the metric sphere of radius $\rho_{0}$ has diameter $\rho_{0}^{\frac{1}{n+1}}$, we have for appropriate $\beta<1$

$$
O s c_{B\left(\beta \rho_{0}\right)}\left(F_{R}-a_{R}\right) \leq \operatorname{const}_{n} \rho_{0}^{c_{n}-1+\frac{1}{n+1}} .
$$

Notice that $-1+\frac{1}{n+1}<0$. Iterating this process, we have the series of estimates (2-90). This implies that the Dirichlet Green's function $G_{R}(x, y)(R>>\rho(x))$, as a function of $y$, approaches a constant in the compact region $\rho(y)<\frac{1}{2} \rho(x)$ inverse polynomially of any order with respect to $\rho(x)=\rho_{0}$. The estimates so far obtained for Green's function is already sufficient to derive an a priori decay estimate for $v_{R}$.

$$
\begin{aligned}
v(x)= & \int_{B(R)} G_{R}(x, y) g(y) d \mu(y) \\
= & \int_{B(R)} H(x, y) g(y) d \mu(y)+\int_{B(R)} \sum_{i=1}^{k} \Gamma_{i}(x, y) g(y) d \mu(y) \\
& +\int_{B(R)} F(x, y) g(y) d \mu(y) .
\end{aligned}
$$

Since $g(y)$ is of exponential decay, the first two terms in the extreme right side is of exponential decay with respect to $\rho_{0}$. The third term is written as

$$
\begin{aligned}
& a_{R} \int_{B(R)} g(y) d \mu(y)+\int_{B(R)}\left(F_{R}(x, y)-a_{R}\right) g(y) d \mu(y) \\
& \quad=a_{R} \int_{B(R)} g(y) d \mu(y)+\int_{U_{1}}\left(F-a_{R}\right) g d \mu+\int_{U_{2}}\left(F-a_{R}\right) g d \mu .
\end{aligned}
$$

The integrability condition in (2-83) implies that the first term in the right side is of exponential decay with respect to $R$. Since $g$ is of exponential decay, the third term decays exponentially with respect to $\rho_{0}$. Now the oscillation of $F_{R}(x, y)$ in $y \in U_{1}$ is of order $C_{\delta} \rho_{0}^{-\delta}$ for any positive $\delta$ and $\left|F_{R}(x, y)\right| \leq$ const . $\rho_{0}^{\text {const }_{n}}$. Moreover, the integrability condition implies that the integral of $g$ over $B\left(\rho_{0}\right)$ is of order $\exp \left(-\rho_{0}^{\frac{2 n}{n+1}} / 2\right)$. It then follows that the second term in the right side of (2-102) is of order $C_{\delta} e^{-\delta}$. We thus have a decay estimate for the solution of (2-84). We get decay estimates of derivatives from the interior Schauder estimates, using the coordinates in Lemma 11. 
The estimate (2-98) suggests that $\left|F_{R}(x, y)-a_{R}\right|$ grows like $c\left(n, \rho_{0}\right) \rho^{\frac{2}{n+1}}(\log \rho)(\log \log \rho)$. This is in fact the case, which we show in the following. First we observe

Lemma 15. The radial function $t^{\frac{1}{n}}$ is almost a harmonic function, namely $\Delta t^{\frac{1}{n}}$ is of exponential decay.

Proof.

$$
\sqrt{-1} \partial \bar{\partial} t^{\alpha}=\alpha t^{\alpha-1}\left\{(n \alpha-n)\left(\theta+\frac{\sqrt{-1}}{n t} \partial t \wedge \bar{\partial} t\right)-(n \alpha-n-1) \theta\right\}
$$

implies

$$
\begin{aligned}
\triangle t^{\alpha} & =\left(1+\frac{1}{n}\right)^{-1} \alpha t^{\alpha-1-\frac{1}{n}}\{(n \alpha-n) n-(n-1)(n \alpha-n-1)\}+E \\
& =\left(1+\frac{1}{n}\right)^{-1} \alpha t^{\alpha-1-\frac{1}{n}}(n \alpha-1)+E
\end{aligned}
$$

where $E$ is a nonpositive exponential decay term. Putting $\alpha=\frac{1}{n}$, we get the Lemma.

Lemma 15 and the proof of Lemma 14 imply that there exists a smooth function $w$ which decays polynomially of any order

$$
|w| \leq C_{\delta} t^{-\delta}
$$

such that $F_{0}=t^{\frac{1}{n}}+w$ is harmonic in the complement of a compact set. Since $t^{\frac{1}{n}}$ is a radial function, we can modify $F_{0}$ to be a function such that

$$
\begin{gathered}
F_{0} \equiv \text { const. }>0 \quad \text { if } \rho<\frac{2}{3} \rho_{0} \\
\triangle F_{0} \approx \rho_{0}^{\text {const }}>0 \quad \text { if } y \in U(x)=\left\{\frac{2}{3} \rho_{0}<\rho<\frac{4}{3} \rho_{0}\right\}
\end{gathered}
$$

and $F_{0}$ grows like a radial function $t^{\frac{1}{n}}$. We may assume that $F_{R}(x, y)>$ 0 for $y \in U(x)$ by adding const $_{n} \rho_{0}^{\text {const }_{n}}$ if necessary. From Green's representation formula for the Dirichlet problem on a geodesic ball $B(R)$, we infer

$$
\rho_{0}^{\text {const }}+R^{\frac{2}{n+1}} \leq \inf _{y \in U(x)} F_{R}(x, y) \cdot \rho_{0}^{\text {const }} \operatorname{Vol}(U(x)) .
$$

This and Cheng-Yau's gradient estimate then implies that

$$
\sup _{y \in U(x)} F_{R}(x, y) \leq \text { const } . \rho_{0}^{\text {const }_{n}} R^{\frac{2}{n+1}}
$$


which improves (2-98). Since $F_{0}$ is really a harmonic function which grows like $t^{\frac{1}{n}} \approx \rho^{\frac{2}{n+1}}$, the standard comparison argument (cf. the proof of Theorem 2) implies that the function $\left|F_{R}-a_{R}\right|$ grows like const $_{n} \rho_{0}^{\text {const }_{n}} \rho^{\frac{2}{n+1}}$.

If the "weight" which appears in the right hand side of the weighted Sobolev inequality $(2-22)$ is simply $\rho^{\frac{2}{n+1}}$ instead of $\rho^{\frac{2}{n+1}}(\log \rho)(\log \log \rho)$, then the estimate for $a_{R}$ becomes better:

$$
a_{R} \leq c\left(n, \rho_{0}\right) R^{\frac{2}{n+1}} .
$$

As before, successive use of Cheng-Yau's gradient estimates yield oscillation estimates for $F_{R}$ in the compact region $U_{1}$. Since there exists a function which grows like $\rho^{\frac{2}{n+1}}$ and is harmonic outside of a compact set, the standard comparison argument then implies the best possible estimates for $F_{R}-a_{R}$.

\section{Closedness.}

We first modify the weighted Sobolev inequality $(2-22)$ so that the weight in the right side is $\rho^{\frac{2}{n+1}}$. The weighted isoperimetric inequality is equivalent to the weighted Sobolev inequality

$$
\left(\int \rho^{-\frac{2 n}{n+1}} \frac{1}{\log \rho}|u|^{\frac{2 n}{2 n-1}}\right)^{\frac{2 n-1}{2 n}} \leq C_{w}\left(\omega_{0}\right) \int \rho^{-\frac{n-1}{n+1}}(\log \log \rho)^{1-\frac{1}{2 n}}|d u| .
$$

where $C_{w}\left(\omega_{0}\right)$ is the weighted isoperimetric constant of $\omega_{0}$. Let $w$ be a compactly supported function and set $u=\phi(\rho)|w|^{\frac{2 n-1}{n-1}}$ in the above inequality. From Hölder's inequality, we have

$$
\begin{gathered}
\int \rho^{-\frac{n-1}{n+1}}(\log \log \rho)^{1-\frac{2}{n}}\left|\phi^{\prime}(\rho)\right||w|^{\frac{2 n-1}{n-1}} \\
\leq\left(\int\left(\left|\frac{\phi^{\prime}}{\phi}\right| \rho^{\frac{n}{n+1}}(\log \rho)^{1-\frac{1}{2 n}}(\log \log \rho)^{1-\frac{1}{2 n}}\right)^{2 n}\right)^{\frac{1}{2 n}} \\
\left(\int \rho^{-\frac{2 n}{n+1}} \frac{1}{\log \rho} \phi(\rho)^{\frac{2 n}{2 n-1}}|w|^{\frac{2 n}{n+1}}\right)^{\frac{2 n-1}{2 n}}
\end{gathered}
$$


and

$$
\begin{aligned}
& \int \rho^{-\frac{n-1}{n+1}}(\log \log \rho)^{1-\frac{1}{2 n}} \phi|w|^{\frac{n}{n-1}}|d w| \\
& \leq\left(\int \rho^{-\frac{2 n}{n+1}}(\log \log \rho)^{2-\frac{1}{n}} \phi^{2}|w|^{\frac{2 n}{n-1}}\right)^{\frac{1}{2}}\left(\int \rho^{\frac{2}{n+1}}|d w|^{2}\right)^{\frac{1}{2}} .
\end{aligned}
$$

We put $\phi=\phi(\rho)$ where $\phi=\phi(\rho)$ is a decreasing function that satisfies

$$
\left|\frac{\phi^{\prime}}{\phi}\right| \sim \rho^{-1}(\log N \rho)^{-1-\varepsilon}
$$

with $\varepsilon>0$ and $N>0$. If we choose $\varepsilon>\frac{1}{2 n-2}$ and $N>0$ sufficiently large, we have

$$
\left(\int\left(\left|\frac{\phi^{\prime}}{\phi}\right| \rho^{\frac{n}{n+1}}(\log \rho)^{1-\frac{1}{2 n}}(\log \log \rho)^{1-\frac{1}{2 n}}\right)^{2 n}\right)^{\frac{1}{2 n}}<\frac{C_{w}\left(\omega_{0}\right)}{2}
$$

and

$$
(\log \log \rho)^{2-\frac{1}{n}} \phi^{2}<\frac{1}{\log \rho} \phi^{\frac{2 n}{2 n-1}} .
$$

If we put $u=\phi w$ in (2-101) and apply Hölder's inequality, the estimates (2-102), (2-103), (2-104) and (2-105) imply a weighted Sobolev inequality:

Proposition 13. Let $\left(X-D, \omega_{0}\right)$ be as above. Then there exists a positive decreasing function $\phi=\phi(\rho)$ such that

$$
\left|(\log \phi)^{\prime}\right| \sim \rho^{-1}(\log N \rho)^{-1-\varepsilon}
$$

for some positive numbers $\varepsilon$ and $N$ which fits into the weighted Sobolev inequality

$$
\left(\int \rho^{-\frac{2 n}{n+1}} \frac{1}{\log \rho} \phi^{\frac{2 n}{2 n-1}}|w|^{2 \gamma}\right)^{\frac{1}{\gamma}} \leq C \int \rho^{\frac{2}{n+1}}|d w|^{2}
$$

for any compactly supported $C^{1}$-function $w$, where $C$ is some constant independent of $w$.

We may assume that in (2-106) the weight in the right side is a harmonic function outside a compact set which grows like $\rho^{\frac{2}{n+1}} \approx t^{\frac{1}{n}}$. We use this inequality to derive an a priori $C^{0}$ estimate for the solution 
$u \in C_{\delta}^{k, \alpha}$ ( $\delta$ is sufficiently large) of the Monge-Ampère equation (2-83). We first observe that (2-82) implies

$$
n+\triangle u \geq n\left(\frac{\omega^{n}}{\omega_{0}^{n}}\right)^{\frac{1}{n}}=n e^{-\frac{f}{n}} .
$$

So we have $\Delta u \geq n\left(e^{\frac{-f}{n}}-1\right)$. Arguing as in the proof of openness using Green's representation formula, we get an a priori majorant for $-u$ :

$$
-u \leq C_{\delta} \rho^{-\delta} \text {. }
$$

The majorant of $u$ decays inverse polynomially with sufficiently large degree (as one likes). Therefore we may assume that $u$ is positive provided we replace the exponential decay of $f$ and the integrals in (2-82) by inverse polynomial decay of sufficiently large degree. From here on until obtaining an a priori $C^{0}$ estimate for $u$, we will work on this assumption.

Let $p>1$. Since

$$
\begin{aligned}
& \frac{p^{2}}{4(p-1)}\left(-\sqrt{-1} u|u|^{p-2} \partial \bar{\partial} u\right)= \\
& -\sqrt{-1} \frac{p}{4(p-1)} \partial \bar{\partial}\left(|u|^{p-2} u^{2}\right)+\sqrt{-1} \partial|u|^{\frac{p}{2}} \wedge \bar{\partial}|u|^{\frac{p}{2}}
\end{aligned}
$$

and

$$
\begin{aligned}
\left(1-e^{-f}\right) \omega_{0}^{n} & =\omega_{0}^{n}-\omega^{n} \\
& =-\sqrt{-1} \partial \bar{\partial} u \wedge\left(\omega_{0}^{n-1}+\omega_{0}^{n-2} \omega+\cdots+\omega^{n-1}\right),
\end{aligned}
$$

we have

$$
\begin{aligned}
& \quad \frac{p^{2}}{4(p-1)} \int \rho^{\frac{2}{n+1}}\left(1-e^{-f}\right) u|u|^{p-2} \omega_{0}^{n} \\
& =\int \rho^{\frac{2}{n+1}}\left\{\frac{-p}{4(p-1)} \sqrt{-1} \partial \bar{\partial}\left(|u|^{p-2} u^{2}\right)\right. \\
& \left.\quad \quad+\sqrt{-1} \partial|u|^{\frac{p}{2}} \wedge \bar{\partial}|u|^{\frac{p}{2}}\right\}\left(\omega_{0}^{n-1}+\cdots+\omega^{n-1}\right) \\
& \geq\left.\left.\int \rho^{\frac{2}{n+1}}|d| u\right|^{\frac{p}{2}}\right|^{2} \omega_{0}^{n} \\
& \quad-\frac{p}{4(p-1)} \int \rho^{\frac{2}{n+1}} \sqrt{-1} \partial \bar{\partial}\left(|u|^{p-2} u^{2}\right) \wedge\left(\omega_{0}^{n-1}+\cdots+\omega^{n-1}\right) .
\end{aligned}
$$


Since $u \in C_{\delta}^{k, \alpha}$ for sufficiently large $\delta$, integration by parts gives

$$
\begin{aligned}
& \frac{p}{4(p-1)} \int \rho^{\frac{2}{n+1}} \sqrt{-1} \partial \bar{\partial}\left(|u|^{p-2} u^{2}\right)\left(\omega_{0}^{n-1}+\cdots+\omega^{n-1}\right) \\
& \quad=\frac{p}{4(p-1)} \int\left(\sqrt{-1} \partial \bar{\partial} \rho^{\frac{2}{n+1}}\right)|u|^{p-2} u^{2}\left(\omega_{0}^{n-1}+\cdots+\omega^{n-1}\right)
\end{aligned}
$$

We first consider the case $n=2$. Then we divide the right hand side of (2-109) into two integral:

$$
\frac{p}{4(p-1)} \int|u|^{p-2} u^{2} \sqrt{-1} \partial \bar{\partial} \rho^{\frac{2}{n+1}} \wedge \omega_{0} \leq C \int\left|\triangle \rho^{\frac{2}{n+1}}\right||u|^{p}
$$

where we may consider $\rho^{\frac{2}{n+1}}$ as a harmonic function outside a compact subset, and

$$
\begin{aligned}
& \frac{p}{4(p-1)} \int|u|^{p-1} u \sqrt{-1} \partial \bar{\partial} \rho^{\frac{2}{n+1}} \wedge \sqrt{-1} \partial \bar{\partial} u \\
= & \frac{p^{2}}{(p+1)^{2}(p-1)} \int \sqrt{-1} \partial \bar{\partial} \rho^{\frac{2}{n+1}} \wedge\left\{\frac{p+1}{4 p} \sqrt{-1} \partial \bar{\partial}\left(|u|^{p-1} u\right)\right. \\
\leq & \left.\left.\frac{C}{p} \int \rho^{\frac{2}{n+1}}|d| u\right|^{\frac{p+1}{2}}\right|^{2} \rho^{-2} \\
\leq & \left.\left.\frac{C}{p} \int \rho^{\frac{2}{n+1}}|u||d| u\right|^{\frac{p}{2}}\right|^{2} \rho^{-2} .
\end{aligned}
$$

From (2-108), (2-109), (2-110) and (2-111), we have the most fundamental inequality

$$
\begin{aligned}
\left.\left.\int \rho^{\frac{2}{n+1}}|d| u\right|^{\frac{p}{2}}\right|^{2} \leq & C p \int \rho^{\frac{2}{n+1}}\left|1-e^{-f}\right||u|^{p-1} \\
& +C \int\left|\triangle \rho^{\frac{2}{n+1}}\right||u|^{p}+\left.\left.\frac{C}{p} \int \rho^{\frac{2}{n+1}}|u||d| u\right|^{\frac{p}{2}}\right|^{2}
\end{aligned}
$$

Set $\|u\|_{\infty}=A$ and suppose $p$ is large enough so that

$$
\frac{A C}{p}<\frac{1}{2}
$$

Under this assumption, the third integral in the right hand side of (2112 ) is absorbed in the left hand side. Put $B=\left\|\Delta \rho^{\frac{2}{n+1}}\right\|_{\infty}$. Then $B$ is an a priori constant determined by the background metric $\omega_{0}$. 
Write $g(\rho)=\phi(\rho)^{\frac{2 n}{2 n-1}}$ in (2-106). Under (2-113), the weighted Sobolev inequality (2-106) implies

$$
\begin{aligned}
& \left(\int \rho^{-\frac{2 n}{n+1}} \frac{1}{\log \rho} g(\rho)|u|^{p \gamma}\right)^{\frac{1}{\gamma}} \\
& \quad \leq C p \int \rho^{\frac{2}{n+1}}\left(\left|1-e^{-f}\right|+\frac{A B C}{p} \chi\right)|u|^{p-1}
\end{aligned}
$$

where $\chi$ is the characteristic function of $\Delta \rho^{\frac{2}{n+1}}$ (we regard $\rho^{\frac{2}{n+1}}$ as a function that is harmonic outside a compact set and grows like $\rho^{\frac{2}{n+1}}$ ). We choose $p=p_{-1}>1$ so that (2-113) holds and define $q$ by $\frac{p-1}{p \gamma}+\frac{1}{q}=1$. Put $p_{0}=p_{-1} \gamma$. Applying Hölder's inequality to (2-114) gives

$$
\begin{aligned}
& \left\|\left\{\rho^{\frac{-2 n}{n+1}} \frac{1}{\log \rho} g(\rho)\right\}^{\frac{1}{p_{0}}} u\right\|_{p_{0}} \\
\leq & C p_{-1}\left\|\left\{\rho^{\frac{2 n}{n+1}\left(1-\frac{1}{q}+\frac{1}{n}\right)}\left(\frac{\log \rho)}{g(\rho)}\right)^{1-\frac{1}{q}}\right\}\left(\left|1-e^{-f}\right|+\frac{A B C}{p_{-1}} \chi\right)\right\|_{q}
\end{aligned}
$$

where $p_{-1}$ is of order $\|u\|_{\infty}=A$ up to multiplication of a priori constants and so $\frac{A B C}{p_{-1}}$ is of $O(1)$ as $A \rightarrow \infty$. It follows from (2-115) that the left hand side is bounded above by $A$ up to multiplication of a universal constant. Applying Hölder's inequality to (2-114), we have a recursive inequality:

$$
\begin{aligned}
&\left\|\left\{\rho^{\frac{-2 n}{n+1}} \frac{1}{\log \rho} g(\rho)\right\}^{\frac{1}{p_{i}+1}} u\right\|_{p_{i+1}} \leq \\
&\left(C p_{i}\right)^{\frac{1}{p_{i}}} \|\left\{\rho^{\frac{2 n}{n+1}\left(1-\frac{1}{p_{i}}+\frac{1}{n}\right)}\left(\frac{\log \rho}{g(\rho)}\right)^{1-\frac{1}{p_{i}}}\left(\left|1-e^{-f}\right|+\frac{A B C}{p_{i}} \chi\right) \|_{p_{i}}^{\frac{1}{p_{i}}}\right. \\
&\left\|\left\{\rho^{\frac{-2 n}{n+1}} \frac{1}{\log \rho} g(\rho)\right\}^{\frac{1}{p_{i}}} u\right\|_{p_{i}}^{1-\frac{1}{p_{i}}}
\end{aligned}
$$

where $p_{i}=p_{0} \gamma^{i}$. We iterate the process (2-116) with the initial condition $(2-113) \frac{A C}{p_{-1}}<\frac{1}{2}$ and the corresponding (2-115) and let $i \rightarrow \infty$. Then we have

$$
\|u\|_{\infty} \leq K_{1} A^{\alpha_{1}}
$$

where $\alpha_{1}$ is a positive number with $\alpha_{1}<1$ that depends on $A$, and $K_{1}>0$ is a positive constant bounded above by a constant $K_{0}$ depending on $\omega_{0}$ and $f$ only (independent of $A$ ). We then repeat the iteration 
method with the initial condition $\frac{K A^{\alpha} C}{p_{-1}}<\frac{1}{2}$ and the corresponding $(2-115)$. We then have

$$
\|u\|_{\infty} \leq K_{2}\left(K_{1} A^{\alpha_{1}}\right)^{\alpha_{2}}=K_{2} K_{1}^{\alpha_{2}} A^{\alpha_{1} \alpha_{2}}
$$

Iterating this process, we have

$$
\|u\|_{\infty} \leq\left(\prod_{i=1}^{m} K_{i}^{\prod_{j=i+1}^{m} \alpha_{j}}\right) A \prod_{k=1}^{m} \alpha_{k}
$$

Since $K$ and $\alpha$ decrease as $A$ decreases, we let $m \rightarrow \infty$ to get

$$
\|u\|_{\infty} \leq K_{0}^{\sum_{i=1}^{\infty} \alpha_{0}^{i}}=: J
$$

where $\alpha_{0}$ is a universal constant with $\alpha_{0}<1$, so $J$ in the right hand side is a universal constant. Examining the behavior of $K_{i}^{\prime} s$ in the iteration process, we have

$$
\|u\|_{\infty} \leq C(f)
$$

where $C(f)$ depends on $\omega_{0}$ and $f$ only and as $C(\tau f) \rightarrow 0$ as $\tau \rightarrow 0$. We thus get an a priori $C^{0}$ estimate for $u$ in the case $n=2$. Next, we consider the case $n \geq 3$. But if we note that the difference between $n=2$ and $n \geq 3$ lies essentially in the fact that (2-111) should be replaced by

$$
\begin{gathered}
\frac{p}{4(p-1)} \int|u|^{p-1} u \sqrt{-1} \partial \bar{\partial} \rho^{\frac{2}{n+1}} \wedge(\sqrt{-1} \partial \bar{\partial} u)^{k} \wedge \omega_{0}^{n-k-1} \\
\leq\left.\left.\frac{C}{p} \int \rho^{\frac{2}{n+1}}|d| u\right|^{\frac{p+1}{2}}\right|^{2}\left|\nabla^{2} u\right|^{k} \rho^{-2}
\end{gathered}
$$

we can argue in a similar way to get an a priori $C^{0}$ estimate of $u$.

Once we get a $C^{0}$-estimate, arguing just as in [B-K, Lemma 4] using the Chern-Lu formula (see the arguments below Lemma 13), we obtain an $a$ priori constant $C$ independent of $\tau \in[0,1]$ such that

$$
C \omega_{0}<\omega<C^{-1} \omega_{0}
$$

which is an ordinary $C^{2}$-estimate for $u$. From Lemma $10,\left(X-D, \omega_{0}\right)$ has quasi bounded geometry. Applying the Hölder estimates for the second derivatives of a solution of a fully non-linear elliptic PDE [Gil$\mathrm{T}$, Theorem 17.14], we get an ordinary $C^{2, \alpha}$-estimate in terms of the ordinary $C^{2}$-estimate $(2-119)$ for $u$. Suppose that there exists a solution 
to $(2-84)$ for $\tau \in\left[0, \tau_{0}\right) \subset[0,1]$. We can reach such a situation by successive applications of the implicit function theorem (cf. Lemma 14). Differentiating the equation

$$
\Phi(u)=-\tau f
$$

with respect to $\tau$, we have

$$
\triangle_{\omega} \frac{\partial u}{\partial \tau}=-f
$$

where $\omega=\omega_{0}+\sqrt{-1} \partial \bar{\partial} u_{t}$.

Since we have an ordinary $C^{2, \alpha}$-estimate for $u$, we have the weighted Sobolev inequality (2-106) for $\omega$ with the same constant as that for $\omega_{0}$. We also have $C^{0, \alpha}$-estimate for the coefficients of $\triangle_{\omega}$. Applying Lemma 14 (decay estimates) to (2-120), we have decay estimates

$$
\left|\frac{\partial u}{\partial \tau}\right| \leq C_{\delta} \rho^{-\delta}
$$

for any positive $\delta$. Integrating (2-121) with respect to $\tau$ with the initial condition $u(\tau=0) \equiv 0$, we obtain a decay estimate (independent of $\tau \in[0,1])$ for $u$, which implies that $u$ is of inverse polynomial decay of any order:

$$
|u| \leq C_{\delta} \rho^{-\delta}
$$

independent of $\tau$. We then apply [Gil-T, Theorem 17-15] as in the proof of Theorem 2 to get a desired a priori estimate

$$
\|u\|_{C_{\delta}^{k, \alpha}} \leq C_{\delta}\|f\|_{C_{\frac{1}{2}, 0}^{k-2, \alpha}}
$$

Theorem 6. Let $X$ be a Fano manifold and $D$ a smooth hypersurface such that $c_{1}(X)=[D]$. Then the Monge-Ampère equation (2-82) with $f \in C_{\frac{1}{2}, 0}^{k-2, \alpha}(k \geq 5)$ has a solution $u \in C_{\delta}^{k, \alpha}$ for any positive number $\delta$.

Theorem 7. Let $X$ be a Fano manifold and $D$ a smooth hypersurface such that $c_{1}(X)=[D]$. Then there exists a complete Ricci-flat Kähler metric $\omega=\omega_{0}+\sqrt{-1} \partial \bar{\partial} u$ on $X-D$ where $u \in C_{\delta}^{k, \alpha}$ for any admissible $(k, \alpha)$ with $k \geq 5$ and any positive $\delta$. 
Example 1. Let $(X, D)$ be a pair of a cubic surface $X$ with at worst simple singularities and a smooth hyperplane section $D \subset \operatorname{Reg}(X)$. Then $X-D$ admits a complete Ricci-flat Kähler orbifold-metric.

Example 2. Let $(X, D)$ be a pair of a del-Pezzo surface $X$ with at worst simple singularities and $D$ a smooth curve in $\operatorname{Reg}(X)$ with $c_{1}(X)=[D]$. From the asymptotic behavior of $\omega_{0}$ and a priori estimates for $u$, we have

$$
\frac{1}{8 \pi^{2}} \int_{X-D}\|R\|^{2}=e(X-D)-\sum_{p \in \operatorname{Sing}(X)}\left(e\left(E_{p}\right)-\frac{1}{\left|G_{p}\right|}\right)
$$

where $R$ is the Riemann curvature tensor of $\widetilde{\omega}, E_{p}$ (resp. $G_{p}$ ) is the exceptional set of the minimal resolution (resp. the local fundamental group) of $p$ and $e(\cdot)$ stands for the Euler number. In particular, (2-124) gives an upper bound for the number of simple singularities in $X-D$.

Example 3. Let $(X, D)$ be a pair of a compact complex surface $X$ with at worst simple singularities and a cycle $D$ of rational curves which supports an ample divisor. Assume that $c_{1}(X)=[D]$. For instance, $X=P_{2}(C)$ and $D=$ three lines or $D=$ the nodal cubic curve. Then we can modify the proofs of Theorem 1 and Theorem 7 to show that there exists a complete Ricci-flat Kähler metric on $X-D$ with quadratic volume growth. See [K3].

In the following examples, we propose a geometric picture of the degeneration of Kähler-Einstein K3 surfaces. Although the mathematical footing is still vague in some points, we believe that our existence theorem (Theorem 7) gives the first step toward understanding the singular perturbation of Kähler-Einstein metrics when K3 surfaces degenerate. We start with Friedman's point of view, namely, seeking the geometric objects corresponding to the toroidal compactifications of the coarse moduli space of polarized algebraic K3 surfaces (see [A-M-R-T], [B-B], [Fr] and [Gri]).

Example 4. Let $\mathcal{X} \rightarrow \Delta$ be a type-II degeneration of polarized K3 surfaces such that the singular fiber over 0 consists of $X \cup Y$ glued along a smooth elliptic curve $C$, which is an anti-canonical divisor in each (see $[\mathrm{Ku}],[\mathrm{P}-\mathrm{P}]$ and $[\mathrm{Fr}])$. An example arises in the following way. Consider a family of sextic curves in $P_{2}(C)$ and suppose that $D_{t}$ is smooth for $t \neq 0$ and $D_{0}$ is a smooth cubic curve of multiplicity two. Set $\mathcal{X}^{\prime}=\cup_{t \in \Delta} X_{t}^{\prime}$, where $X_{t}^{\prime}$ is the double plane branched exactly along $D_{t}$. Note that generic $X_{t}^{\prime}$ is a smooth K3 surface with a polarization of degree 2 (the 
lift of the line in $P_{2}(C)$ ). The singular fiber $X_{0}^{\prime}=X^{\prime} \cup Y^{\prime}$ consists of two $P_{2}(C)$ 's glued along a smooth cubic curve $D_{0}$. Note that $\mathcal{X}^{\prime}$ has generically eighteen ordinary double points (i.e., $A_{1}$ singularities) on the double curve in $X_{0}$. We take small resolutions over these 18 points to get a smooth family $\mathcal{X}=\cup_{t \in \Delta} X_{t} \rightarrow \Delta$. Two possibilities in the choice of small resolutions corresponds to blowing up these points in $X^{\prime}$ or $Y^{\prime}$. According to Friedman (see [Gri, p.205-206] and [Fr, Section 5]), there are essentially four possibilities for extending the polarization $h$ of degree 2 on $X_{0}=X \cup Y$. We first look at those (-1)-curves $C$ among eighteen with $h \cdot C>0$. Only those have a meaning from the polarization point of view. It will turns out that those $(-1)$-curves with $h \cdot C=0$ correspond to the Taub-NUT instantons bubbling-off at infinity. The four possibilities arise as follows (see [Gri, p.205, Fig.3]:

(i) Take $X=Y=P_{2}(C)$ and $D$, a smooth cubic curve, as a double curve, and $h=$ a line in each component.

(ii) Take two copies of $P_{2}(C)$ and a smooth cubic curve $D$ as the double curve. In each component take cubic curves passing through 8 points on $D$ with coincident ninth point and blow up resulting 16 intersection points on $D$ in their appropriate components. Let $X$ and $Y$ be $P_{2}(C)$ with their 8 points blown up. The general member of $h$ is the sum of the proper transforms of the cubics with 8 base conditions in each components (with self-intersection number $9-8=1$ ).

(iii) Start as in (ii). Take cubic curves in one component passing through 7 points on $D$ and lines in the other passing through one point. Suppose the residual two intersection points for each curve are coincident. Blow up distinct 8 intersection points in their appropriate components. Let $X$ (resp. $Y$ ) be $P_{2}(C)$ with 7 points (resp. one point) blown up. The general member of $h$ consists of a smooth elliptic curve in $X$ with self-intersection number 2 (the proper transform of a cubic with 7 base conditions) and a rational curve in $Y$ with self-intersection number 0 (the proper transform of a line with one base condition) intersecting on $D$ at the residual 2 points.

(iv) Start as in (ii). Take conics in one component passing through 2 points and twice the linear system of lines in the other passing through one point. Suppose that each curve intersects $D$ with coincident another 4 points. Blow up these 3 base points in their appropriate components and let $X$ (resp. $Y$ ) be $P_{2}(C)$ with 2 points (resp. one point) blown up. Then the general member of $h$ consists of a rational curve in $X$ with selfintersection number 2 and two rational curves in $Y$ with self-intersection number 0 intersecting at the residual 4 points.

We now apply Theorem 7 to propose a picture reconstructing Kähler- 
Einstein K3 surfaces from degenerations:

Cases (i) and (ii): Let $g_{t}$ be the Ricci-flat Kähler metrics on polarized K3 surfaces $X_{t}(t \neq 0)$ with degree 2 . There is a scale change $g_{t} \rightarrow a_{t} g_{t}$ such that $a_{t} g_{t}$ converge to the "union at infinity" of two complete Ricciflat Kähler manifolds $X-D$ and $Y-D$. We can capture the defect $24-e(X)-e(Y)$ of the Euler numbers (which is the defect in the limit of the curvature integra $\frac{1}{8 \pi^{2}} \int\|R\|^{2}$ ) "generically" as the curvature integra for Taub-NUT instantons with concentrated curvature "bubbling off" to infinity. These Taub-NUT instantons corresponds to those $(-1)$-curves $C$ with $h \cdot C=0$, i.e., all the eighteen (-1)-curves. Two complete Ricciflat Kähler surfaces $X-D$ and $Y-D$ may be glued almost trivially (i.e., cylindrically) at infinity. But since $D_{0}$ has self-intersection number 9 in $P_{2}(C)$, there arise 18 points around which two solid tori should be glued to form $S^{3}$. The metric induced on this $S^{3}$ is approximately a Berger sphere metric with very short Hopf fibers and so we fill this $S^{3}$ with a metric ball in the Taub-NUT metric with highly concentrated curvature. Cases (iii) and (iv): In these cases (rescaled) polarized Kähler-Einstein $\mathrm{K} 3$ surfaces converges to a single complete Ricci-flat Kähler manifold. In both cases, this noncompact manifold is the complement of the singular locus $\bar{D}\left(\cong P_{1}(C)\right)$ of a non-normal surface $\bar{X}$ obtained by contracting all curves $C$ in $Y$ with $h \cdot C=0$. This contraction gives rise to a 2:1 holomorphic map of $D$ on the $(-1)$-curve in $Y$. So there are 4 pinch points of $\bar{X}$ which lie on $\bar{D}$. We may reconstruct Kähler-Einstein K3 surfaces from the complete Ricci-flat Kähler metric in Theorem 7 on $X-D=\bar{X}-\bar{D}$ (with Euler number 10 (resp. 5)), 10 (resp. 15) TaubNUT instantons corresponding to the $(-1)$-curves $C$ in $X \cup Y$ with $h \cdot C=0$ and 4 more Taub-NUT instantons corresponding to the 4 pinch points in the case (iii) (resp. (iv)).

The points here are Theorem 7 and the fact that the (rescaled) Berger sphere metrics are realized in the asymptotic region of the TaubNUT metric.

Example 5. For type-III degeneration of polarized K3 surfaces, we can probably show the similar convergence (after suitable scaling) of Ricci-flat Kähler metrics on $X_{t}(t \neq 0)$ to the union at infinity of those complete Ricci-flat Kähler surfaces stated in Example 3 glued together by instantons in Example 3.

In both Examples 4 and 5, we may probably reconstruct an approximately Ricci-flat Kähler metric $\omega_{t}$ on a nearby fiber $X_{t}$ from several number of pieces of Ricci-flat spaces, i.e., complete Ricci-flat Kähler surfaces corresponding to the irreducible components of the singular fiber, 
and the Taub-NUT instantons or "quadratic volume growth instantons" "connecting" these pieces "at infinity". Now Theorem 7 guarantees that the approximation is sufficiently good, i.e., errors are confined in the infinity, so that we can estimate the perturbation $\omega_{t} \rightarrow \widetilde{\omega}_{t}=\omega_{t}+\sqrt{-1} \partial \bar{\partial} u_{t}$ needed to reach a true Ricci-flat metric $\widetilde{\omega}_{t}$.

The author would like to come back to this problem in the near future.

\section{References}

[A-M-R-T] A. Ash, D. Mumford, M. Rapoport and Y. Tai, "Smooth compactification of locally symmetric varieties", Math. Sci. Press, Brooklin MA, 1975.

[A-H] M. Atiyah and N. Hitchin, "The Geometry and Dynamics of Magnetic Monopoles", Princeton University Press, Princeton, New Jersey, 1988.

[Au $\quad$ T. Aubin, "Nonlinear analysis on manifolds. Monge-Ampère equations", Springer-Verlag, New York, 1982.

[BB] W. Baily and A. Borel, Compactification of arithmetic quotients of bounded symmetric domains, Ann. Math., 84 (1966), 422-528.

[B] S. Bando, Bublling out of Einstein metrics, preprint. (Tohoku Math. J., to appear.)

[B-K-N] S. Bando, A. Kasue and H. Nakajima, On a construction of coordinates at infinity on manifolds with fast curvature decay and maximal volume growth, preprint (1988).

[B-K] S. Bando and R. Kobayashi, Ricci-flat Kähler metrics on affine algebraic manifolds, in "Geometry and Analysis on Manifolds (ed. T. Sunada), Proceedings, Katata-Kyoto, 1987", Lecture Notes in Math. 1339, Springer-Verlag, New York/Berlin/ Heidelberg, 1988, pp. 20-31; ibid. II, preprint (1988).

[Bes1] A. Besse, "Géométrie riemannienne en dimension 4", Cedic/ Fernand Nathan, Paris, 1981.

[Bes2] _ "Einstein Manifolds", Springer-Verlag, New York, 1987.

[Bou] J. P. Bourguignon et al, Première Classe de Chern et Courbure de Ricci : Preuve de la Conjecture de Calabi, Astérisque, 58 (1978), Soc. Math. France.

[Ca1] E. Calabi, Métriques kählériennes et fibrés holomorphes, Ann. Sci. Ec. Norm. Sup. Paris, 12 (1979), 269-294.

[Ca2] Isometric families of Kähler structures, in "The Chern Symposium 1979", W. Y. Hsiang et al. (ed.), Springer-Verlag, New York, 1980, pp. 23-39.

[Ch1] S. S. Chern, Characteristic classes of Hermitian manifolds, Ann. of Math., 47 (1946), 85-121. 
[Ch2]

On holomorphic mappings of Hermitian manifolds, in "Proc. Symp. Pure Math. 11", Amer. Math. Soc., Providence, R.I., 1968, pp. 157-170.

[C-Y1] S. Y. Cheng and S. T. Yau, Differential equations on Riemannian manifolds and their geometric applications, Comm. Pure Appl Math., 28 (1975), 333-354.

[C-Y2] On the existence of a complete Kähler-Einstein metric on noncompact complex manifolds and regularity of Fefferman's equation, Comm. Pure appl. Math., 32 (1980), 507-544.

[Cr] C. Croke, Some isoperimetric inequalities and eigenvalue estimates, Ann. Sci. Ec. Norm. Sup., 13 (1980), 419-435.

[F-F] H. Federer and W. Fleming, Normal and integral currents, Ann. of Math, 72 (1960), 458-520.

[Fr] R. Friedman, A new proof of the global Torelli theorem for K3 surfaces, Ann. Math., 120 (1984), 237-269.

[Fr-M] R. Friedman and D. Morrison (ed.), "Birational Geometry of Degenerations", Progress of Mathemtics, vol 29, Birkhäuser, Basel, 1983.

[Fu] K. Fukaya, Hausdorff convergence of Riemannian manifolds and its applications, in this volume.

[Ga] S. Galot, Sobolev inequalities and some geometric applications, in "Spectra of Riemannian Manifolds", Kaigai publishers, Tokyo, 1983.

[Gil-T] D. Gilbarg and N. Trudinger, "Elliptic Partial Differential Equations of Second Order, 2nd edition", Springer-Verlag, New York, 1983.

[Gib] G. Gibbons, Gravitational instantons: a survey, in "Mathematical Problems in Theoretical Physics", K. Osterwalder (ed.) Lecture notes in Physics, No.116, Springer-Verlag, 1979.

[Gib-Haw] G. Gibbons and S. Hawking, Gravitational multi-instantons, Phys. Lett, 78B (1978), 430-432.

[G-P] G. Gibbons and C. Pope, The positive action conjecture and asymptotically Euclidean metrics in quantum gravity, Comm. Math. Phys., 66 (1979), 267-290.

[G-P-R] G. Gibbons, C. Pope and H. Römer, Index theorem boundary terms for gravitational instantons, Nuclear Phys., B157 (1979), 377-386.

[Gri] P. Griffiths (ed.), "Topics in Transcendental Algebraic Geometry", Annals of mathematics Studies Number 106, 1984.

[Gri-Har] P. Griffiths and J. Harris, "Principles of Algebraic Geometry", John Willy and Sons, New York, 1978.

[Haw] S. Hawking, Gravitational instantons, Phys. Lett, 60A (1977), 81-83.

[Hir] F. Hirzebruch, "Topological Methods in Algebraic Geometry", Springer-Verlag, New York, 1962. 
[H-K] F. Hirzebruch and K. Kodaira, On the complex projective space, J. Math. Pure Appl., 36 (1957), 201-216.

[Hit1] N. Hitchin, Polygons and gravitons, Math. Proc. Camb. Phil. Soc., 85 (1979), 465-476.

[Hit2] - Twistor construction of Einstein metrics, in "Global Riemannian Geometry", N. Hitchin and T. Willmore (eds), Ellis Horwood, Chichester, 1984.

[H-K-L-R] N. Hitchin, A. Karlehede, U. Lindstrom and M. Rocek, Hyperkähler metrics and supersymmetry, Comm. Math. Phys., 108 (1987), 535-589.

[I] M. Itoh, Quaternion structure on the moduli space of Yang-Mills connections, Math. Ann., 276 (1987), 581-593.

[K1] R. Kobayashi, Moduli of Einstein metrics on a K3 surface and degeneration of type I, in this volume.

[K2] Ricci-flat Kähler metrics on the complement of an anticanonical divisor of Fano manifolds, preprint, 1989.

[K3] W Weighted Sobolev inequalities and Ricci-flat Kähler surfaces with quadratic volume growth, in preparation.

[K-N-S $]$ R. Kobayashi, S. Nakamura and F. Sakai, A numerical characterization of ball quotients for normal surfaces with branch lici, in this volume.

[K-T] R. Kobayashi and A. Todorov, Polarized period map for generalized K3 surfaces and the moduli of Einstein metrics, Tohoku Math. J., 39 (1987), 341-363.

[Ko1] S. Kobayashi, On compact Kähler manifolds with positive definite Ricci tensor, Ann. of Math., 7 (1961), 570-574.

[Ko2] _ "Differential Geometry of Holomorphic Vector Bundles", Iwanami Shoten Publishers / Princeton University Press, Tokyo / New York, 1987.

[K-N] S. Kobayashi and K. Nomizu, "Foundations of Differential Geometry, I,II", John willy and Sons, New York, 1969.

[K-O] S. Kobayashi and T. Ochiai, On complex manifolds with positive tangent bundles, J. Math. Soc. Japan, 22 (1970), 499-525; Characterization of complex projective space and hyperquadric, J. Math. Kyoto Univ., 13 (1972), 31-47.

[Kr1] P. Kronheimer, Monopoles and Taub-NUT metrics, preprint (1985).

[Kr2] P. Kronheimer, ALE gravitational instantons, Thesis, Oxford Univ. (1986); Instantons gravitationals et singularité de Klein, C. R. Acad. Sc. Paris, 303 (1986), 53-55.

[Ku] V. Kulikov, Degenerations of K3 surfaces and Enriques surfaces, Math. USSR Izvestiya, 11 (1977), 957-989.

[L] C. LeBrun, Complete Ricci-flat Kähler metrics on $C^{n}$ need not be flat, preprint (1989). 
[Li] P. Li, On the Sobolev constant and the p-spectrum of a compact Riemannian manifold, Ann. Sci. Ec. Norm. Sup., 13 (1980), 451-469.

[Lu] V. C. Lu, Holomorphic mappings of complex manifolds, J. Differential Geometry, 2 (1968), 299-312.

[M-S] J. Milnor and J. Stasheff, "Characteristic Classes", Ann. of Math. Study 76, Princeton University Press, Princeton, New Jersey, 1974.

[Mok] N. Mok, An embedding theorem of complete Kähler manifolds of positive Ricci curvature into quasi-projective varieties, to appear.

[M-S-Y] N. Mok, Y.T. Siu and S.T. Yau, The Poincaré-Lelong equation on complete Kähler manifolds, Compositio Math, 44 (1981), 183-218.

[M] J. Moser, On Harnack's Theorem for Elliptic Differential Equations, Comm. Pure Appl. Math., 14 (1961), 577-591.

[N] A. Nadel, Multiplier ideal sheaves and Kähler-Einstein metrics of positive scalar curvature, preprint, The Institute for Advanced Study.

[Pa1] D. Page, A physical picture of the K3 gravitational instanton, Phys. Lett., 80B (1978), 55-57.

[Pa2] - A periodic but nonstationary gravitational instanton, Phys. Lett. B, 100 (1981), 313-315.

[Pe] M. Perry, Gravitational instantons, in "Seminar on Differential Geometry", S. T. Yau (èd.) Ann. Math. Studies 102, Princeton Univ. Press, Princeton, 1982, pp. 602-630.

[P-P] U. Persson and H. Pinkham, Degenerations of surfaces with trivial canonical divisor, Ann. Math., 113 (1981), 45-66.

[Sa] I. Satake, On compactifications of the quotient spaces for arithmetically defined discontinuous groups, Ann. Math., 72 (1960), 555-580.

[Si1] Y. T. Siu, The existence of Kähler-Einstein metrics on manifolds with positive anticanonical line bundle and finite symmetry group, Ann. Math., 127 (1988), 147-162.

[Si2] _ "Lectures on Hermitian-Einstein Metrics and Stable Bundles and Kähler-Einstein Metrics", Birkhäuser, Basel, 1987.

[T1] G. Tian, On Kähler-Einstein metrics on certain Kähler manifolds with $c_{1}(M)>0$, Invent. Math., 89 (1987), 225-246.

[T2] On Calabi's conjecture for complex surfaces with positive first Chern class, preprint, Institute for Advanced Study,.

[T-Y] G. Tian and S. T. Yau, Kähler-Einstein metrics on complex manifolds with $c_{1}>0$, Comm. Math. Phys., 112 (1987), 175-203.

[Y1] S. T. Yau, Harmonic functions on complete Riemannian manifolds, Comm. Pure Appl. Math., 28 (1975), 201-228. 
[Y2] - On the Ricci curvature of a compact Kähler manifold and complex Monge-Ampère equation,I, Comm. Pure Appl. Math., 31 (1978), 339-411.

[Y3] _ A general Schwarz lemma for Kähler manifolds, Amer. J. Math., 100 (1978), 197-203.

[Y4] Survey on partial differential equations in differential geometry, in "Seminar on Differential Geometry", Annals of Mathematics Studies 102, Princeton University Press, Princeton, New Jersey, 1982, pp. 3-71.

[Y5] W Nonlinear analysis in geometry, L'Enseig. Math., 33 (1987), 109-158.

[Ye] S. K. Yeung, Complete Kähler manifolds of positive Ricci curvature, Math. Z., 204 (1990), 187-208.

Department of Mathematics

College of Arts and Sciences

University of Tokyo

Komaba, Tokyo 153

Japan 